B O N N E V I L L E Monitoring the Migrations of Wild Snake River Spring/Summer Chinook Salmon Juveniles

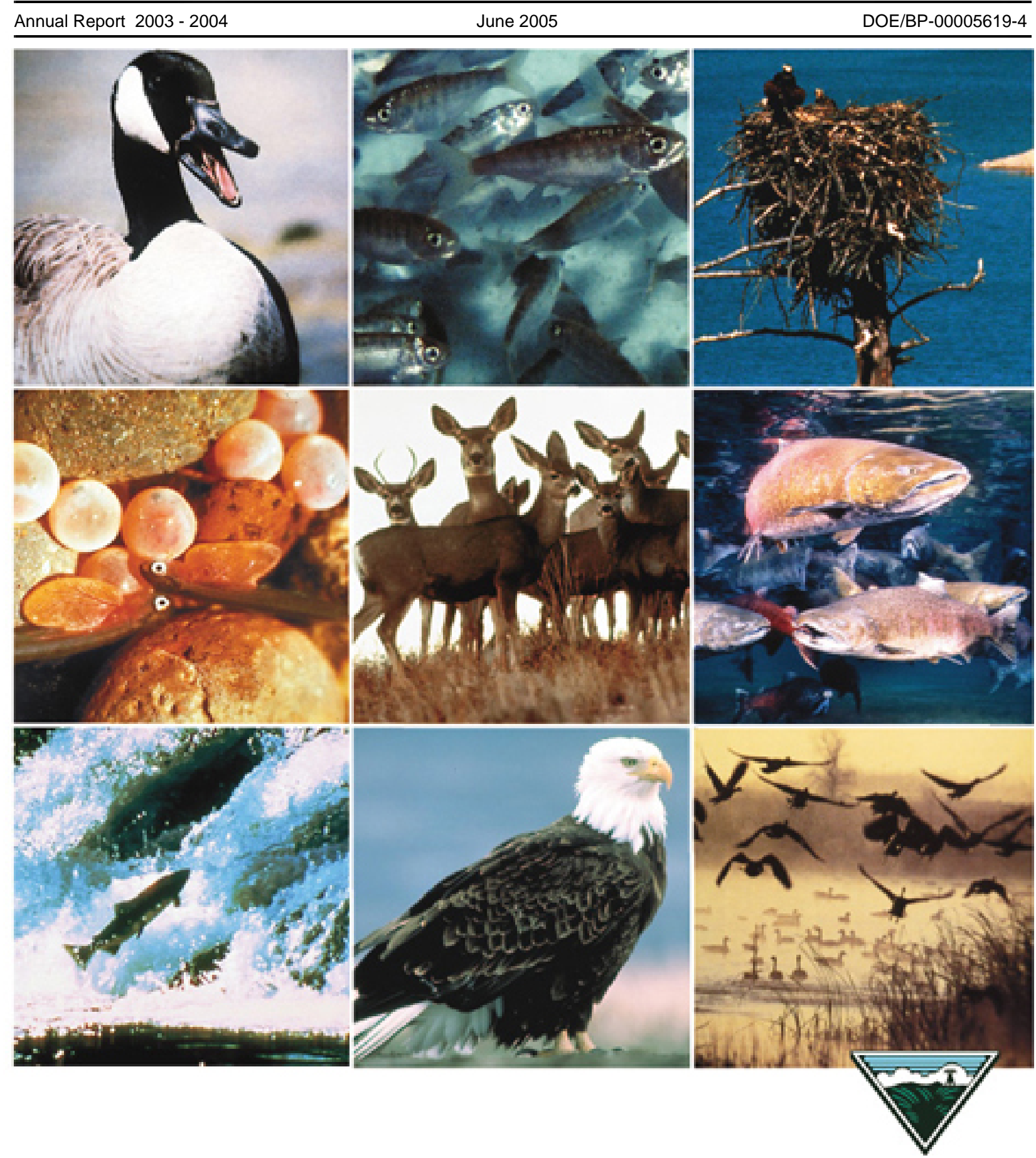


This Document should be cited as follows:

Achord, Stephen, Jacob Hodge, Benjamin Sandford, Eric Hockersmith, Kenneth McIntyre, Neil Paasch, John Williams, "Monitoring the Migrations of Wild Snake River Spring/Summer Chinook Salmon Juveniles", 2003-2004 Annual Report, Project No. 199102800, 102 electronic pages, (BPA Report DOE/BP-00005619-4)

\author{
Bonneville Power Administration \\ P.O. Box 3621 \\ Portland, OR 97208
}

This report was funded by the Bonneville Power Administration (BPA), U.S. Department of Energy, as part of BPA's program to protect, mitigate, and enhance fish and wildlife affected by the development and operation of hydroelectric facilities on the Columbia River and its tributaries. The views in this report are the author's and do not necessarily represent the views of BPA. 


\title{
Monitoring the Migrations of Wild Snake River Spring/Summer Chinook Salmon Juveniles, 2004
}

Stephen Achord, Jacob M. Hodge, Benjamin P. Sandford, Eric E. Hockersmith, Kenneth W. McIntyre, Neil N. Paasch, and John G. Williams

\author{
Report of research by \\ Fish Ecology Division \\ Northwest Fisheries Science Center \\ National Marine Fisheries Service \\ National Oceanic and Atmospheric Administration \\ 2725 Montlake Boulevard East \\ Seattle, Washington 98112-2097 \\ for \\ U.S. Department of Energy \\ Bonneville Power Administration \\ Division of Fish and Wildlife \\ P.O. Box 3621 \\ Portland, Oregon 97208-3621 \\ Project 1991-028-00 \\ Contract No. 00005619
}

June 2005 


\section{EXECUTIVE SUMMARY}

This report provides the 2003-2004 results from an ongoing project to monitor the behavior of wild spring/summer Chinook salmon juveniles in the Snake River Basin. We report estimated parr-to-smolt survival and arrival timing at Lower Granite Dam of wild fish PIT-tagged tagged in Idaho by the National Marine Fisheries Service (NMFS). We also report arrival timing at the dam of fish tagged in Oregon streams by the Oregon Department of Fish and Wildlife (ODFW).

Principal results from our tagging and interrogation activities during 2003-2004 are listed below:

1) In July and August 2003, we PIT tagged and released 18,346 wild Chinook salmon parr in 16 Idaho streams.

2) Average overall observed mortality from collection, handling, tagging, and after a 24-hour holding period was $1.1 \%$.

3) Of the 2,498 Chinook salmon parr PIT tagged and released in Valley Creek in summer 2003, 10.0\% (251) were detected at two in-stream PIT-tag monitoring systems in lower Valley Creek from late summer 2003 to spring 2004. Of these, $59 \%$ were detected in late summer/fall, $32.3 \%$ in winter, and $8.8 \%$ in spring. The estimated parr-to-smolt survival to Lower Granite Dam for each of these groups was $6.6 \%$ for the late-summer/fall group, $16.9 \%$ for the winter group, and $43.9 \%$ for the spring group. An estimated 28 to $40 \%$ of all summer-tagged parr survived to move out of Valley Creek, and their estimated survival from that point to the dam was $13.2 \%$. Overall estimated parr-to-smolt survival at the dam for all summer-tagged fish from this stream was 5.5\%. Development and improvement of in-stream PIT-tag monitoring systems continued throughout 2003 and 2004.

4) At Little Goose Dam in 2004, length and weight was measured for 974 recaptured fish from 16 Idaho streams. Fish had grown an average of $41.3 \mathrm{~mm}$ in length and $8.5 \mathrm{~g}$ in weight over an average of 277 days. Their mean condition factor declined from 1.28 at release (parr) to 0.99 at recapture (smolt).

5) Fish that were larger at release were detected at a significantly higher rate the following spring and summer than their smaller cohorts $(P<0.001)$. 
6) Fish that migrated through Lower Granite Dam in April and May were significantly larger at release than fish that migrated after May $(P<0.001)$.

7) In 2004, detections at Lower Granite Dam peaked during low flows of $64.2 \mathrm{kcfs}$ on 15 April for parr tagged during summer 2003 (from 16 streams in Idaho and 4 streams in Oregon). The 10th, 50th, and 90th percentile passage occurred on 16 April, and 3 May, and 26 May, respectively.

8) Estimated parr-to-smolt survival to Lower Granite Dam for Idaho and Oregon streams (combined) averaged 8.1\% (range 4.1-18.0\% depending on stream of origin). This was the lowest average survival rate measured in the last 12 years. It may have related to high parr densities in 2003 that resulted from a comparatively large number of wild spawners in 2002.

In 2004, the 50th and 90th percentile passage dates for wild fish passing Lower Granite Dam occurred in early and late May, respectively. In 2004, we observed close to normal climatic conditions, but flows were considered low during most of the spring. Clearly, complex interrelationships of several factors drive the annual migration timing of these stocks. 


\section{CONTENTS}

EXECUTIVE SUMMARY ….......................................................................... ii

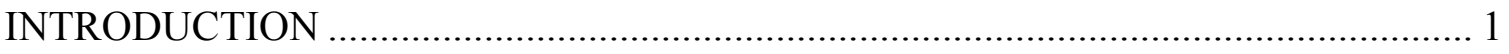

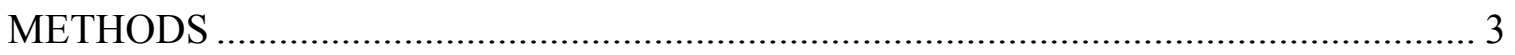

Fish Collection and Tagging ................................................................... 3

Interrogation at In-stream PIT-Tag Monitors ................................................... 3

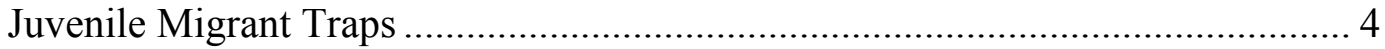

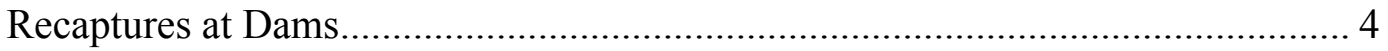

Interrogation at Dams and Lower Columbia River......................................... 5

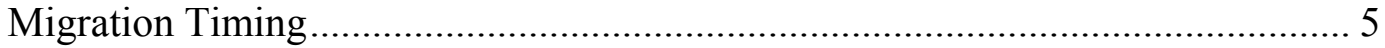

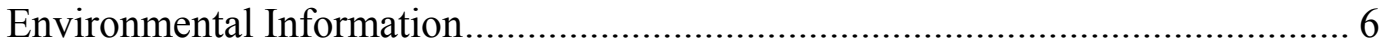

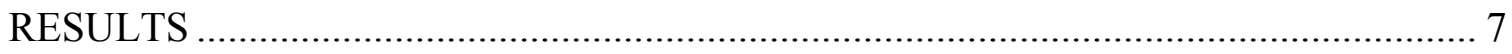

Fish Collection and Tagging .................................................................... 7

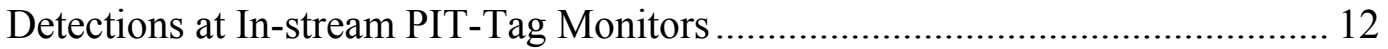

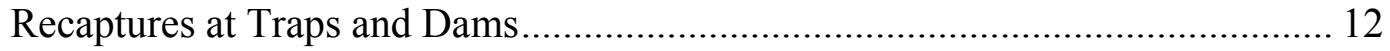

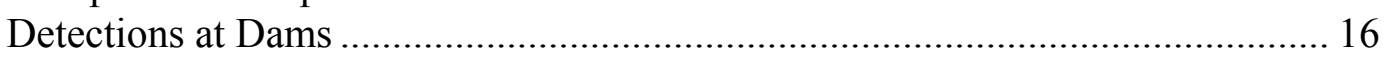

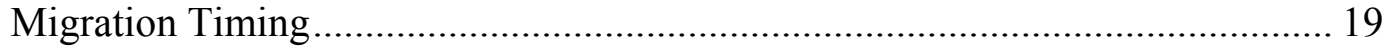

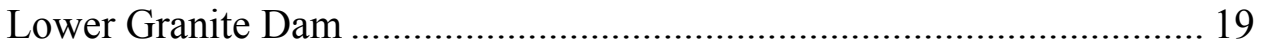

Comparison with Flows ......................................................................... 29

Environmental Information......................................................................... 29

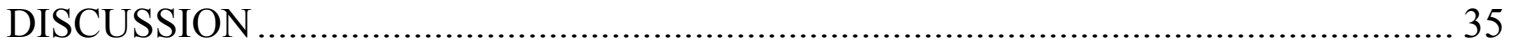

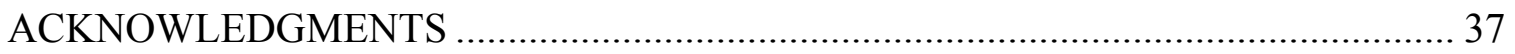

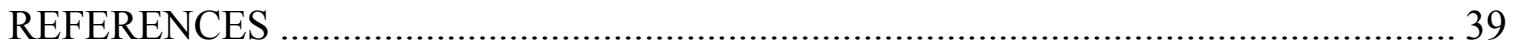

APPENDIX: Data Tables and Figures.................................................................. 44 


\section{INTRODUCTION}

This report provides information on PIT-tagging of wild Chinook salmon parr in Idaho in 2003 and the subsequent monitoring of these fish and similarly tagged fish from Oregon. We report estimated parr-to-smolt survival and arrival timing of these fish at Lower Granite Dam, as well as interrogation data collected at several other sites throughout the Snake and Columbia River system. This research continues studies that began under Bonneville Power Administration (BPA) funding in 1991. Results from previous study years were reported by Achord et al. (1994; 1995a,b; 1996a; 1997; 1998; 2000; 2001a,b; 2002, 2003, 2004). Goals of this ongoing study are:

1) Characterize the migration timing and estimate parr-to-smolt survival of different stocks of wild Snake River spring/summer Chinook salmon smolts at Lower Granite Dam.

2) Determine whether consistent migration patterns are apparent.

3) Determine what environmental factors influence migration patterns.

4) Characterize the migration behavior and estimate survival of different wild juvenile fish stocks as they emigrate from their natal rearing areas.

This study provides critical information for recovery planning, and ultimately recovery for these ESA-listed wild fish stocks.

In 2003-2004, we also continued to measure water temperature, dissolved oxygen, specific conductance, turbidity, water depth, and $\mathrm{pH}$ at five monitoring stations in the Salmon River Basin, Idaho for the Baseline Environmental Monitoring Program. These data, along with parr/smolt migration, survival, and timing data, will help to discern patterns or characteristic relationships between fish movement/survival and environmental factors. 


\section{METHODS}

\section{Fish Collection and Tagging}

Wild Chinook salmon parr in the Grande Ronde and Imnaha River drainages in northeast Oregon were tagged by the Oregon Department of Fish and Wildlife (ODFW) in 2003. All tagging, detection, and timing information for fish from these streams in 2003-2004 will be reported by ODFW. However, in concurrence with ODFW, we report here the arrival timing at Lower Granite Dam of summer-tagged fish from these Oregon streams.

In 2003, we used the same collection and PIT-tagging procedures to mark parr in Idaho streams as we used in previous years (see Matthews et al. 1990; Achord et al. 1994, 1995a,b, 2003, 2004).

\section{Interrogation at In-stream PIT-Tag Monitors}

Until recently, opportunities to monitor migrating PIT-tagged wild juvenile fish were limited to a few in-stream or river traps (these traps required operators and were not passive monitoring sites), juvenile fish bypass systems at collector dams, and a surface pair-trawl detection system operated in the Columbia River estuary. In an effort to detect fish closer to tagging sites, we began development of in-stream PIT-tag monitoring systems in Valley Creek in 2002 (Downing et al. 2004). We placed detector systems at two sites located $1.6 \mathrm{~km}$ apart (VC1 and $\mathrm{VC} 2$ ).

Development of the Valley Creek monitoring systems continued throughout 2003 and 2004; details of this development are described by Achord et al. (2004). In addition to continued improvements to the monitoring electronics, we replaced the antennas at both sites with larger 3.0-m long by $61-\mathrm{cm}$ high (outside dimensions) antennas (constructed of $7.6 \mathrm{~cm}$ PVC pipe). Both systems were set up to automatically interrogate, store, and transmit data to the PIT Tag Information System (PTAGIS), a database operated by the Pacific States Marine Fisheries Commission (PSMFC 1996). Here we report data collected at the Valley Creek sites from August 2003 through July 2004. 


\section{Juvenile Migrant Traps}

Some fish PIT tagged as parr in natal rearing areas are subsequently collected at migrant traps (Figure 1). During fall 2003 and spring 2004, juvenile migrant fish traps were operated at Knox Bridge on the South Fork of the Salmon River, on Lake Creek, near Chinook Campground on the Secesh River, on Marsh Creek, and near the Sawtooth Hatchery on the upper Salmon River. Also during spring 2004, juvenile migrant fish traps were operated on the lower Salmon River near Whitebird, Idaho, and on the Snake River at Lewiston, Idaho. Traps were operated by the Nez Perce Tribe and the Idaho Department of Fish and Game.

Generally, fish at these traps were anesthetized, scanned for PIT tags, and then measured for length and weight. Upon recovery from the anesthetic, all fish were released back to the streams or rivers.

\section{Recaptures at Dams}

While collecting and PIT tagging fish at the dams for various studies, NMFS and other personnel occasionally encounter wild fish that are already PIT tagged. In such cases, biological data are usually collected from these fish. To increase sample sizes for parr-to-smolt growth information on previously PIT-tagged wild fish, in 2004 we continued efforts begun in 2001 to utilize the PIT-tag separation-by-code system (Downing 2001) at Little Goose Dam. The system was programmed to separate up to a maximum of 100 wild fish from each stream so that we could take length and weight measurements from a sample of fish. All fish that were separated at the dam were handled using water-to-water transfers and other best handling practices. After handling, all tagged and untagged fish were returned to the bypass system for release below the dam.

In addition to length and weight measurements on these wild smolts at Little Goose Dam, a Fulton-type condition factor (CF) was calculated as

$$
C F=\frac{\text { weight }(\mathrm{g})}{\operatorname{length}(\mathrm{mm})^{3}} \times 10^{5}
$$

Condition factor was calculated for these fish both at release and recapture. 


\section{Interrogation at Dams and Lower Columbia River}

During spring and summer 2004, Chinook salmon that were PIT tagged as parr in 2003 and survived the winter migrated volitionally downstream through hydroelectric dams on the Snake and Columbia Rivers. Of the eight lower Snake River and Columbia River dams encountered by these fish, the following six were equipped with smolt collection and/or PIT-tag interrogation systems: Lower Granite, Little Goose, and Lower Monumental Dams on the Snake River, and McNary, John Day, and Bonneville Dams on the Columbia River.

At these six dams, all smolts guided from turbine intakes into juvenile bypass systems were electronically monitored for PIT tags. The PIT-tag interrogation systems were the same as those described by Prentice et al. (1990). Dates and times to the nearest second were automatically recorded on a computer as PIT-tagged fish passed each detector. Detection data were transferred once daily to PTAGIS (PSMFC 1996).

PIT-tagged fish were also monitored by a surface pair-trawl detector operated in the upper estuary of the Columbia River (approximately $150 \mathrm{~km}$ downstream from Bonneville Dam). For details of its operation, see Ledgerwood et al. (2004).

\section{Migration Timing}

We monitored within-season migration timing at Lower Granite Dam based on daily detection numbers (of all wild PIT-tagged Chinook salmon smolts) expanded relative to estimated daily detection probabilities. Detection probabilities were calculated using the methods of Sandford and Smith (2002) to provide an estimate of the number of PIT-tagged wild spring/summer Chinook salmon smolts that passed the dam each day. These daily totals were then summed to obtain a yearly survival estimate, which we compared to survival estimates from previous years.

Migration timing at Lower Granite Dam was calculated by totaling the (expanded) number of detections in 3-day intervals and dividing by total detections during the season. This method was applied to detection data for fish from combined streams.

There was no straightforward way of comparing arrival timing distribution at Lower Granite Dam between streams (in terms of the dates of 10th, median, and 90th passage percentiles) to find statistically significant differences. We used an approach analogous to analysis of variance with multiple comparisons. Bootstrap methods were 
used to calculate estimates of the standard error for each statistic (Efron and Tibshirani 1993). A "representative" estimate of variance for each statistic was then calculated as the median of the standard errors (SEs) for fish from all 20 streams. This method assumed that the timing of passage percentiles had similar distributions among streams. The Student-Newmann-Keuls (SNK) multiple comparison method was used to make comparisons between streams for each statistic $(\alpha=0.05$; Petersen 1985).

We also examined the migration timing at Lower Granite Dam of individual populations over a period of years to determine similarities or differences between years and between populations. We chose populations with 7 or more years of timing data for these analyses. Comparisons of the 10th, 50th, and 90th percentile passage dates were made among 18 streams using a two-factor analysis of variance (ANOVA). "Year" was considered a random factor and "stream" a fixed factor. Residuals were visually examined to assess normality. Treatment means were compared using Fisher's least significant difference (Peterson 1985). Statistical significance was set at $\alpha=0.05$.

\section{Environmental Information}

In 2003-2004, we collected hourly measurements of water temperature, dissolved oxygen, specific conductance, turbidity, water depth, and $\mathrm{pH}$ from the following locations: 1) Marsh Creek, 2) Valley Creek, 3) Sawtooth Hatchery in the upper Salmon River, 4) South Fork of the Salmon River (Knox Bridge), and 5) Secesh River (near Chinook Campground). All monitoring systems except the system at Valley Creek were close to juvenile migrant fish traps. The water quality monitor at Valley Creek was located near our in-stream PIT-tag monitoring system (VC2). 


\section{RESULTS}

\section{Fish Collection and Tagging}

From 22 July to 30 August 2003, we collected 25,209 wild Chinook salmon parr in 16 Idaho streams (Figure 1) over a distance of about 35 stream kilometers and an area of approximately $304,519 \mathrm{~m}^{2}$ (Table 1; Appendix Table 1). Of these fish, 18,346 were PIT tagged and released back into the streams along with the remaining non-tagged live fish. Some fish were not tagged because of small size, injury, precocious maturation, or because excess numbers were collected. In addition, some fish were collected for genetic and marine derived nutrient samples. Numbers of tagged fish released per stream ranged from 52 in Rush Creek to 2,498 in Valley Creek (Table 1; Appendix Tables 1 and 2a).

Fork lengths of all collected Chinook salmon parr ranged from 36 to $296 \mathrm{~mm}$ (mean $60.4 \mathrm{~mm}$ ) and weights ranged from 0.4 to $23.5 \mathrm{~g}$ (mean $3.2 \mathrm{~g}$ ). Fork lengths of tagged and released Chinook salmon parr ranged from 43 to $184 \mathrm{~mm}$ (mean $63.2 \mathrm{~mm}$ ) (occasionally fish smaller than $55 \mathrm{~mm}$ are inadvertently tagged) and weights ranged from 1.0 to $11.4 \mathrm{~g}$ (mean $3.4 \mathrm{~g}$; Appendix Table 1). In 2003, collection areas within the streams were further delineated by recording Global Positioning System (GPS) coordinates using Universal Transverse Mercator (UTM) grid (Appendix Table 2b).

Other than Chinook salmon parr, sculpin were the most abundant species observed during collection operations (Table 2). However, the counts of non-target fish were not from systematic sampling for these species and thus do not represent total abundances in the collection areas. The samples targeted only wild Chinook salmon.

Mortality associated with collection and tagging procedures was low, as was 24-h tag loss (only one in Loon Creek). Average collection mortality was $1.0 \%$, average tagging and 24 -hour delayed mortality was $0.1 \%$, and average overall observed mortality was 1.1\% (Table 3; Appendix Table 3). 


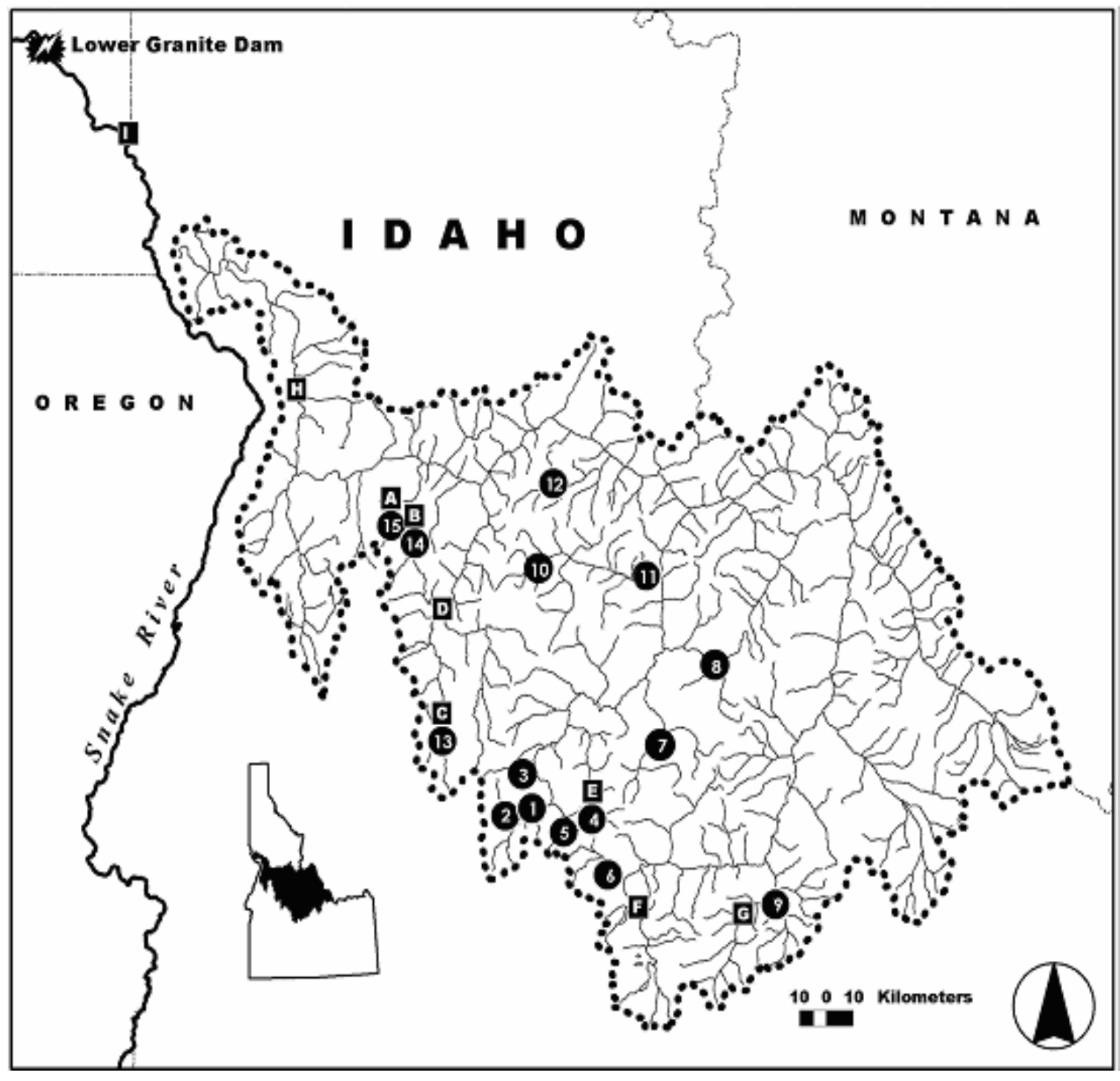

Figure 1. Wild spring/summer Chinook salmon parr were PIT tagged during 2003 in the following streams:

1-Bear Valley Creek

2-Elk Creek

3-Sulphur Creek

4-Marsh Creek

5-Cape Horn Creek
6-Valley Creek

7-Loon Creek

8-Camas Creek

9-Herd Creek

10-Big Creek (upper)
11-Big Creek (lower)/Rush Creek 12-W.F. Chamberlain/Chamberlain $\mathrm{Cr}$ 13-South Fork Salmon River 14-Secesh River 15-Lake Creek

Juvenile migrant fish traps shown above are as follows:

A-Lake Creek Trap

B-Secesh River Trap

C-South Fork Salmon River Trap
D-Lower S.F. Salmon River Trap

E-Marsh Creek Trap

F-Sawtooth Trap
G-East Fork Salmon River Trap

H-Salmon River Trap

I-Snake River Trap 
Table 1. Summary of collection, PIT tagging, and release of wild Chinook salmon parr with average fork lengths and weights, approximate distances, and estimated areas sampled in streams of Idaho during July and August 2003.

\begin{tabular}{|c|c|c|c|c|c|c|c|c|}
\hline & \multicolumn{2}{|c|}{ Number of fish } & \multicolumn{2}{|c|}{ Average length (mm) } & \multicolumn{2}{|c|}{ Average weight (g) } & \multirow{2}{*}{$\begin{array}{l}\text { Collection area to } \\
\text { mouth of stream } \\
(\mathrm{km})\end{array}$} & \multirow{2}{*}{$\begin{array}{c}\text { Estimated area } \\
\text { sampled in } \\
\text { stream }\left(\mathrm{m}^{2}\right)\end{array}$} \\
\hline & Collected & $\begin{array}{c}\text { Tagged \& } \\
\text { released }\end{array}$ & Collected & Tagged & Collected & Tagged & & \\
\hline Bear Valley Creek & 1,995 & 1,494 & 59.1 & 61.4 & 2.8 & 3.0 & $9-9.5 \& 13-15$ & 2,070 \\
\hline Elk Creek & 1,819 & 1,520 & 61.4 & 62.9 & 3.1 & 3.3 & $0-3$ & 20,459 \\
\hline Marsh Creek & 1,812 & 1,535 & 63.3 & 64.6 & 3.5 & 3.7 & $11-14$ & 10,614 \\
\hline Sulphur Creek & 1,319 & 1,048 & 62.1 & 62.9 & 3.0 & 3.1 & $5-5.5$ & 1,400 \\
\hline Cape Horn Creek & 2,319 & 671 & 53.8 & 61.4 & 2.8 & 3.4 & $0-2 \& 5-6$ & 21,075 \\
\hline Valley Creek & 3,152 & 2,498 & 61.1 & 62.8 & 3.4 & 3.5 & $4,6,18$ & 34,989 \\
\hline Loon Creek & 952 & 860 & 63.4 & 64.5 & 3.6 & 3.7 & $33-36$ & 13,693 \\
\hline Camas Creek & 1,201 & 1,005 & 60.5 & 62 & 3.2 & 3.3 & $22-23$ & 18,223 \\
\hline Herd Creek & 1,047 & 968 & 66.7 & 67.3 & 3.9 & 4.1 & $1-2.5$ & 13,844 \\
\hline Big Creek (upper) & 1,933 & 1,504 & 59.9 & 61.7 & 3.3 & 3.4 & $54-57$ & 35,550 \\
\hline Big Creek (lower) & 943 & 899 & 71.9 & 72.1 & 4.4 & 4.4 & $8-10$ & 21,200 \\
\hline Rush Creek & 59 & 52 & 71.7 & 71.8 & --- & --- & $0-1$ & 1,600 \\
\hline W.F. Chamberlain & 997 & 753 & 61.0 & 63.3 & 3.4 & 3.2 & $1-2$ & 4,500 \\
\hline Chamberlain $\mathrm{Cr}$ & 316 & 243 & 57.9 & 59.4 & 2.7 & 2.8 & $26-26.5$ & 3,594 \\
\hline S.F. Salmon River & 2,608 & 1,490 & 56.9 & 62.3 & 3.1 & 3.2 & $117-118 \& 121-122$ & 38,337 \\
\hline Secesh River & 1,708 & 1,142 & 58.1 & 61.9 & 2.7 & 3.0 & $25-29$ & 41,211 \\
\hline Lake Creek & 1,029 & 664 & 58.0 & 61.5 & 3.1 & 3.2 & $1-2$ & 22,160 \\
\hline Totals or averages & 25,209 & 18,346 & 60.4 & 63.2 & 3.2 & 3.4 & 35 & 304,519 \\
\hline
\end{tabular}


Table 2. Summary of species other than Chinook salmon parr observed during collection operations in Idaho in July and August 2003. Numbers of steelhead in parentheses were PIT tagged for the Idaho Department of Fish and Game.

\begin{tabular}{|c|c|c|c|c|c|c|c|c|c|c|c|}
\hline Stream & Steelhead & $\begin{array}{l}\text { Tagged } \\
\text { steelhead }\end{array}$ & $\begin{array}{l}\text { Unidentified } \\
\text { fry }\end{array}$ & $\begin{array}{c}\text { Brook } \\
\text { trout }\end{array}$ & $\begin{array}{c}\text { Cutthroat } \\
\text { trout }\end{array}$ & Bull trout & Sculpin & Dace & Sucker & Whitefish & Shiner \\
\hline Bear Valley Creek & 16 & $(0)$ & 39 & 24 & 0 & 0 & 1 & 16 & 1 & 598 & 0 \\
\hline Elk Creek & 61 & $(0)$ & 134 & 61 & 1 & 0 & 6 & 80 & 8 & 812 & 0 \\
\hline Marsh Creek & 51 & $(0)$ & 428 & 116 & 1 & 1 & 502 & 0 & 2 & 19 & 0 \\
\hline Sulphur Creek & 6 & $(0)$ & 98 & 0 & 8 & 1 & 11 & 71 & 3 & 38 & 0 \\
\hline Cape Horn Creek & 49 & $(0)$ & 46 & 117 & 0 & 1 & 706 & 0 & 0 & 0 & 0 \\
\hline Valley Creek & 150 & (89) & 337 & 60 & 0 & 2 & 570 & 356 & 232 & 82 & 384 \\
\hline Loon Creek & 98 & (34) & 588 & 0 & 0 & 3 & 0 & 0 & 0 & 14 & 1 \\
\hline Camas Creek & 155 & $(70)$ & 474 & 0 & 0 & 3 & 156 & 0 & 1 & 1 & 0 \\
\hline Herd Creek & 188 & (149) & 0 & 0 & 0 & 3 & 273 & 0 & 0 & 14 & 0 \\
\hline Big Creek (upper) & 142 & $(70)$ & 698 & 167 & 1 & 9 & 2,844 & 3 & 0 & 0 & 0 \\
\hline Big Creek (lower) & 199 & $(126)$ & 408 & 0 & 5 & 2 & 100 & 114 & 25 & 2 & 0 \\
\hline Rush Creek & 90 & $(67)$ & 3 & 0 & 0 & 1 & 22 & 0 & 0 & 21 & 0 \\
\hline W.F. Chamberlain $\mathrm{Cr}$ & 135 & $(56)$ & 281 & 0 & 0 & 5 & 206 & 0 & 0 & 0 & 0 \\
\hline Chamberlain Creek & 116 & $(30)$ & 167 & 0 & 18 & 0 & 94 & 0 & 0 & 1 & 0 \\
\hline S. Fork Salmon River & 294 & $(0)$ & 1,242 & 49 & 0 & 1 & 741 & 22 & 0 & 19 & 0 \\
\hline Secesh River & 105 & $(0)$ & 495 & 14 & 0 & 3 & 453 & 215 & 0 & 5 & 0 \\
\hline Lake Creek & 33 & $(0)$ & 73 & 39 & 0 & 18 & 786 & 0 & 0 & 8 & 0 \\
\hline Totals & 1,888 & (691) & 5,511 & 647 & 34 & 53 & 7,471 & 877 & 272 & 1,634 & 385 \\
\hline
\end{tabular}


Table 3. Mortality percentages for wild Chinook salmon parr collected and PIT-tagged in Idaho in July and August 2003. Loon Creek was the only creek that experienced any tag loss and it was a single tag. None of the other streams sampled had any tag loss in 2003.

\begin{tabular}{lccc}
\hline & \multicolumn{3}{c}{ Mortality } \\
\cline { 2 - 4 } Tagging location & Collection & Tagging \& 24-hour & Overall \\
\hline Bear Valley Creek & 0.0 & 0.0 & 0.4 \\
Elk Creek & 1.3 & 0.1 & 1.5 \\
Marsh Creek & 0.5 & 0.1 & 0.6 \\
Sulphur Creek & 0.1 & 0.5 & 0.5 \\
Cape Horn Creek & 1.2 & 0.0 & 0.9 \\
Valley Creek & 0.6 & 0.2 & 0.8 \\
Loon Creek & 0.4 & 0.1 & 0.5 \\
Camas Creek & 1.2 & 0.2 & 1.5 \\
Herd Creek & 2.7 & 0.0 & 2.7 \\
Big Creek (upper) & 1.3 & 0.0 & 1.3 \\
Big Creek (lower) & 4.7 & 0.0 & 4.7 \\
Rush Creek & 11.9 & 0.0 & 11.9 \\
West Fork Chamberlain Creek & 0.0 & 0.4 & 0.3 \\
Chamberlain Creek & 2.2 & 0.0 & 2.2 \\
South Fork Salmon River & 0.5 & 0.0 & 0.5 \\
Secesh River & 1.1 & 0.3 & 1.3 \\
Lake Creek & 0.1 & 0.0 & 0.1 \\
Totals or averages & 1.0 & 0.1 & 1.1 \\
\hline
\end{tabular}




\section{Detections at In-stream PIT-Tag Monitors}

From 4 to 6 August 2003, 2,498 wild Chinook salmon parr were collected, PIT tagged, and released in natal rearing areas from 3 to $16 \mathrm{~km}$ above the upstream PIT-tag in-stream monitor in lower Valley Creek (Table 1). Between 4 August 2003 and 30 June 2004, the two in-stream detectors (VC1 and VC2) had 251 unique detections of these summer-tagged Chinook salmon juveniles (Figure 2). The upstream monitor (VC1) detected 67 Chinook salmon, and the downstream monitor (VC2) detected 204, of which 184 had not been detected upstream. Average travel time for the 20 fish detected at both monitoring sites was $3 \mathrm{~h}$ and $44 \mathrm{~min}$ (range $19 \mathrm{~min}$ to $17 \mathrm{~h}, 43 \mathrm{~min}$ ). Of the 251 total detections, 148 (59\%) occurred in late-summer/fall (August, September, October); $81(32.3 \%)$ were detected in winter (November, December, January, February); and 22 $(8.8 \%)$ were detected in spring (March, April, May, June) (Figure 2). An estimated 28 to $40 \%$ of all summer-tagged parr survived to migrate out of this stream.

Wild fish passed both monitors predominately during hours of darkness, with over 90\% passing between 1800 and 0600 PST.

\section{Recaptures at Traps and Dams}

A total of 507 wild fish PIT-tagged in summer 2003 were recaptured at traps above Lower Granite Dam from summer-fall 2003 to summer 2004, and 975 were recaptured in the separation-by-code system at the Little Goose Dam juvenile fish facility (Table 4). Weight gain and length increases varied depending on the time between tagging and recapture. 


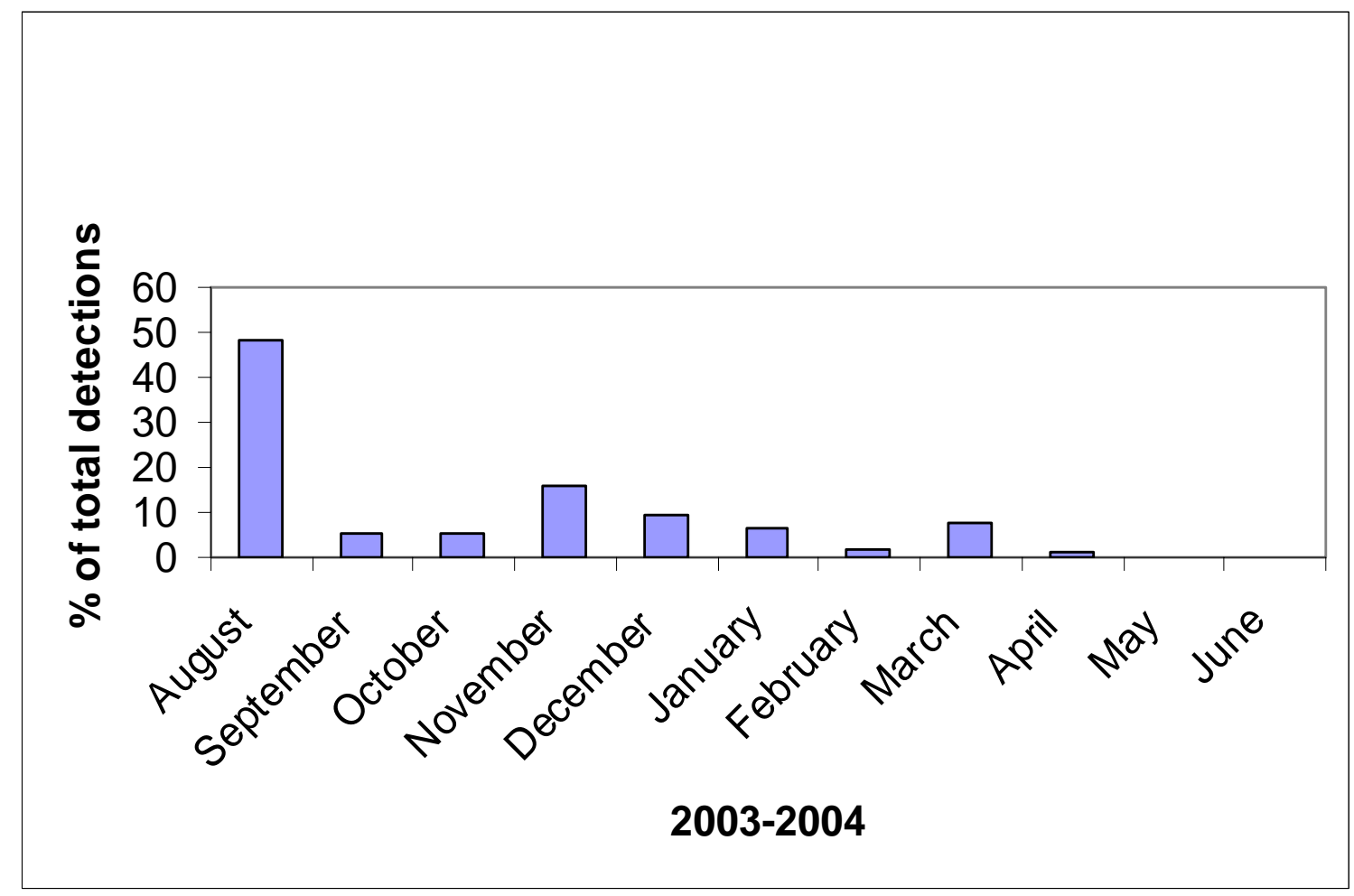

Figure 2. Percent of total detections, by month, of 251 spring/summer Chinook salmon parr, pre-smolts, and smolts detected at the upper and lower in-stream PIT-tag monitoring antennas in lower Valley Creek from August 2003 through June 2004. A total of 2,498 Chinook salmon parr were PIT tagged and released in areas from 3 to $16 \mathrm{~km}$ above these antennas from 4 to 6 August 2003. 
Table 4. Recapture information on PIT-tagged wild spring/summer Chinook salmon from Idaho that were tagged in summer 2003 and recaptured by the separation-by-code system in the juvenile fish bypass system at Little Goose Dam in 2004 and at traps and dams in the summer and fall of 2003 and spring of 2004.

\begin{tabular}{|c|c|c|c|c|c|c|c|c|c|c|c|}
\hline & \multirow{2}{*}{$\begin{array}{c}\text { Number } \\
\text { recaptured }\end{array}$} & \multicolumn{3}{|c|}{$\begin{array}{l}\text { Length gain } \\
\quad(\mathrm{mm})\end{array}$} & \multicolumn{3}{|c|}{$\begin{array}{l}\text { Weight gain } \\
\text { (g) }\end{array}$} & \multicolumn{2}{|c|}{$\begin{array}{l}\text { Condition } \\
\text { factor }\end{array}$} & \multicolumn{2}{|c|}{$\begin{array}{c}\text { Recapture interval } \\
\text { (days) }\end{array}$} \\
\hline & & $\mathrm{n}$ & range & mean & $\mathrm{n}$ & range & mean & release & recapture & range & mean \\
\hline \multicolumn{12}{|c|}{ Streams } \\
\hline Bear Valley Cr. & 68 & 68 & $29-62$ & 43.4 & 47 & $4.3-15.0$ & 8.9 & 1.27 & 0.99 & $267-323$ & 294 \\
\hline Elk Cr. & 79 & 79 & $22-68$ & 44.4 & 48 & 4.6-19.1 & 9.5 & 1.34 & 0.99 & $263-327$ & 295 \\
\hline Sulphur Cr. & 27 & 27 & $21-57$ & 40.5 & 2 & $7.1-8.8$ & 8.0 & 1.37 & 0.99 & $263-301$ & 279 \\
\hline Marsh Cr. & 86 & 86 & $16-61$ & 38.0 & 29 & $1.2-20.3$ & 7.8 & 1.27 & 0.97 & $265-323$ & 284 \\
\hline Cape Horn Cr. & 23 & 23 & $25-50$ & 37.3 & 7 & $5.6-9.2$ & 6.9 & 1.31 & 1.01 & $256-300$ & 278 \\
\hline Valley Cr. & 89 & 89 & $21-69$ & 44.0 & 36 & $2-14.8$ & 9.2 & 1.31 & 1.00 & $261-319$ & 287 \\
\hline Loon Cr. & 96 & 96 & $16-62$ & 41.3 & 54 & $4.3-15.4$ & 8.2 & 1.33 & 0.99 & 253-305 & 277 \\
\hline Camas Cr. & 78 & 78 & $25-65$ & 43.7 & 23 & $2.7-16.3$ & 8.1 & 1.41 & 0.99 & $254-308$ & 279 \\
\hline Herd $\mathrm{Cr}$ & 80 & 80 & $21-63$ & 41.0 & 8 & $5.8-13.2$ & 8.2 & 1.29 & 0.97 & $252-317$ & 270 \\
\hline Big Cr. (upper) & 94 & 94 & $24-64$ & 42.1 & 46 & $3.8-14.4$ & 8.8 & 1.35 & 1.02 & $253-310$ & 280 \\
\hline S. F. Salmon River & 73 & 72 & $14-62$ & 40.3 & 37 & $1.6-15.5$ & 8.1 & 1.22 & 1.01 & $239-307$ & 268 \\
\hline Big Cr. (lower) & 89 & 89 & $20-54$ & 36.8 & 85 & $3.6-15.2$ & 8.1 & 1.17 & 0.96 & $242-276$ & 254 \\
\hline W.F. Chamberlain Cr. & 38 & 38 & $32-57$ & 42.2 & 5 & $6.7-11.6$ & 8.7 & 1.34 & 0.97 & $242-311$ & 262 \\
\hline Secesh River & 33 & 33 & $19-64$ & 41.0 & 21 & $2.7-18.3$ & 8.9 & 1.22 & 0.98 & $234-292$ & 257 \\
\hline Lake Creek. & 22 & 22 & $26-53$ & 38.2 & 14 & $4.2-10.9$ & 7.3 & 1.30 & 1.02 & $235-295$ & 264 \\
\hline Stream totals/averages & 975 & 974 & $14-69$ & 41.3 & 462 & $1.2-20.3$ & 8.5 & 1.28 & 0.99 & $234-327$ & 277 \\
\hline
\end{tabular}


Table 4. Continued.

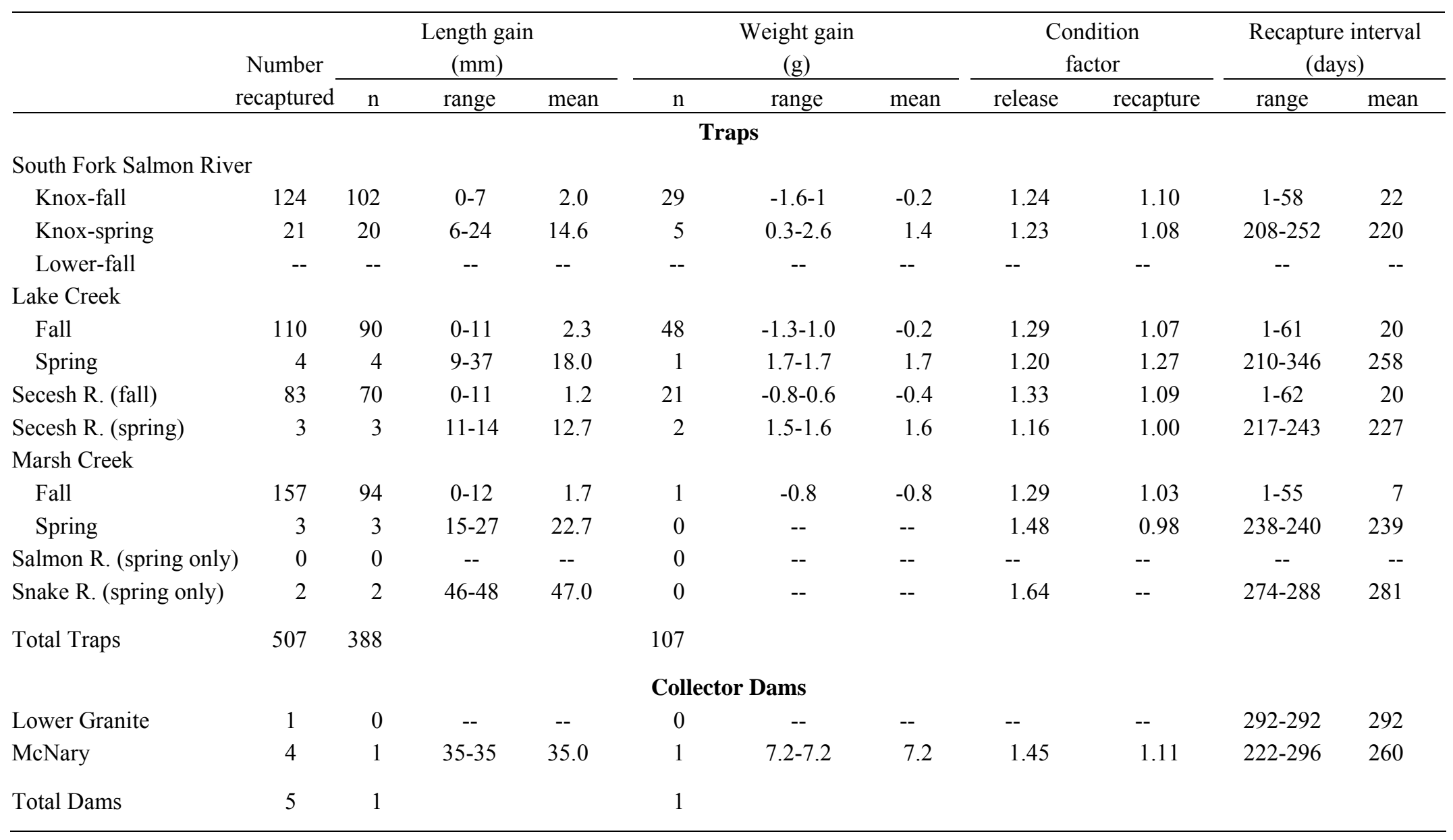




\section{Detections at Dams}

Based on expanded detections (1,472 fish $)^{\dagger}$ at Lower Granite Dam from 1 April to 16 July 2004, estimated survival from parr to smolt for Idaho fish averaged $8.0 \%$ (range 4.1-18.0\%; Table 5; Appendix Tables 4-19). An additional 336 first-time detections (unadjusted) were recorded at Little Goose, Lower Monumental, McNary, John Day, and Bonneville Dams (Appendix Tables 4-18 and 20-22). No first-time detections occurred at the PIT-tag trawl near the mouth of the Columbia River. By comparing all first-time detections at interrogation dams $(1,342)$ to the expanded number of detections at Lower Granite Dam $(1,472)$, we estimated that $9.1 \%$ of the wild fish from Idaho passed through the dams undetected.

For parr tagged in Idaho, average fork length at release was $63.2 \mathrm{~mm}$ (Table 1; Appendix Table 1). However, among fish from this group that were detected the following spring at the dams, average fork length at release was significantly higher ( $65.7 \mathrm{~mm} ; P<0.01)$. The release-length distribution of detected fish was also significantly different from that of released fish in all length categories except $85-89 \mathrm{~mm}$ (but numbers for this length category were too low to evaluate) $(P<0.02$; Figure 3$)$.

We again in 2004 found a significant difference in fork lengths at time of release for fish that migrated through Lower Granite Dam in April and May compared to fish that migrated after May $(P<0.01)$. Fish migrating through the dam in April and May were on average $3.7 \mathrm{~mm}$ larger when released than fish migrating after May. These data suggest that fish size influences migration timing or overwinter location.

In 2004, for the first time we were able to look at estimated survival rates to Lower Granite Dam of juveniles previously passively monitored leaving a stream. We estimated a $13.2 \%$ overall survival rate to the dam for Chinook salmon juveniles previously detected at the Valley Creek in-stream PIT-tag monitors. The overall parr-to-smolt estimated survival rate for fish from this stream was 5.5\% (Table 5). The estimated survival rates for the various groups of fish leaving this stream in 2003-2004 were $6.6 \%$ for fish leaving the stream in late-summer/fall, $16.9 \%$ for fish leaving the stream in winter, and $43.9 \%$ for fish leaving the stream in spring.

$\dagger$ Due to rounding of numbers, the expanded detection numbers at Lower Granite Dam in Table 5 may vary slightly from expanded detection numbers in Appendix Tables 4-19. 
Table 5. Summary of observed and expanded detections of PIT-tagged wild spring/summer Chinook salmon smolts from Idaho at Lower Granite Dam in 2004. Expanded numbers used for parr-to-smolt survival estimates are shown with standard errors in parenthesis. See Table 1 for numbers released.

\begin{tabular}{|c|c|c|c|c|c|}
\hline \multirow[b]{3}{*}{ Stream } & \multicolumn{5}{|c|}{ Lower Granite Dam Detections } \\
\hline & \multicolumn{2}{|c|}{ Observed } & \multicolumn{3}{|c|}{ Expanded } \\
\hline & $\mathrm{N}$ & $\%$ & $\mathrm{~N}$ & $\%$ & SE $(\%)$ \\
\hline Bear Valley Creek & 63 & 4.2 & 86 & 5.8 & $(1.0)$ \\
\hline Elk Creek & 83 & 5.5 & 117 & 7.7 & $(1.0)$ \\
\hline Marsh Creek & 83 & 5.4 & 129 & 8.4 & $(1.0)$ \\
\hline Cape Horn Creek & 26 & 3.9 & 39 & 5.8 & $(1.0)$ \\
\hline Sulphur Creek & 26 & 2.5 & 43 & 4.1 & $(1.0)$ \\
\hline Valley Creek & 108 & 4.3 & 138 & 5.5 & $(1.0)$ \\
\hline Loon Creek & 91 & 10.6 & 118 & 13.7 & $(2.0)$ \\
\hline Camas Creek & 73 & 7.3 & 101 & 10.0 & $(1.0)$ \\
\hline Herd Creek & 81 & 8.4 & 111 & 11.5 & $(2.0)$ \\
\hline Big Creek (upper) & 100 & 6.6 & 140 & 9.3 & $(1.0)$ \\
\hline Big (lower)/Rush Creeks & 95 & 10.0 & 171 & 18.0 & $(3.0)$ \\
\hline W. F. Chamberlain Creek* & 48 & 4.8 & 82 & 8.2 & $(2.0)$ \\
\hline S. Fork Salmon River & 73 & 4.9 & 103 & 6.9 & $(1.0)$ \\
\hline Secesh River & 30 & 2.6 & 49 & 4.3 & $(1.0)$ \\
\hline Lake Creek & 26 & 3.9 & 45 & 6.8 & $(2.0)$ \\
\hline Totals or averages & 1,006 & 5.5 & 1,472 & 8.0 & $(0.4)$ \\
\hline
\end{tabular}

* Includes fish from Chamberlain Creek. 


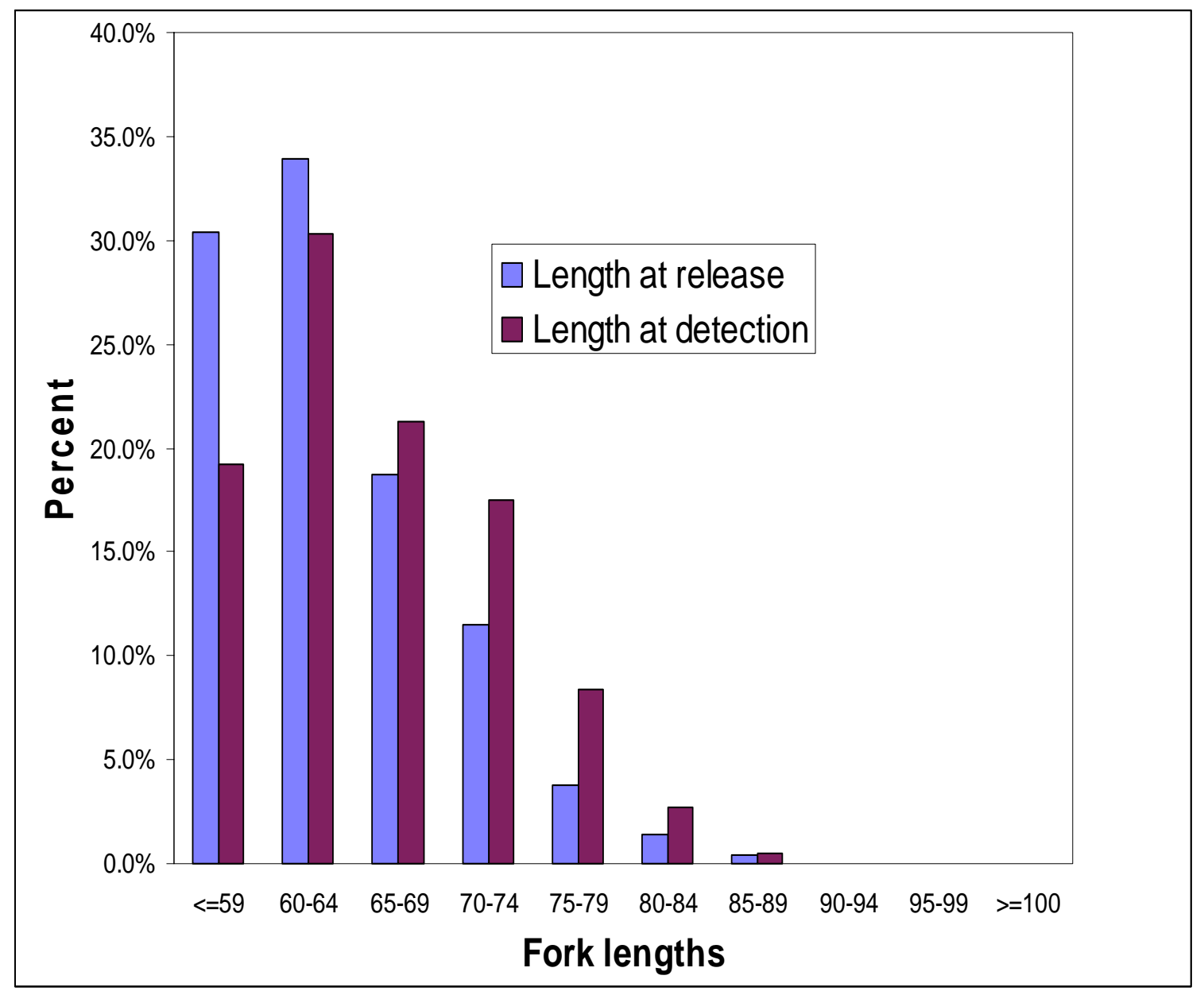

Figure 3. Percent by fork length increments, of PIT-tagged wild spring/summer Chinook salmon parr released in Idaho streams in $2003(\mathrm{n}=18,307)$ and percent of fish detected for these length increments at dams in spring and summer 2004 $(\mathrm{n}=1,341)$. 


\section{Migration Timing}

\section{Lower Granite Dam}

Passage timing at Lower Granite Dam varied for fish from the 20 Idaho and Oregon streams (Figure 4). In comparisons among all 20 Idaho and Oregon streams (Tables 6a-6b, Figure 4), fish from the Secesh River had a significantly earlier timing for 10th percentile passage than fish from all the other streams $(P<0.05)$. The 10th percentile passage date of fish from Valley Creek was significantly later than that of fish from all other streams except Loon, Camas, Elk, Big (upper), and Catherine Creeks and the Imnaha River $(P<0.05)$. The SEs on these passage estimates ranged from 0.1 to $5.2 \mathrm{~d}$ (median $1.8 \mathrm{~d}$ ). Overall, the 10th percentile passage dates for fish from all 20 streams ranged from 4 April to 25 April (Tables 6a-6b).

In comparisons of the 50th percentile passage date at the dam, fish from Big (lower)/Rush Creeks were significantly earlier than fish from all other streams except Chamberlain/W. F. Chamberlain Creeks, Lake, Sulphur, Marsh, and Herd Creeks, and the Minam and Secesh Rivers $(P<0.05)$. Fish from Catherine Creek were significantly later than fish from all other streams except Big (upper), Valley, Elk, Camas, and Bear Valley Creeks and the South Fork Salmon River $(P<0.05)$. The SEs on these passage estimates ranged from 0.5 to $5.8 \mathrm{~d}$ (median $2.2 \mathrm{~d}$ ). The overall 50 th percentile passage dates for fish from all 20 streams ranged from 23 April to 15 May (Tables 6a-6b).

In terms of the 90th percentile passage date at the dam, fish from $\operatorname{Big}($ lower)/Rush Creeks were significantly earlier than fish from all other streams except Chamberlain/W. F. Chamberlain Creeks, Herd, Marsh, Sulphur, and Loon Creeks $(P<0.05)$. Fish from Catherine Creek were significantly later than fish from all other streams except Big (upper) and Elk Creeks, and Lostine and South Fork Salmon Rivers. The SEs on these passage estimates ranged from 0.9 to $6.6 \mathrm{~d}$ (median $3.1 \mathrm{~d}$ ). The overall 90th percentile passage dates for fish from all streams ranged from 4 May to 11 June (Tables 6a-6b).

For the number of days encompassing the middle 80th percentile passage (10th to 90th percentile), $\operatorname{Big}($ lower)/Rush Creeks fish had a significantly more condensed distribution, (19 d) than fish from all other streams except Chamberlain/W. F. Chamberlain Creeks, Loon, Herd, Marsh, Sulphur, and Valley Creeks, and the Imnaha River (22-33 d; $P<0.05$; Tables $6 \mathrm{a}-6 \mathrm{~b}$ ). Timing of the middle 80 th percentile passage for Secesh River fish was significantly more protracted (54 d) than for fish from all other streams except Cape Horn, Bear Valley, Lake, Elk, Big (upper), and Catherine Creeks, and Minam, South Fork Salmon, and Lostine Rivers (38-51 d; $P<0.05$; Tables 6a-6b). The SEs for these passage estimates range from 0.9 to $8.0 \mathrm{~d}$ (median $3.8 \mathrm{~d}$ ). 


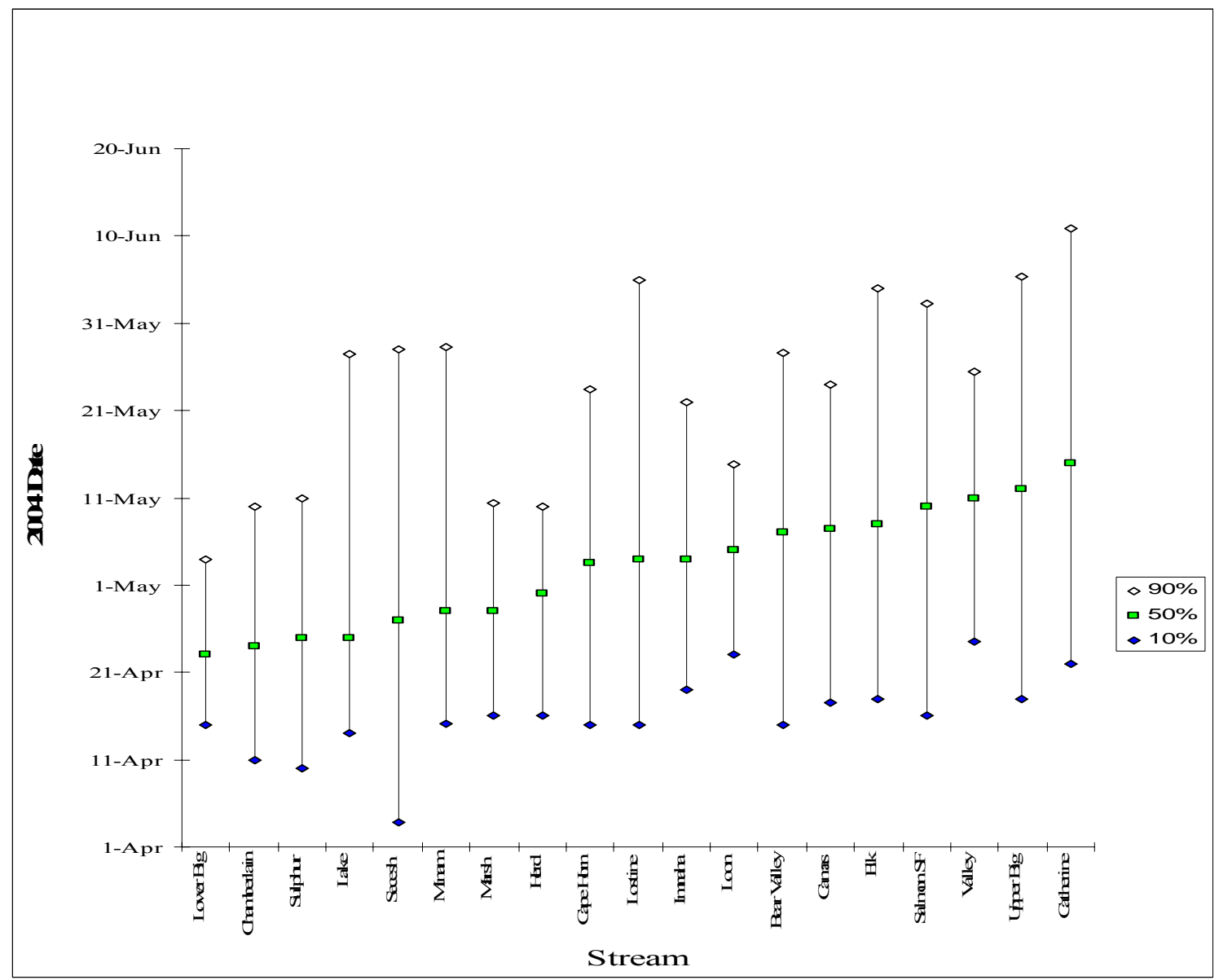

Figure 4. Estimated passage distributions at Lower Granite Dam for wild spring/summer Chinook salmon smolts from streams of Idaho and Oregon in 2004.

Chamberlain and West Fork Chamberlain Creeks are combined and Big Creek is divided into lower and upper portions for these analyses. See Appendix Tables 4-18 for daily estimated passage numbers from Idaho streams at the dam. 
Table 6a. Accumulated and 2004 passage dates at Lower Granite Dam for PIT-tagged wild spring/summer Chinook salmon smolts from streams in Idaho.

\begin{tabular}{|c|c|c|c|c|}
\hline \multirow[b]{2}{*}{ Year } & \multicolumn{4}{|c|}{ Percentile passage dates at Lower Granite Dam } \\
\hline & 10th & 50 th & 90th & Range \\
\hline \multicolumn{5}{|c|}{ Bear Valley Creek } \\
\hline $1990^{\mathrm{a}}$ & 19 April & 05 May & 31 May & 11 April-18 July \\
\hline 1991 & 03 May & 20 May & 12 June & 18 April-23 June \\
\hline 1992 & 15 April & 02 May & 24 May & 07 April-28 June \\
\hline 1993 & 29 April & 16 May & 22 June & 22 April-27 July \\
\hline 1994 & 22 April & 06 May & 29 May & 16 April-15 July \\
\hline 1995 & 28 April & 18 May & 12 June & 13 April-20 July \\
\hline $1996^{\mathrm{a}}$ & --- & --- & --- & --- \\
\hline $1997^{\mathrm{a}}$ & --- & --- & --- & --- \\
\hline 1998 & 25 April & 06 May & 23 May & 31 March-25 June \\
\hline 1999 & 23 April & 03 May & 07 June & 20 April-21 June \\
\hline 2000 & 18 April & 07 May & 02 June & 14 April-02 July \\
\hline 2001 & 08 May & 16 May & 28 May & 26 April-17 June \\
\hline 2002 & 16 April & 04 May & 31 May & 12 April-26 June \\
\hline 2003 & 14 April & 05 May & 28 May & 12 April-14 June \\
\hline 2004 & 15 April & 07 May & 28 May & 13 April-05 July \\
\hline \multicolumn{5}{|c|}{ Elk Creek } \\
\hline $1990^{\mathrm{a}}$ & --- & --- & --- & --- \\
\hline 1991 & 03 May & 20 May & 16 June & 25 April-24 June \\
\hline 1992 & 11 April & 30 April & 28 May & 05 April-17 July \\
\hline 1993 & 02 May & 16 May & 11 June & 21 April-26 June \\
\hline 1994 & 23 April & 04 May & 21 May & 18 April-09 July \\
\hline 1995 & 18 April & 11 May & 05 June & 10 April-09 July \\
\hline $1996^{\mathrm{a}}$ & --- & --- & --- & - \\
\hline $1997^{\mathrm{a}}$ & --- & --- & --- & --- \\
\hline 1998 & 07 April & 02 May & 15 May & 04 April-21 June \\
\hline 1999 & 21 April & 03 May & 27 May & 01 April-08 July \\
\hline 2000 & 15 April & 28 April & 19 May & 13 April-28 May \\
\hline 2001 & 30 April & 11 May & 27 May & 30 April-27 May \\
\hline 2002 & 16 April & 29 April & 02 June & 13 April-05 July \\
\hline 2003 & 20 April & 06 May & 29 May & 31 March-30 May \\
\hline 2004 & 18 April & 08 May & 04 July & 14 April-July 12 \\
\hline \multicolumn{5}{|c|}{ Sulphur Creek } \\
\hline 1990 & 18 April & 30 April & 31 May & 11 April-27 June \\
\hline $1991^{\mathrm{a}}$ & --- & --- & --- & --- \\
\hline 1992 & 16 April & 03 May & 23 May & 10 April-01 June \\
\hline 1993 & 28 April & 16 May & 12 June & 24 April-28 June \\
\hline $1994^{\mathrm{a}}$ & --- & --- & --- & -- \\
\hline 1995 & 02 May & 23 May & 09 June & 11 April-09 July \\
\hline $1996^{\mathrm{a}}$ & --- & --- & --- & --- \\
\hline $1997^{\mathrm{a}}$ & --- & --- & --- & --- \\
\hline 1998 & --- & --- & --- & --- \\
\hline 1999 & 24 April & 19 May & 27 May & 22 April-29 may \\
\hline 2000 & 15 April & 07 May & 24 May & 12 April-30 May \\
\hline $2001^{\mathrm{a}}$ & --- & --- & --- & $1+--$ \\
\hline $2002^{\mathrm{a}}$ & --- & --- & --- & --- \\
\hline 2003 & 02 May & 25 May & 08 June & 22 April-24 June \\
\hline 2004 & 10 April & 25 April & 11 May & 02 April-24 May \\
\hline
\end{tabular}


Table 6a. Continued.

\begin{tabular}{|c|c|c|c|c|}
\hline \multirow[b]{2}{*}{ Year } & \multicolumn{4}{|c|}{ Percentile passage dates at Lower Granite Dam } \\
\hline & 10th & 50th & 90th & Range \\
\hline \multicolumn{5}{|c|}{ Cape Horn Creek } \\
\hline $1990^{\mathrm{a}}$ & --- & --- & --- & --- \\
\hline 1991 & 24 April & 16 May & 28 May & 19 April-06 June \\
\hline 1992 & 12 April & 28 April & 30 May & 10 April-01 June \\
\hline 1993 & 08 May & 19 May & 26 June & 05 May-01 July \\
\hline $1994^{\mathrm{a}}$ & --- & --- & --- & --- \\
\hline 1995 & 29 April & 14 May & 19 June & 14 April-28 July \\
\hline $1996^{\mathrm{a}}$ & --- & --- & --- & --- \\
\hline $1997^{\mathrm{a}}$ & --- & --- & --- & --- \\
\hline $1998^{\mathrm{a}}$ & --- & --- & --- & --- \\
\hline 1999 & 29 April & 22 May & 29 May & 25 April-12 June \\
\hline 2000 & 01 May & 24 May & 01 June & 20 April-09 July \\
\hline $2001^{\mathrm{a}}$ & --- & --- & --- & I --- \\
\hline $2002^{a}$ & --- & --- & --- & --- \\
\hline 2003 & 21 April & 17 May & 01 June & 15 April-18 June \\
\hline 2004 & 15 April & 04 May & 24 May & 14 April-28 May \\
\hline \multicolumn{5}{|c|}{ Camas Creek } \\
\hline 1993 & 03 May & 16 May & 27 May & 24 April-24 June \\
\hline 1994 & 30 April & 15 May & 26 May & 24 April-11 July \\
\hline 1995 & 27 April & 12 May & 05 June & 17 April-11 June \\
\hline $1996^{\mathrm{a}}$ & --- & --- & --- & -- \\
\hline $1997^{\mathrm{a}}$ & --- & --- & --- & --- \\
\hline $1998^{\mathrm{a}}$ & --- & --- & --- & --- \\
\hline $1999^{\mathrm{a}}$ & --- & --- & --- & --- \\
\hline 2000 & 26 April & 25 May & 02 June & 13 April-24 June \\
\hline $2001^{\mathrm{a}}$ & --- & --- & --- & - \\
\hline $2002^{\mathrm{a}}$ & --- & --- & --- & --- \\
\hline 2003 & 02 May & 24 May & 30 May & 26 April-06 June \\
\hline 2004 & 18 April & 08 May & 24 May & 16 April-04 June \\
\hline \multicolumn{5}{|c|}{ Marsh Creek } \\
\hline 1990 & 17 April & 29 April & 31 May & 09 April-01 July \\
\hline 1991 & 26 April & 20 May & 09 June & 17 April-18 June \\
\hline 1992 & 17 April & 07 May & 02 June & 10 April-13 July \\
\hline 1993 & 29 April & 15 May & 27 May & 24 April-10 August \\
\hline 1994 & 23 April & 04 May & 18 May & 16 April-08 August \\
\hline 1995 & 17 April & 09 May & 24 May & 11 April-08 July \\
\hline $1996^{\mathrm{a}}$ & --- & --- & --- & --- \\
\hline $1997^{\mathrm{a}}$ & --- & --- & --- & --- \\
\hline $1998^{\mathrm{a}}$ & --- & --- & --- & --- \\
\hline 1999 & 21 April & 01 May & 25 May & 11 April-13 June \\
\hline 2000 & 21 April & 28 April & 27 May & 14 April-16 June \\
\hline $2001^{\mathrm{a}}$ & --- & --- & --- & --- \\
\hline 2002 & 18 April & 04 May & 23 May & 14 April-26 May \\
\hline 2003 & 14 April & 05 May & 29 May & 03 April-09 June \\
\hline 2004 & 16 April & 28 April & 10 May & 03 April-30 May \\
\hline
\end{tabular}


Table 6a. Continued.

\begin{tabular}{|c|c|c|c|c|}
\hline \multirow[b]{2}{*}{ Year } & \multicolumn{4}{|c|}{ Percentile passage dates at Lower Granite Dam } \\
\hline & 10th & 50 th & 90th & Range \\
\hline \multicolumn{5}{|c|}{ Valley Creek } \\
\hline 1989 & 24 April & 14 May & 12 June & 09 April-17 June \\
\hline 1990 & 16 April & 08 May & 05 June & 12 April-29 June \\
\hline 1991 & 11 May & 20 May & 20 June & 21 April-13 July \\
\hline 1992 & 15 April & 30 April & 27 May & 13 April-04 June \\
\hline 1993 & 30 April & 16 May & 02 June & 24 April-06 June \\
\hline 1994 & 24 April & 04 May & 03 June & 22 April-09 June \\
\hline 1995 & 04 May & 02 June & 08 July & 22 April-18 July \\
\hline $1996^{\mathrm{a}}$ & --- & --- & -- & --- \\
\hline $1997^{\mathrm{a}}$ & --- & --- & --- & --- \\
\hline $1998^{\mathrm{a}}$ & --- & --- & --- & --- \\
\hline 1999 & 24 April & 13 May & 12 June & 19 April-01 July \\
\hline 2000 & 20 April & 12 May & 29 May & 13 April-14 July \\
\hline 2001 & 10 May & 19 May & 01 June & 28 April-03 July \\
\hline 2002 & 24 April & 20 May & 03 June & 19 April-19 June \\
\hline 2003 & 14 April & 17 May & 28 May & 01 April-31 May \\
\hline 2004 & 25 April & 11 May & 26 May & 04 April-16 June \\
\hline \multicolumn{5}{|c|}{ Loon Creek } \\
\hline 1993 & 05 May & 12 May & 17 May & 03 May-5 June \\
\hline 1994 & 29 April & 10 May & 24 May & 22 April-07 June \\
\hline 1995 & 23 April & 11 May & 28 May & 13 April-07 June \\
\hline $1996^{\mathrm{a}}$ & --- & --- & --- & --- \\
\hline $1997^{\mathrm{a}}$ & --- & --- & --- & --- \\
\hline $1998^{\mathrm{a}}$ & --- & --- & --- & --- \\
\hline 1999 & 30 April & 18 May & 27 May & 22 April-16 June \\
\hline 2000 & 22 April & 08 May & 24 May & 14 April-01 June \\
\hline $2001^{\mathrm{a}}$ & --- & --- & --- & --- \\
\hline $2002^{a}$ & --- & --- & --- & --- \\
\hline 2003 & 30 April & 17 May & 28 May & 21 April-30 May \\
\hline 2004 & 23 April & 05 May & 15 May & 15 April-26 May \\
\hline \multicolumn{5}{|c|}{ East Fork Salmon River } \\
\hline 1989 & 22 April & 03 May & 18 May & 07 April-08 June \\
\hline $1990^{\mathrm{a}}$ & --- & --- & --- & --- \\
\hline 1991 & 22 April & 09 May & 26 May & 16 April-20 June \\
\hline 1992 & 13 April & 21 April & 16 May & 10 April-03 June \\
\hline 1993 & 25 April & 06 May & 18 May & 22 April-01 June \\
\hline 1994 & 22 April & 28 April & 17 May & 20 April-25 May \\
\hline 1995 & 14 April & 28 April & 10 May & 11 April-27 May \\
\hline $1996^{\mathrm{a}}$ & --- & --- & --- & --- \\
\hline $1997^{\mathrm{a}}$ & --- & --- & --- & --- \\
\hline $1998^{\mathrm{a}}$ & --- & --- & --- & --- \\
\hline $1999^{\mathrm{a}}$ & --- & --- & --- & --- \\
\hline 2000 & 21 April & 07 May & 25 May & 15 April-27 May \\
\hline $2001^{\mathrm{a}}$ & --- & --- & --- & --- \\
\hline $2002^{a}$ & --- & --- & --- & --- \\
\hline $2003^{a}$ & --- & --- & --- & --- \\
\hline $2004^{\mathrm{a}}$ & --- & --- & --- & --- \\
\hline
\end{tabular}


Table 6a. Continued.

\begin{tabular}{|c|c|c|c|c|}
\hline \multirow[b]{2}{*}{ Year } & \multicolumn{4}{|c|}{ Percentile passage dates at Lower Granite Dam } \\
\hline & 10th & 50th & 90th & Range \\
\hline \multicolumn{5}{|c|}{ Herd Creek } \\
\hline 1992 & 14 April & 20 April & 10 May & 13 April-18 May \\
\hline 1993 & 26 April & 30 April & 18 May & 26 April-31 May \\
\hline $1994^{\mathrm{b}}$ & --- & --- & --- & --- \\
\hline 1995 & 18 April & 03 May & 14 May & 11 April-28 May \\
\hline $1996^{\mathrm{a}}$ & --- & --- & --- & --- \\
\hline $1997^{\mathrm{a}}$ & --- & --- & --- & --- \\
\hline $1998^{\mathrm{a}}$ & --- & --- & --- & --- \\
\hline 1999 & 20 April & 29 April & 10 May & 30 March-20 May \\
\hline 2000 & 16 April & 25 April & 18 May & 14 April-19 May \\
\hline 2001 & 30 April & 04 May & 14 May & 28 April-07 June \\
\hline $2002^{b}$ & --- & --- & --- & --- \\
\hline 2003 & 16 April & 03 May & 26 May & 06 April-29 May \\
\hline 2004 & 16 April & 30 April & 10 May & 12 April-21 June \\
\hline \multicolumn{5}{|c|}{ South Fork Salmon River } \\
\hline 1989 & 25 April & 13 May & 14 June & 16 April-20 June \\
\hline $1990^{\mathrm{a}}$ & --- & --- & --- & --- \\
\hline 1991 & 20 April & 16 May & 10 June & 17 April-13 July \\
\hline 1992 & 14 April & 29 April & 27 May & 07 April-27 July \\
\hline 1993 & 29 April & 16 May & 02 June & 26 April-28 June \\
\hline 1994 & 27 April & 15 May & 28 June & 22 April-09 July \\
\hline 1995 & 20 April & 10 May & 10 June & 13 April-13 July \\
\hline 1996 & 19 April & 15 May & 09 June & 19 April-03 July \\
\hline 1997 & 13 April & 28 April & 12 June & 07 April-15 June \\
\hline 1998 & 25 April & 12 May & 15 June & 02 April-07 August \\
\hline 1999 & $31 \mathrm{March}$ & 04 May & 01 June & 27 March-11 June \\
\hline 2000 & 20 April & 18 May & 31 May & 12 April-20 July \\
\hline 2001 & 29 April & 14 May & 01 June & 26 April-07 July \\
\hline 2002 & 15 April & 03 May & 24 May & 11 April-09 June \\
\hline 2003 & 19 April & 16 May & 03 June & 19 April-12 June \\
\hline 2004 & $16 \mathrm{Apr}$ & 10 May & 02 June & 08 April-19 June \\
\hline \multicolumn{5}{|c|}{ Big Creek (upper) } \\
\hline 1990 & 27 April & 30 May & 22 June & 17 April-18 July \\
\hline 1991 & 18 May & 10 June & 26 June & 26 April-01 July \\
\hline 1992 & 22 April & 08 May & 03 June & 15 April-26 June \\
\hline 1993 & 08 May & 18 May & 26 May & 26 April-15 June \\
\hline 1994 & 03 May & 19 May & 19 July & 25 April-30 August \\
\hline 1995 & 05 May & 23 May & 09 June & 02 May-26 June \\
\hline $1996^{\mathrm{a}}$ & --- & --- & --- & --- \\
\hline $1997^{\mathrm{a}}$ & --- & --- & --- & --- \\
\hline $1998^{\mathrm{a}}$ & --- & --- & --- & --- \\
\hline 1999 & 28 April & 14 May & 03 June & 25 April-19 June \\
\hline 2000 & 30 April & 27 May & 14 June & 15 April-29 June \\
\hline $2001^{\mathrm{a}}$ & --- & --- & --- & --- \\
\hline $2002^{\mathrm{a}}$ & --- & --- & --- & --- \\
\hline 2003 & 06 May & 25 May & 01 June & 01 May-21 June \\
\hline 2004 & 18 April & 12 May & 05 June & 15 April-17 June \\
\hline
\end{tabular}


Table 6a. Continued.

\begin{tabular}{|c|c|c|c|c|}
\hline \multirow[b]{2}{*}{ Year } & \multicolumn{4}{|c|}{ Percentile passage dates at Lower Granite Dam } \\
\hline & 10th & 50th & 90th & Range \\
\hline \multicolumn{5}{|c|}{ Big (lower)/Rush Creeks } \\
\hline 1993 & 24 April & 29 April & 13 May & 21 April-16 May \\
\hline 1994 & 23 April & 29 April & 11 May & 21 April-15 June \\
\hline 1995 & 19 April & 01 May & 14 May & 11 April-05 June \\
\hline $1996^{\mathrm{a}}$ & --- & --- & --- & --- \\
\hline $1997^{\mathrm{a}}$ & --- & --- & --- & --- \\
\hline $1998^{\mathrm{a}}$ & --- & --- & --- & --- \\
\hline 1999 & 19 April & 28 April & 23 May & 04 April-30 May \\
\hline 2000 & 19 April & 30 April & 13 May & 16 April-26 May \\
\hline $2001^{\mathrm{a}}$ & --- & --- & --- & --- \\
\hline 2002 & 15 April & 25 April & 07 May & 12 April-22 May \\
\hline 2003 & 14 April & 26 April & 18 May & 12 April-25 May \\
\hline 2004 & 15 April & 23 April & 04 May & 06 April-15 May \\
\hline \multicolumn{5}{|c|}{ West Fork Chamberlain Creek } \\
\hline $1992^{\mathrm{c}}$ & 15 April & 26 April & 03 June & 12 April-24 June \\
\hline 1993 & 28 April & 15 May & 23 June & 23 April-22 July \\
\hline $1994^{\mathrm{c}}$ & 24 April & 01 May & 05 July & 24 April-04 September \\
\hline $1995^{\mathrm{c}}$ & 16 April & 09 May & 20 June & 12 April-22 September \\
\hline $1996^{\mathrm{a}}$ & --- & --- & --- & 1 $\quad--$ \\
\hline $1997^{\mathrm{a}}$ & --- & --- & --- & --- \\
\hline $1998^{\mathrm{a}}$ & --- & --- & --- & --- \\
\hline $1999^{\mathrm{a}}$ & --- & --- & --- & --- \\
\hline $2000^{\mathrm{a}}$ & --- & --- & --- & --- \\
\hline $2001^{\mathrm{a}}$ & --- & --- & --- & --- \\
\hline 2002 & 26 April & 04 May & 20 May & 18 April-29 May \\
\hline $2003^{c}$ & 23 April & 20 May & 26 May & 21 April-26 May \\
\hline $2004^{c}$ & 11 April & 24 April & 10 May & 07 April-23 June \\
\hline \multicolumn{5}{|c|}{ Secesh River } \\
\hline 1989 & 20 April & 27 April & 09 June & 09 April-19 July \\
\hline 1990 & 14 April & 22 April & 07 June & 10 April-13 July \\
\hline 1991 & 20 April & 27 April & 14 June & 13 April-20 July \\
\hline 1992 & 13 April & 29 April & 04 June & 05 April-03 July \\
\hline 1993 & 26 April & 16 May & 16 June & 22 April-15 July \\
\hline 1994 & 22 April & 26 April & 11 July & 21 April-07 August \\
\hline 1995 & 14 April & 01 May & 24 May & 10 April-10 July \\
\hline 1996 & 14 April & 25 April & 29 May & 12 April-15 July \\
\hline 1997 & 10 April & 18 April & 04 May & 04 April-11 July \\
\hline 1998 & 08 April & 24 April & 28 May & 03 April-06 July \\
\hline 1999 & 03 April & 23 April & 25 May & 29 March-21 June \\
\hline 2000 & 13 April & 23 April & 04 June & 12 April-11 July \\
\hline 2001 & 16 April & 28 April & 13 May & 06 April-13 June \\
\hline 2002 & 13 April & 21 April & 17 May & 11 April-01 July \\
\hline 2003 & 18 April & 30 April & 01 June & 03 April-04 July \\
\hline 2004 & 04 April & 27 April & 28 May & 01 April-13 June \\
\hline
\end{tabular}


Table 6a. Continued.

\begin{tabular}{|c|c|c|c|c|}
\hline \multirow[b]{2}{*}{ Year } & \multicolumn{4}{|c|}{ Percentile passage dates at Lower Granite Dam } \\
\hline & 10th & 50th & 90th & Range \\
\hline \multicolumn{5}{|c|}{ Lake Creek } \\
\hline 1989 & 23 April & 02 May & 16 June & 12 April-01 July \\
\hline $1990^{\mathrm{a}}$ & --- & --- & --- & --- \\
\hline $1991^{\mathrm{a}}$ & --- & --- & --- & --- \\
\hline $1992^{\mathrm{a}}$ & --- & --- & --- & --- \\
\hline 1993 & 23 April & 09 May & 22 June & 22 April-25 June \\
\hline 1994 & 21 April & 28 April & 19 May & 20 April-24 June \\
\hline 1995 & 17 April & 10 May & 10 June & 14 April-20 July \\
\hline 1996 & 15 April & 21 April & 19 May & 15 April-02 June \\
\hline 1997 & 11 April & 25 April & 02 July & 07 April-22 September \\
\hline 1998 & 04 April & 25 April & 26 May & 02 April-16 July \\
\hline 1999 & 20 April & 26 April & 27 May & 08 April-20 June \\
\hline 2000 & 13 April & 04 May & 04 June & 13 April-18 July \\
\hline $2001^{\mathrm{a}}$ & --- & --- & --- & --- \\
\hline 2002 & 16 April & 29 April & 03 June & 13 April-03 June \\
\hline 2003 & 06 April & 06 May & 04 June & 06 April-20 June \\
\hline 2004 & 14 April & 25 April & 28 May & 09 April-16 June \\
\hline
\end{tabular}

${ }^{\mathrm{a}}$ No parr were tagged the summer prior to this migration year.

${ }^{\mathrm{b}}$ Insufficient numbers detected to estimate timing.

${ }^{\mathrm{c}}$ Includes fish from Chamberlain Creek. 
Table 6b. Accumulated and 2004 passage dates at Lower Granite Dam for PIT-tagged wild spring/summer Chinook salmon smolts from streams in Oregon.

\begin{tabular}{|c|c|c|c|c|}
\hline \multirow[b]{2}{*}{ Year } & \multicolumn{4}{|c|}{ Percentile passage dates at Lower Granite Dam } \\
\hline & 10 th & 50 th & 90th & Range \\
\hline \multicolumn{5}{|c|}{ Catherine Creek } \\
\hline 1991 & 01 May & 14 May & 08 June & 17 April-23 June \\
\hline 1992 & 16 April & 01 May & 21 May & 09 April-29 June \\
\hline 1993 & 06 May & 18 May & 05 June & 29 April-26 June \\
\hline 1994 & 25 April & 11 May & 20 May & 13 April-26 July \\
\hline 1995 & 01 May & 19 May & 09 June & 26 April-02 July \\
\hline $1996^{\mathrm{a}}$ & 19 April & 13 May & 29 May & 14 April-14 June \\
\hline 1997 & 08 May & 14 May & 01 June & 24 April-10 June \\
\hline 1998 & 28 April & 21 May & 28 May & 24 April-04 June \\
\hline 1999 & 26 April & 25 May & 15 June & 26 April-26 June \\
\hline 2000 & 30 April & 08 May & 23 May & 12 April-06 June \\
\hline 2001 & 29 April & 17 May & 17 June & 28 April-03 July \\
\hline 2002 & 24 April & 10 May & 18 June & 15 April-01 July \\
\hline 2003 & 26 April & 10 May & 09 June & 14 April-09 June \\
\hline 2004 & 22 April & 15 May & 11 June & 15 April-25 June \\
\hline \multicolumn{5}{|c|}{ Grande Ronde River (upper) } \\
\hline 1989 & 12 May & 06 June & 19 June & 27 April-22 July \\
\hline $1990^{\mathrm{b}}$ & --- & --- & --- & --- \\
\hline $1991^{\mathrm{b}}$ & --- & --- & --- & --- \\
\hline $1992^{b}$ & --- & --- & --- & --- \\
\hline 1993 & 05 May & 16 May & 25 May & 23 April-20 June \\
\hline 1994 & 28 April & 23 May & 07 July & 23 April-29 August \\
\hline 1995 & 27 April & 29 May & 12 June & 12 April-01 July \\
\hline $1996 \mathrm{c}$ & 26 April & 17 May & 29 May & 19 April-06 June \\
\hline $1997^{\mathrm{b}}$ & --- & --- & --- & --- \\
\hline $1998^{b}$ & --- & --- & --- & --- \\
\hline $1999^{b}$ & --- & --- & --- & --- \\
\hline $2000^{\mathrm{b}}$ & --- & --- & --- & --- \\
\hline $2001^{b}$ & --- & --- & --- & --- \\
\hline $2002^{b}$ & --- & --- & --- & --- \\
\hline $2003^{b}$ & --- & --- & --- & --- \\
\hline 2004 & --- & --- & --- & --- \\
\hline \multicolumn{5}{|c|}{ Imnaha River (lower) } \\
\hline 1989 & 11 April & 30 April & 11 May & 04 April-05 June \\
\hline 1990 & 10 April & 18 April & 09 May & 05 April-27 May \\
\hline 1991 & 20 April & 01 May & 13 May & 14 April-15 May \\
\hline 1992 & 10 April & 21 April & 03 May & 06 April-21 May \\
\hline $1993^{b}$ & --- & --- & --- & --- \\
\hline $1994^{\mathrm{b}}$ & --- & --- & --- & --- \\
\hline $1995^{b}$ & --- & --- & --- & --- \\
\hline $1996^{\mathrm{b}}$ & --- & --- & --- & --- \\
\hline $1997^{\mathrm{b}}$ & --- & --- & --- & --- \\
\hline $1998^{b}$ & --- & --- & --- & --- \\
\hline $1999^{b}$ & --- & --- & --- & --- \\
\hline $2000^{\mathrm{b}}$ & --- & --- & --- & --- \\
\hline $2001^{\mathrm{b}}$ & --- & --- & --- & --- \\
\hline $2002^{b}$ & --- & --- & --- & --- \\
\hline $2003^{b}$ & --- & --- & --- & --- \\
\hline 2004 & --- & --- & --- & --- \\
\hline
\end{tabular}


Table 6b. Continued.

\begin{tabular}{|c|c|c|c|c|}
\hline \multirow[b]{2}{*}{ Year } & \multicolumn{4}{|c|}{ Percentile passage dates at Lower Granite Dam } \\
\hline & 10th & 50th & 90th & Range \\
\hline \multicolumn{5}{|c|}{ Imnaha River (upper) } \\
\hline 1993 & 24 April & 14 May & 28 May & 15 April-23 June \\
\hline 1994 & 24 April & 08 May & 09 June & 20 April-11 Augus \\
\hline 1995 & 13 April & 02 May & 03 June & 10 April-07 July \\
\hline 1996 & 16 April & 26 April & 18 May & 14 April-12 June \\
\hline 1997 & 11 April & 19 April & 11 May & 03 April-02 June \\
\hline 1998 & 11 April & 28 April & 13 May & 03 April-24 May \\
\hline 1999 & 22 April & 08 May & 26 May & 17 April-03 June \\
\hline 2000 & 14 April & 02 May & 24 May & 12 April-16 June \\
\hline 2001 & 21 April & 30 April & 16 May & 08 April-28 May \\
\hline 2002 & 16 April & 04 May & 17 May & 15 April-31 May \\
\hline 2003 & 22 April & 08 May & 26 May & 17 April-31 May \\
\hline 2004 & 19 April & 04 May & 22 May & 18 April-8 June \\
\hline \multicolumn{5}{|c|}{ Lostine River } \\
\hline $1990^{\mathrm{d}}$ & --- & --- & --- & --- \\
\hline 1991 & 29 April & 14 May & 26 May & 20 April-09 July \\
\hline 1992 & 16 April & 30 April & 11 May & 12 April-02 June \\
\hline 1993 & 23 April & 03 May & 17 May & 17 April-01 June \\
\hline 1994 & 22 April & 30 April & 16 May & 19 April-07 June \\
\hline 1995 & 12 April & 02 May & 17 May & 08 April-09 June \\
\hline 1996 & 23 April & 15 May & 07 June & 17 April-19 June \\
\hline 1997 & 17 April & 28 April & 16 May & 09 April-21 May \\
\hline $1998^{\mathrm{b}}$ & --- & --- & --- & --- \\
\hline 1999 & $30 \mathrm{March}$ & 09 May & 27 May & 29 March-29 May \\
\hline 2000 & 13 April & 08 May & 25 May & 13 April-03 June \\
\hline 2001 & 25 April & 09 May & 22 May & 10 April-12 June \\
\hline 2002 & 11 April & 21 April & 13 May & 28 March-29 May \\
\hline 2003 & 13 April & 08 May & 26 May & 11 April-03 June \\
\hline 2004 & 15 April & 04 May & 05 June & 14 April-15 June \\
\hline \multicolumn{5}{|c|}{ Minam River } \\
\hline 1999 & 08 April & 28 April & 25 May & 31 March-02 June \\
\hline 2000 & 15 April & 03 May & 22 May & 10 April-29 May \\
\hline 2001 & 25 April & 07 May & 23 May & 08 April-12 June \\
\hline 2002 & 17 April & 03 May & 20 May & 16 April-31 May \\
\hline 2003 & 17 April & 13 May & 29 May & 13 April-01 June \\
\hline 2004 & 15 April & 28 April & 28 May & 08 April-31 May \\
\hline
\end{tabular}

${ }^{\text {a }}$ Includes fish tagged from summer 1995 through spring 1996.

${ }^{\mathrm{b}}$ No parr were tagged the summer prior to this migration year.

${ }^{\mathrm{c}}$ All fish tagged at traps in fall or spring for this migration year.

${ }^{\mathrm{d}}$ Insufficient numbers detected to estimate timing. 
Migration timing at Lower Granite Dam based on streams with 7 or more years of data indicated that dates of the 10th, 50th, and 90th passage percentile varied between streams (Table 7.) Secesh River fish had a significantly earlier timing at Lower Granite Dam for the 10th percentile passage than fish from all other streams except Lake and Big(lower)/Rush Creeks and Lostine and Imnaha (upper) Rivers $(P<0.05)$. Also, Big Creek (upper) fish had significantly later migration timing at the dam than all the other streams except Loon Creek $(P<0.05)$.

For the 50th percentile passage at Lower Granite Dam, Big(lower)/Rush Creeks had significantly earlier arrival timing than fish from all the other streams except Lake and Herd Creeks and the Secesh River $(P<0.05)$. Fish from Big Creek (upper) had significantly later timing at the dam than fish from all other streams $(P<0.05)$. For the 90th percentile passage at the dam, Big(lower)/Rush Creeks fish had significantly earlier timing than fish from all other streams except Herd, Loon, Marsh, Sulphur, and Elk Creeks, and Imnaha (upper), Lostine, and the Secesh Rivers $(P<0.05)$. Fish from Big Creek (upper) had significantly later timing than fish from all other streams except the South Fork Salmon River, Lake, Catherine, Valley, and Chamberlain/W. F. Chamberlain Creeks $(P<0.05)$.

\section{Comparison with Flows}

We grouped first-time detections (expanded) at Lower Granite Dam of all Idaho and Oregon streams combined and compared their collective timing with river flows during the same periods (Figure 5 and Appendix Table 19). Overall, passage at the dam during 2004 occurred between early-April and mid-July, with the middle 80th percentile passage occurring from 16 April to 26 May (Table 8). The peak passage date was 15 April, which coincided with low flow of $64.2 \mathrm{kcfs}$ (Appendix Table 19).

\section{Environmental Information}

Environmental factors varied by month and between locations (Appendix Tables 23-27), as did the percentage of fish collected and/or detected at adjacent traps (Appendix Figures 1-7). 
Table 7. The 95\% confidence intervals for the percentile passage date ranges and mean passage dates, with standard errors (SE), at Lower Granite Dam for wild spring/summer Chinook salmon smolts from streams in Idaho and Oregon.

\begin{tabular}{|c|c|c|c|c|c|c|c|c|c|c|}
\hline \multirow[b]{3}{*}{$\underline{\text { Stream }}$} & \multicolumn{9}{|c|}{ Percentile passage dates at Lower Granite Dam } & \multirow[b]{3}{*}{$\begin{array}{l}\text { Data } \\
\text { years }\end{array}$} \\
\hline & \multicolumn{3}{|c|}{ 10th } & \multicolumn{3}{|c|}{50 th } & \multicolumn{3}{|c|}{ 90th } & \\
\hline & Mean & $95 \% \mathrm{CI}$ & $\begin{array}{l}\text { SE } \\
\text { (d) }\end{array}$ & Mean & $95 \% \mathrm{CI}$ & $\begin{array}{l}\text { SE } \\
\text { (d) }\end{array}$ & Mean & $95 \% \mathrm{CI}$ & $\begin{array}{l}\text { SE } \\
\text { (d) }\end{array}$ & \\
\hline Secesh River & 14 April & 11-17 April & $(1)$ & 26 April & 23-29 April & (4) & 1 June & 23 May-09 June & (4) & 16 \\
\hline S. Fork Salmon R. & 19 April & 15-23 April & $(2)$ & 10 May & 06-14 May & (2) & 6 June & 2-11 June & (2) & 15 \\
\hline Catherine Creek & 26 April & 23-30 April & $(2)$ & 14 May & 10-17 May & (2) & 4 June & 29 May-9 June & (3) & 14 \\
\hline Imnaha R. (upper) & 17 April & 14-20 April & $(1)$ & 2 May & 28 April-6 May & (2) & 22 May & 17-28 May & (2) & 12 \\
\hline Bear Valley Creek & 22 April & 18-27 April & (2) & 8 May & 5-12 May & (2) & 2 June & 27 May-7 June & (2) & 13 \\
\hline Big Creek (upper) & 1 May & 25 April-7 May & (3) & 21 May & 14-28 May & (3) & 12 June & 1-24 June & (5) & 10 \\
\hline Elk Creek & 20 April & 15-25 April & (2) & 6 May & 2-11 May & (2) & 30 May & 24 May-05 June & (3) & 12 \\
\hline Valley Creek & 25 April & 20-30 April & (2) & 14 May & 9-19 May & (2) & 6 June & 30 May-13 June & (3) & 13 \\
\hline Marsh Creek & 19 April & 16-22 April & (1) & 5 May & 30 April-10 May & (2) & 26 May & 20-31 May & (2) & 11 \\
\hline Lake Creek & 15 April & 11-19 April & (2) & 30 April & 26 April-3 May & (2) & 4 June & 26 May-13 June & (4) & 12 \\
\hline Lostine River & 16 April & 12-21 April & (2) & 4 May & 30 April-8 May & (2) & 22 May & 17-27 May & (2) & 13 \\
\hline Sulphur Creek & 21 April & 14-28 April & (3) & 11 May & 1-20 May & (4) & 29 May & 20 May-7 June & (4) & 8 \\
\hline
\end{tabular}


Table 7 . Continued.

\begin{tabular}{|c|c|c|c|c|c|c|c|c|c|c|}
\hline \multirow[b]{3}{*}{ Stream } & \multicolumn{9}{|c|}{ Percentile passage dates at Lower Granite Dam } & \multirow[b]{3}{*}{$\begin{array}{l}\text { Data } \\
\text { years }\end{array}$} \\
\hline & \multicolumn{3}{|c|}{ 10th } & \multicolumn{3}{|c|}{ 50th } & \multicolumn{3}{|c|}{ 90th } & \\
\hline & Mean & $95 \% \mathrm{CI}$ & $\begin{array}{l}\mathrm{SE} \\
\text { (d) }\end{array}$ & Mean & $95 \% \mathrm{CI}$ & $\begin{array}{l}\text { SE } \\
\text { (d) }\end{array}$ & Mean & $95 \% \mathrm{CI}$ & $\begin{array}{l}\text { SE } \\
\text { (d) }\end{array}$ & \\
\hline Cape Horn Creek & 24 April & 17 April-2 May & (3) & 14 May & 6-21 May & (3) & 4 June & 25 May-14 June & (4) & 8 \\
\hline Big (lower)/Rush Cr. & 18 April & 15-21 April & (1) & 27 April & 25-29 April & (1) & 12 May & 7-17 May & (2) & 8 \\
\hline East Fork Salmon R. & 19 April & 15-24 April & (2) & 1 May & 25 April-07 May & (2) & 18 May & 13-23 May & (2) & 7 \\
\hline Loon Creek & 27 April & 22 April-01 May & (2) & 11 May & 7-15 May & (2) & 23 May & 18-28 May & (2) & 7 \\
\hline Herd Creek & 19 April & 14-24 April & (2) & 29 April & 25 April-3 May & (2) & 15 May & 10-19 May & (2) & 8 \\
\hline Grand Ronde R. (upper) & 1 May & 23 April-10 May & (3) & 24 May & 13 May-04 June & (4) & 12 June & 21 May-3 July & $(8)$ & 5 \\
\hline $\begin{array}{l}\text { Imnaha R. (lower) } \\
\text { Chamberlain./WF }\end{array}$ & 12 April & 5-20 April & (2) & 25 April & 14 April-5 May & (3) & 9 May & 2-15 May & (2) & 4 \\
\hline Chamberlain Cr. & 20 April & 14-26 April & (2) & 5 May & 26 April-14 May & (4) & 6 June & 18 May-25 June & $(8)$ & 7 \\
\hline Camas Creek & 27 April & 21 April-3 May & (2) & 16 May & 9-23 May & (3) & 29 May & 24 May-3 June & (2) & 6 \\
\hline Minam River & 18 April & 11-25 April & (2) & 5 May & 25 April-15 May & (3) & 25 May & 18-31 May & (2) & 4 \\
\hline
\end{tabular}




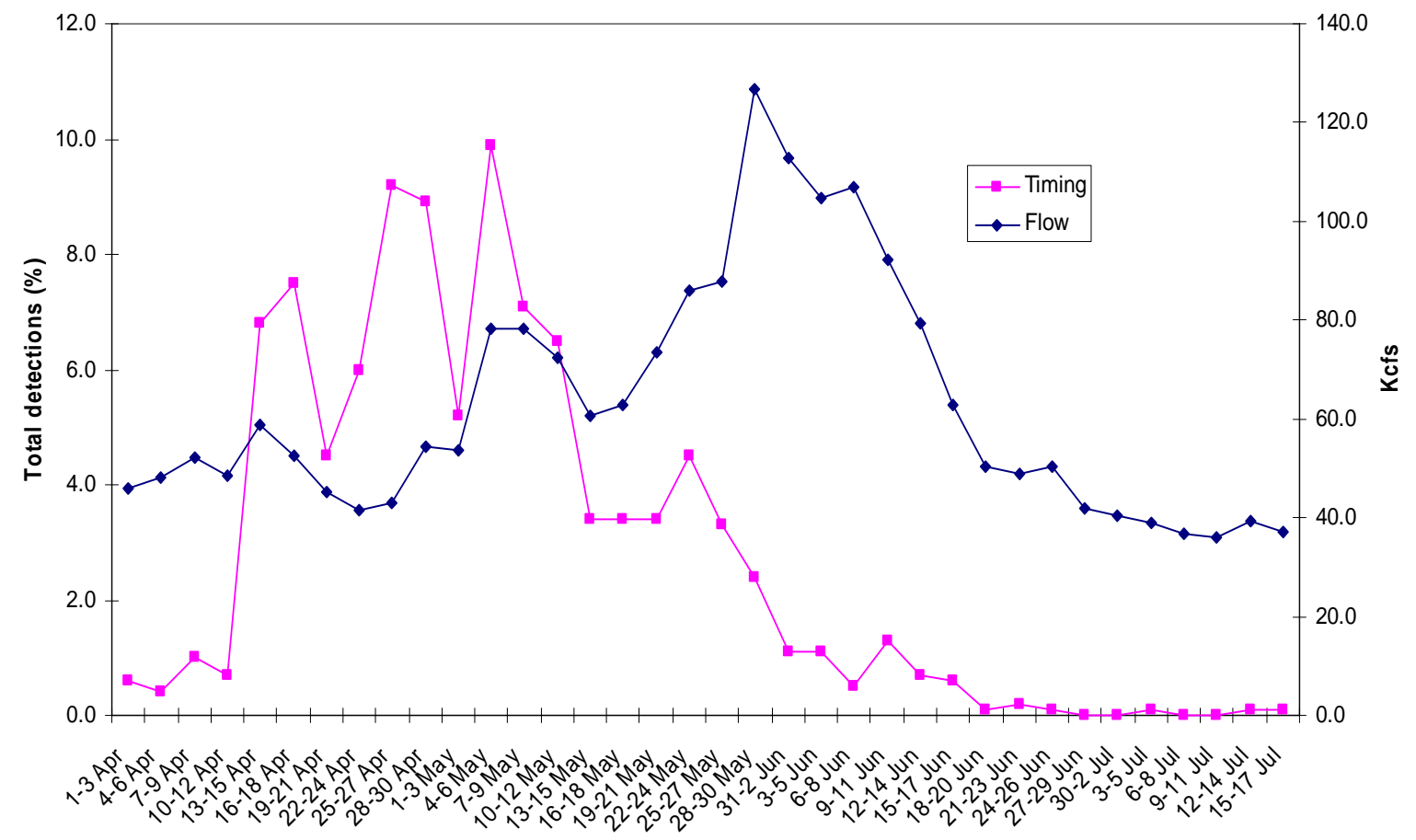

Figure 5. Overall migration timing of PIT-tagged wild spring/summer Chinook salmon smolts with associated river flows at Lower Granite Dam, 2004. Daily detections from 16 Idaho and 4 Oregon streams were pooled in 3-day intervals and expanded based on daily detection probability. River flows at the dam were averaged daily over the same periods. 
Table 8. Accumulated and 2004 passage dates at Lower Granite Dam for combined populations of wild spring/summer Chinook salmon smolts PIT tagged as summer parr in Idaho and Oregon streams.

\begin{tabular}{|c|c|c|c|c|}
\hline \multirow[b]{2}{*}{ Year } & \multicolumn{4}{|c|}{ Passage periods at Lower Granite Dam } \\
\hline & $10 \%$ & $50 \%$ & $90 \%$ & Range \\
\hline $1989^{\mathrm{a}}$ & 23 April & 14 May & 13 June & 04 April-22 July \\
\hline 1990 & 19 April & 07 May & 07 June & 05 April-18 July \\
\hline 1991 & 01 May & 18 May & 12 June & 13 April-20 July \\
\hline 1992 & 15 April & 02 May & 27 May & 05 April-27 July \\
\hline 1993 & 26 April & 14 May & 31 May & 14 April-10 August \\
\hline 1994 & 22 April & 08 May & 01 June & 13 April-04 Sept. \\
\hline 1995 & 17 April & 09 May & 04 June & 08 April-22 Sept. \\
\hline $1996^{\mathrm{a}, \mathrm{b}}$ & 15 April & 27 April & 19 May & 09 April-15 July \\
\hline $1997^{\mathrm{a}, \mathrm{b}}$ & 12 April & 24 April & 18 May & 31 March-22 Sept. \\
\hline $1998^{b}$ & 11 April & 02 May & 23 May & 31 March-07 Aug. \\
\hline 1999 & 20 April & 03 May & 28 May & 27 March-08 July \\
\hline 2000 & 17 April & 07 May & 30 May & 10 April-20 July \\
\hline 2001 & 26 April & 09 May & 27 May & 06 April-07 July \\
\hline 2002 & 16 April & 03 May & 30 May & 28 March-05 July \\
\hline 2003 & 18 April & 11 May & 29 May & 31 March-04 July \\
\hline 2004 & 16 April & 03 May & 26 May & 01 April-16 July \\
\hline
\end{tabular}

${ }^{\text {a }}$ No fish were tagged from the Middle Fork of the Salmon River drainage for this migration year.

${ }^{b}$ This migration year represented by a much higher proportion of fish from Oregon streams than other years. 


\section{DISCUSSION}

Mortality rates associated with collection and tagging in 2003 were comparable to those in earlier years (Achord et al. 1992, 1994, 1995a,b, 1996a,b, 1997, 1998, 2000, 2001a,b, 2002, 2003, 2004).

The in-stream PIT-tag monitoring system used in Valley Creek in 2003-2004, enabled us to calculate survival estimates and migration timing for wild Chinook salmon juveniles leaving this stream. However, only $251(10 \%)$ total juvenile Chinook salmon were detected at these monitors. In order to increase the precision of these estimates, we will either need to increase the antenna size or number, or the tagging sample size.

Results from in-stream monitoring indicated that a high proportion of wild juvenile Chinook salmon moved out of Valley Creek during the winter (32.3\%). This finding has important implications for intensive fish-monitoring studies throughout Idaho because these studies use rotary screw traps, which are inoperable during winter in most areas. Perhaps a combination of rotary screw traps and in-stream PIT-tag monitoring may be appropriate for some locations or studies.

Overall mean growth from the parr to smolt stage was almost identical to that measured in 2003 (Achord et al. 2004). Mean growth in 2004 was less than in 2001, when we observed mean daily growth rates of $0.16 \mathrm{~mm}$ and $0.042 \mathrm{~g}$ for wild fish from many of the same streams (Achord et al. 2002). High densities of parr in these streams during summer 2002 and 2003 may have contributed to these reduced growth rates (especially weight) of wild fish observed in 2004.

For combined Idaho and Oregon steams, annual parr-to-smolt survival estimates over the last 12 years have ranged from 8.1 to $24.4 \%$, with an average annual survival rate of $16.9 \%$. We measured the lowest parr-to-smolt survival estimates in 2003 and 2004 , at 8.8 and $8.1 \%$, respectively. These low estimates may have resulted from the much higher parr density in these years: wild adult returns to the Snake River basin in 2001 and 2002 were more than an order of magnitude greater than returns from 1994 to 1996 when we measured the highest subsequent parr to smolt survival (20.6 to 24.4\%).

In 2004, we again observed that wild fish detected at the dam in April and May had been significantly larger at release than fish migrating after May. This suggests that size is an important factor related to either the initiation of the smolt stage or to other 
life-history dynamics that affect migration timing of wild fish. We also observed that the 50th and 90th passage percentiles at Lower Granite Dam occurred in early and late May, respectively, similar to timing observed in warm years.

In 2004, climate conditions were close to normal, but flows were low during most of the spring. As noted in our previous reports, wild Chinook salmon smolt passage timing at Lower Granite Dam for individual wild populations has been highly variable and usually protracted, with timing patterns for some populations ranging from early to late spring. However, shifts in the passage timing distribution for these populations have been less than 1 to 5 weeks over all years. Complex yearly interrelationships between flow and annual climatic conditions are primary factors contributing to passage timing. However, water temperatures in streams above the dam, turbidity, physiological development, variability in stock behavior, fish size, and other yet unknown factors may all contribute substantially to wild smolt passage timing at the dam.

As additional environmental monitors, in-stream PIT-tag monitors, and traps are installed in study streams, we can more accurately monitor fry, parr, and smolt movements out of rearing areas and examine the relationships between these movements and environmental conditions within the streams. Mapped over time, this information, along with weather and climate data, may provide tools for the prediction of movement in different wild fish stocks. Such tools are vital to recovery planning for threatened or (ESA) endangered species of Pacific salmon. 


\section{ACKNOWLEDGMENTS}

We thank Bonneville Power Administration for funding this project and Douglas Marsh, Gordon Axel, Richard Nelson, William Muir, Richard Zabel, Jeffery Moser, Steve Brewer, Scott Shields, John Ferguson, Regan McNatt, Dan Lyman, Lanesa Hodge, Paul Kemp, and Randy Bunch for their excellent assistance in collecting and PIT tagging fish for this study. Also, thanks to personnel from the Idaho Department of Fish and Game and the Nez Perce Tribe for providing data from trapping operations in natal rearing areas.

We thank the many people and agencies involved with making in-stream PIT-tag monitoring in lower Valley Creek a success from 2002 to 2004. These include William Wassard, Earl Prentice, Richard Nelson, Jeffrey Moser, L. Scott Davidson, and Galen Wolf of National Marine Fisheries Service; Gary Gadwa of the Idaho Department of Fish and Game; Scott Livingston, Darren Chase, Donald Warf, and David Marvin of the Pacific States Marine Fisheries Commission; Toby Tabor and Jerry Lockhart of the Nez Perce Tribe; Douglas Taki of the Shoshone-Bannock Tribe; private landowners George McKovich and Jack See; and numerous other people from local, state, and federal agencies. 


\section{REFERENCES}

Achord, S., G. A. Axel, E. E. Hockersmith, B. P. Sandford, M. B. Eppard, and G. M. Matthews. 2001a. Monitoring the migrations of wild Snake River spring/summer Chinook salmon smolts, 1999. Report of the National Marine Fisheries Service to the Bonneville Power Administration, Portland, Oregon. (www.efw.bpa.gov/cgibin/efw/FW/welcome.cgi).

Achord, S., G. A. Axel, E. E. Hockersmith, B. P. Sandford, M. B. Eppard, and G. M. Matthews. 2001b. Monitoring the migrations of wild Snake River spring/summer Chinook salmon smolts, 2000. Report of the National Marine Fisheries Service to the Bonneville Power Administration, Portland, Oregon. (www.efw.bpa.gov/cgi-bin/efw/FW/welcome.cgi).

Achord, S., G. A. Axel, E. E. Hockersmith, B. P. Sandford, M. B. Eppard, and G. M. Matthews. 2002. Monitoring the migrations of wild Snake River spring/summer Chinook salmon smolts, 2001. Report of the National Marine Fisheries Service to the Bonneville Power Administration, Portland, Oregon. (www.efw.bpa.gov/cgibin/efw/FW/welcome.cgi).

Achord, S., M. B. Eppard, E. E. Hockersmith, B. P. Sandford, G. A. Axel, and G. M. Matthews. 2000. Monitoring the migrations of wild Snake River spring/summer Chinook salmon smolts, 1998. Report of the National Marine Fisheries Service to the Bonneville Power Administration, Portland, Oregon. (www.efw.bpa.gov/cgibin/efw/FW/welcome.cgi).

Achord, S., M. B. Eppard, E. E. Hockersmith, B. P. Sandford, and G. M. Matthews. 1997. Monitoring the migrations of wild Snake River spring/summer Chinook salmon smolts, 1996. Report of the National Marine Fisheries Service to the Bonneville Power Administration, Portland, Oregon. (www.efw.bpa.gov/cgibin/efw/FW/welcome.cgi).

Achord, S., M. B. Eppard, E. E. Hockersmith, B. P. Sandford, and G. M. Matthews. 1998. Monitoring the migrations of wild Snake River spring/summer Chinook salmon smolts, 1997. Report of the National Marine Fisheries Service to the Bonneville Power Administration, Portland, Oregon. (www.efw.bpa.gov/cgibin/efw/FW/welcome.cgi). 
Achord, S., M. B. Eppard, B. P. Sandford, and G. M. Matthews. 1996a. Monitoring the migrations of wild Snake River spring/summer Chinook salmon smolts, 1995. Report of the National Marine Fisheries Service to the Bonneville Power Administration, Portland, Oregon. (www.efw.bpa.gov/cgibin/efw/FW/welcome.cgi).

Achord, S., J. R. Harmon, D. M. Marsh, B. P. Sandford, K. W. McIntyre, K. L. Thomas, N. N. Paasch, and G. M. Matthews. 1992. Research related to transportation of juvenile salmonids on the Columbia and Snake Rivers, 1991. Report of the National Marine Fisheries Service to the U.S. Army Corps of Engineers, Walla Walla, Washington.

Achord, S., E. E. Hockersmith, B. P. Sandford, R. A. McNatt, B. E. Feist, and G. M. Matthews. 2003. Monitoring the migrations of wild Snake River spring/summer Chinook salmon smolts, 2002. Report of the National Marine Fisheries Service to the Bonneville Power Administration, Portland, Oregon. (www.efw.bpa.gov/cgibin/efw/FW/welcome.cgi).

Achord, S., D. J. Kamikawa, B. P. Sandford, and G. M. Matthews. 1995a. Monitoring the migrations of wild Snake River spring/summer Chinook salmon smolts, 1993. Report of the National Marine Fisheries Service to the Bonneville Power Administration, Portland, Oregon. (www.efw.bpa.gov/cgibin/efw/FW/welcome.cgi).

Achord, S., D. J. Kamikawa, B. P. Sandford, and G. M. Matthews. 1995b. Monitoring the migrations of wild Snake River spring/summer Chinook salmon smolts, 1994. Report of the National Marine Fisheries Service to the Bonneville Power Administration, Portland, Oregon. (www.efw.bpa.gov/cgibin/efw/FW/welcome.cgi).

Achord, S., G. M. Matthews, O. W. Johnson, and D. M. Marsh. 1996b. Use of Passive Integrated Transponder (PIT) tags to monitor migration timing of Snake River Chinook salmon smolts. North American Journal of Fisheries Management 16:302-313.

Achord, S., G. M. Matthews, D. M. Marsh, B. P. Sandford, and D. J. Kamikawa. 1994. Monitoring the migrations of wild Snake River spring and summer Chinook salmon smolts, 1992. Report of the National Marine Fisheries Service to the Bonneville Power Administration, Portland, Oregon. (www.efw.bpa.gov/cgibin/efw/FW/welcome.cgi). 
Achord, S., R. A. McNatt, E. E. Hockersmith, B. P. Sandford, K. W. McIntyre, N. N.

Paasch, J. G. Williams, and G. M. Matthews. 2004. Monitoring the migrations of wild Snake River spring/summer Chinook salmon smolts, 2003. Report of the National Marine Fisheries Service to the Bonneville Power Administration, Portland, Oregon. (www.efw.bpa.gov/cgi-bin/efw/FW/welcome.cgi).

Downing, S. L., E. F. Prentice, B. W. Peterson, E. P. Nunnallee, and B. F. Jonasson. 2001. Development and evaluation of passive integrated transponder tag technology, annual report: 1999 to 2000. Report of the National Marine Fisheries Service to the Bonneville Power Administration, Portland, Oregon. (www.efw.bpa.gov/cgi-bin/efw/FW/welcome.cgi).

Downing, S. L., E. F. Prentice, E. P. Nunnallee, and B. F. Jonasson. 2004. Development and evaluation of passive integrated transponder tag technology, 2000-2002. Report of the National Marine Fisheries Service to the Bonneville Power Administration, Portland, Oregon. (www.efw.bpa.gov/cgibin/efw/FW/welcome.cgi).

Efron, B., and R. J. Tibshirani. 1993. An introduction to the bootstrap. Chapman and Hall, Norwell, MA, 436 p.

Ledgerwood, R. D., B. A. Ryan, E. M. Dawley, E. P. Nunnallee, and J. W. Ferguson. 2004. A surface trawl to detect migrating juvenile salmonids tagged with passive integrated transponder tags. North American Journal of Fisheries Management $24: 440-451$.

Matthews, G. M., J. R. Harmon, S. Achord, O. W. Johnson, and L. A. Kubin. 1990. Evaluation of transportation of juvenile salmonids and related research on the Snake and Columbia Rivers, 1989. Report of the National Marine Fisheries Service to the U.S. Army Corps of Engineers, Northwestern Division, Walla Walla District.

PSMFC (Pacific States Marine Fisheries Commission). 1996. The Columbia Basin PIT Tag Information System (PTAGIS). PSMFC, Portland, Oregon. Online database available www.psmfc.org.pittag/ (accessed October 2004).

Petersen, R. G. 1985. Design and analysis of experiments. Marcel Dekker, New York, $429 \mathrm{p}$. 
Prentice, E. F., T. A. Flagg, and C. S. McCutcheon. 1990. PIT-tag monitoring systems for hydroelectric dams and fish hatcheries. American Fisheries Society Symposium 7:323-334.

Sandford, B. P., and S. G. Smith. 2002. Estimation of smolt-to-adult return percentages for Snake River Basin anadromous salmonids, 1990-1997. Journal of Agricultural, Biological, and Environmental Statistics 7(2):243-263. 


\section{APPENDIX}

Data Tables and Figures 
Appendix Table 1. Summary of tagging dates, numbers collected, tagged, released, and minimum, maximum, and mean lengths and weights of wild Chinook salmon parr, collected and PIT tagged in various Idaho streams, 2003.

\begin{tabular}{|c|c|c|c|c|c|c|c|c|c|c|c|}
\hline & \multicolumn{3}{|c|}{ Number of fish } & \multirow{2}{*}{\multicolumn{4}{|c|}{ Collected }} & \multirow{2}{*}{\multicolumn{4}{|c|}{ Released }} \\
\hline & \multirow[b]{3}{*}{ Collected } & \multirow[b]{3}{*}{ Tagged } & \multirow{3}{*}{$\begin{array}{l}\text { Tagged } \\
\text { and } \\
\text { released }\end{array}$} & & & & & & & & \\
\hline & & & & \multicolumn{2}{|c|}{ Length (mm) } & \multicolumn{2}{|c|}{ Weight (g) } & \multicolumn{2}{|c|}{ Length (mm) } & \multicolumn{2}{|c|}{ Weight (g) } \\
\hline & & & & Range & Mean & Range & Mean & Range & Mean & Range & Mean \\
\hline \multicolumn{12}{|c|}{ Bear Valley Creek } \\
\hline 22-24 July & 1,995 & 1,496 & 1,494 & $44-123$ & 59.1 & $0.8-7.8$ & 2.8 & $50-85$ & 61.4 & $1.0-7.8$ & 3.0 \\
\hline \multicolumn{12}{|l|}{ Elk Creek } \\
\hline 25-29 July & 1,819 & 1,522 & 1,520 & $44-81$ & 61.4 & $0.7-8.3$ & 3.1 & $52-81$ & 62.9 & $1.7-8.3$ & 3.3 \\
\hline \multicolumn{12}{|c|}{ Marsh Creek } \\
\hline \multicolumn{12}{|c|}{30 July; 1} \\
\hline Aug & 1,812 & 1,535 & 1,535 & $41-86$ & 63.3 & $0.9-8.1$ & 3.5 & $51-86$ & 64.6 & $1.8-8.1$ & 3.7 \\
\hline \multicolumn{12}{|c|}{ Sulphur Creek } \\
\hline 31-Jul & 1,319 & 1,053 & 1,048 & $42-122$ & 62.1 & $1.7-5.2$ & 3.0 & $43-93$ & 62.9 & $2.0-5.2$ & 3.1 \\
\hline \multicolumn{12}{|c|}{ Cape Horn Creek } \\
\hline 01-02 Aug & 2,319 & 671 & 671 & $37-118$ & 53.8 & $0.6-17.8$ & 2.8 & $49-94$ & 61.4 & $1.4-11.4$ & 3.4 \\
\hline \multicolumn{12}{|l|}{ Valley Creek } \\
\hline 04-06 Aug & 3,152 & 2,504 & 2,498 & $43-167$ & 61.1 & $0.9-23.5$ & 3.4 & $50-167$ & 62.8 & $1.5-8.9$ & 3.5 \\
\hline \multicolumn{12}{|l|}{ Loon Creek } \\
\hline 08-Aug & 952 & 861 & 860 & $46-85$ & 63.4 & $1.2-7.7$ & 3.6 & $52-85$ & 64.5 & $2.0-7.7$ & 3.7 \\
\hline \multicolumn{12}{|c|}{ Camas Creek } \\
\hline 08-Aug & 1,201 & 1,007 & 1,005 & $44-116$ & 60.5 & $1.1-7.5$ & 3.2 & $50-84$ & 62.0 & $1.7-7.5$ & 3.3 \\
\hline \multicolumn{12}{|l|}{ Herd Creek } \\
\hline 11-Aug & 1,047 & 968 & 968 & $44-184$ & 66.7 & $1.3-7.9$ & 3.9 & $52-184$ & 67.3 & $1.6-7.9$ & 4.1 \\
\hline \multicolumn{12}{|c|}{ Big Creek (upper) } \\
\hline 15-16 Aug & 1,933 & 1,504 & 1,504 & $43-110$ & 59.9 & $1.0-15.4$ & 3.3 & $50-90$ & 61.7 & $1.7-7.4$ & 3.4 \\
\hline \multicolumn{12}{|c|}{ Big Creek (lower) } \\
\hline 21-Aug & 943 & 899 & 899 & $53-105$ & 71.9 & $1.6-14$ & 4.4 & $55-105$ & 72.1 & $1.5-14$ & 4.4 \\
\hline \multicolumn{12}{|l|}{ Rush Creek } \\
\hline 22-Aug & 59 & 52 & 52 & $52-96$ & 71.7 & --- & --- & $61-84$ & 71.8 & --- & --- \\
\hline West Fork & Chamberla & ain Cree & & & & & & & & & \\
\hline 21-Aug & 997 & 756 & 753 & $42-110$ & 61.0 & $1.7-13.9$ & 3.4 & $52-92$ & 63.3 & $1.7-6.6$ & 3.2 \\
\hline Chamberla & in Creek & & & & & & & & & & \\
\hline 22-Aug & 316 & 243 & 243 & $41-93$ & 57.9 & $1.1-10.6$ & 2.7 & $51-82$ & 59.4 & $1.5-4.4$ & 2.8 \\
\hline South Fork & Salmon $\mathbf{R}$ & River & & & & & & & & & \\
\hline 25-26 Aug & 2,608 & 1,490 & 1,490 & $38-138$ & 56.9 & $0.7-10.7$ & 3.1 & $50-138$ & 62.3 & $1.5-9.2$ & 3.2 \\
\hline Secesh Rive & & & & & & & & & & & \\
\hline 28-29 Aug & 1,708 & 1,146 & 1,142 & $39-97$ & 58.1 & $0.7-10.5$ & 2.7 & $48-97$ & 61.9 & $1.3-9.4$ & 3.0 \\
\hline Lake Creek & & & & & & & & & & & \\
\hline 30-Aug & 1,029 & 664 & 664 & $36-296$ & 58.0 & $0.4-11.2$ & 3.1 & $50-103$ & 61.5 & $1.2-11.2$ & 3.2 \\
\hline Total/mean & 25,209 & 18,371 & 18,346 & $36-296$ & 60.4 & $0.4-23.5$ & 3.2 & $43-184$ & 63.2 & $1.0-11.4$ & 3.4 \\
\hline
\end{tabular}


Appendix Table 2a. Summary of tagging dates, start tagging times and temperatures $\left({ }^{\circ} \mathrm{C}\right)$, release dates, times, and temperatures, methods of capture, distance (in kilometers) from the mouth of the stream to the release point, number released (in 2003), and number/percent of first-time detections (unadjusted) for each tag group at six downstream dams and the PIT-tag trawl at the mouth of the Columbia River during 2004.

\begin{tabular}{|c|c|c|c|c|c|c|c|c|c|c|c|}
\hline & \multicolumn{4}{|c|}{ Tagging } & \multicolumn{5}{|c|}{ Release } & \multicolumn{2}{|c|}{ Detection } \\
\hline & Date & $\begin{array}{l}\text { Time } \\
\text { (PST) }\end{array}$ & $\begin{array}{c}\text { Temp } \\
\left({ }^{\circ} \mathrm{C}\right)\end{array}$ & Capture method & Date & $\begin{array}{l}\text { Time } \\
\text { (PST) }\end{array}$ & $\begin{array}{l}\text { Temp. } \\
\left({ }^{\circ} \mathrm{C}\right)\end{array}$ & $\begin{array}{c}\text { River } \\
\text { km }\end{array}$ & $\mathrm{n}$ & $\mathrm{n}$ & $(\%)$ \\
\hline \multicolumn{12}{|c|}{ Bear Valley Creek } \\
\hline SA03203.BV1 & $22 \mathrm{Jul}$ & 0543 & 14.0 & Beach seine & $23 \mathrm{Jul}$ & 0400 & 14.0 & 9 & 127 & 7 & 5.5 \\
\hline SA03203.BV2 & $22 \mathrm{Jul}$ & 0711 & 15.0 & Beach seine & $22 \mathrm{Jul}$ & 0940 & 15.0 & 13 & 540 & 36 & 6.7 \\
\hline SA03204.BV1 & $23 \mathrm{Jul}$ & 0704 & 15.0 & Beach seine & $23 \mathrm{Jul}$ & 0945 & 16.0 & 13 & 509 & 24 & 4.7 \\
\hline SA03205.BV1 & $24 \mathrm{Jul}$ & 0600 & 14.0 & Beach seine & $25 \mathrm{Jul}$ & 0415 & 14.0 & 15 & 318 & 23 & 7.3 \\
\hline \multicolumn{12}{|l|}{ Elk Creek } \\
\hline SA03206.EC1 & $25 \mathrm{Jul}$ & 0615 & 14.0 & Beach seine & $26 \mathrm{Jul}$ & 0450 & 14.0 & 0 & 153 & 12 & 7.8 \\
\hline SA03206.EC2 & $25 \mathrm{Jul}$ & 0915 & 14.0 & Beach seine & $25 \mathrm{Jul}$ & 0915 & 15.5 & 2 & 571 & 46 & 8.0 \\
\hline SA03209.EC1 & $28 \mathrm{Jul}$ & 0532 & 13.0 & Beach seine & $28 \mathrm{Jul}$ & 0930 & 14.5 & 3 & 102 & 05 & 4.9 \\
\hline SA03209.EC2 & $28 \mathrm{Jul}$ & 0919 & 14.5 & Shock & $28 \mathrm{Jul}$ & 1200 & 15.5 & 2 & 303 & 14 & 4.6 \\
\hline SA03210.EC1 & $29 \mathrm{Jul}$ & 0628 & 13.5 & Shock & $29 \mathrm{Jul}$ & 0815 & 14.0 & 2 & 391 & 33 & 8.4 \\
\hline \multicolumn{12}{|l|}{ Marsh Creek } \\
\hline SA03211.MC1 & $30 \mathrm{Jul}$ & 0744 & 10.0 & Beach seine & $31 \mathrm{Jul}$ & 0520 & 10.0 & 12 & 132 & 06 & 4.5 \\
\hline SA03211.MC2 & $30 \mathrm{Jul}$ & 0912 & 13.0 & Shock & $30 \mathrm{Jul}$ & 1130 & 16.0 & 12 & 386 & 37 & 9.6 \\
\hline SA03212.MC1 & $31 \mathrm{Jul}$ & 0513 & 10.0 & Shock & $31 \mathrm{Jul}$ & 0700 & 10.0 & 14 & 164 & 15 & 9.1 \\
\hline SA03212.MC2 & $31 \mathrm{Jul}$ & 0647 & 10.0 & Shock & $31 \mathrm{Jul}$ & 1130 & 16.0 & 14 & 665 & 47 & 7.1 \\
\hline SA03213.MC1 & 1 Aug & 0536 & 09.0 & Shock & 1Aug & 0700 & 09.0 & 2 & 100 & 07 & 7.0 \\
\hline SA03213.MC2 & 1 Aug & 0630 & 09.0 & Shock & 1Aug & 0700 & 09.0 & 2 & 88 & 03 & 3.4 \\
\hline
\end{tabular}


Appendix Table 2a. Continued.

\begin{tabular}{|c|c|c|c|c|c|c|c|c|c|c|c|}
\hline & \multicolumn{4}{|c|}{ Tagging } & \multicolumn{5}{|c|}{ Release } & \multicolumn{2}{|c|}{ Detection } \\
\hline & Date & $\begin{array}{l}\text { Time } \\
\text { (PST) }\end{array}$ & $\begin{array}{c}\text { Temp } \\
\left({ }^{\circ} \mathrm{C}\right)\end{array}$ & Capture method & Date & $\begin{array}{l}\text { Time } \\
\text { (PST) }\end{array}$ & $\begin{array}{c}\text { Temp. } \\
\left({ }^{\circ} \mathrm{C}\right)\end{array}$ & $\begin{array}{c}\text { River } \\
\text { km }\end{array}$ & $\mathrm{n}$ & $\mathrm{n}$ & $(\%)$ \\
\hline \multicolumn{12}{|c|}{ Cape Horn Creek } \\
\hline SA03213.CH1 & $1 \mathrm{Aug}$ & 0700 & 09.5 & Shock & $2 \mathrm{Aug}$ & 0600 & 11.0 & 2 & 122 & 7 & 5.6 \\
\hline SA03213.CH2 & $1 \mathrm{Aug}$ & 0811 & 11.0 & Shock & $1 \mathrm{Aug}$ & 1045 & 12.0 & 2 & 328 & 16 & 4.9 \\
\hline SA03214.CH1 & $2 \mathrm{Aug}$ & 0431 & 08.5 & Shock & 2 Aug & 0500 & 08.5 & 6 & 74 & 2 & 2.7 \\
\hline SA03214.CH2 & 2 Aug & 0641 & 08.5 & Shock & 2 Aug & 1000 & 11.0 & 6 & 147 & 12 & 8.2 \\
\hline \multicolumn{12}{|l|}{ Sulphur Creek } \\
\hline SA03212.SU1 & $31 \mathrm{Jul}$ & 0823 & 11.0 & Beach seine & 1 Aug & 0730 & 11.0 & 5 & 183 & 4 & 2.2 \\
\hline SA03212.SU2 & $31 \mathrm{Jul}$ & 0956 & 13.0 & Beach seine & $31 \mathrm{Jul}$ & 1230 & 16.5 & 5 & 865 & 34 & 3.9 \\
\hline \multicolumn{12}{|l|}{ Valley Creek } \\
\hline SA03216.VC1 & 4 Aug & 0600 & 12.5 & Beach seine & 5 Aug & 0430 & 13.0 & 4 & 96 & 1 & 1.0 \\
\hline SA03216.VC2 & 4 Aug & 0641 & 12.5 & Beach seine & 4 Aug & 1100 & 17.0 & 7 & 1,035 & 34 & 3.3 \\
\hline SA03217.VC1 & 5 Aug & 0735 & 14.0 & Shock & 5 Aug & 1145 & 20.0 & 7 & 746 & 41 & 5.5 \\
\hline SA03218.VC1 & 6 Aug & 0605 & 10.5 & Shock & 6 Aug & 1000 & 13.5 & 18 & 621 & 49 & 7.9 \\
\hline \multicolumn{12}{|l|}{ Loon Creek } \\
\hline SA03220.LN1 & 8 Aug & 0616 & 09.5 & Shock & 9 Aug & 0500 & 09.5 & 36 & 98 & 11 & 11.2 \\
\hline SA03220.LN2 & 8 Aug & 0724 & 11.0 & Shock & 8 Aug & 1215 & 13.0 & 35 & 715 & 99 & 13.8 \\
\hline SA03220.LN3 & 8 Aug & 1238 & 14.0 & Shock & 8 Aug & 1330 & 15.5 & 36 & 47 & 3 & 6.4 \\
\hline
\end{tabular}


Appendix Table 2a. Continued.

\begin{tabular}{|c|c|c|c|c|c|c|c|c|c|c|c|}
\hline & \multicolumn{4}{|c|}{ Tagging } & \multicolumn{5}{|c|}{ Release } & \multicolumn{2}{|c|}{ Detection } \\
\hline & Date & $\begin{array}{l}\text { Time } \\
\text { (PST) }\end{array}$ & Temp $\left({ }^{\circ} \mathrm{C}\right)$ & Capture method & Date & $\begin{array}{l}\text { Time } \\
\text { (PST) }\end{array}$ & $\begin{array}{c}\text { Temp. } \\
\left({ }^{\circ} \mathrm{C}\right)\end{array}$ & $\begin{array}{l}\text { River } \\
\mathrm{km}\end{array}$ & $\mathrm{n}$ & $\mathrm{n}$ & $(\%)$ \\
\hline \multicolumn{12}{|l|}{ Camas Creek } \\
\hline SA03220.CA1 & 8 Aug & 0830 & 10.5 & Shock & 9 Aug & 0800 & 10.0 & 23 & 149 & 17 & 11.4 \\
\hline SA03220.CA2 & 8 Aug & 0952 & 11.0 & Shock & 8 Aug & 1400 & 16.0 & 23 & 856 & 84 & 9.8 \\
\hline \multicolumn{12}{|l|}{ Herd Creek } \\
\hline SA03223.HC1 & 11 Aug & 0648 & 09.5 & Shock & 12 Aug & 0800 & 11.0 & 2 & 129 & 18 & 14.0 \\
\hline SA03223.HC2 & 11 Aug & 0753 & 11.0 & Shock & 11 Aug & 1200 & 17.0 & 2 & 839 & 97 & 11.6 \\
\hline \multicolumn{12}{|c|}{ Big Creek (upper) } \\
\hline SA03227.BC1 & 15 Aug & 0550 & 08.5 & Shock & 16 Aug & 0500 & 09.0 & 56 & 136 & 13 & 9.6 \\
\hline SA03227.BC2 & 15 Aug & 0709 & 09.0 & Shock & $15 \mathrm{Aug}$ & 1330 & 13.0 & 56 & 928 & 70 & 7.5 \\
\hline SA03228.BC1 & 16 Aug & 0543 & 07.5 & Shock & 16 Aug & 0900 & 11.0 & 57 & 440 & 35 & 8.0 \\
\hline \multicolumn{12}{|c|}{ Big Creek (lower) } \\
\hline SA03233.LB1 & 21 Aug & 1010 & 14.5 & Shock & 22 Aug & 0645 & 14.5 & 9 & 109 & 12 & 11.1 \\
\hline SA03233.LB2 & $21 \mathrm{Aug}$ & 1106 & 14.5 & Shock & 21 Aug & 1530 & 15.5 & 10 & 790 & 128 & 16.2 \\
\hline \multicolumn{12}{|l|}{ Rush Creek } \\
\hline SA03234.RC1 & 22 Aug & 0733 & 14.5 & Shock & 22 Aug & 0930 & 15.0 & 1 & 52 & 03 & 5.9 \\
\hline \multicolumn{12}{|c|}{ W.F. Chamberlain Creek } \\
\hline SA03233.WC1 & 21 Aug & 0844 & 09.0 & Beach seine & 22 Aug & 1300 & 13.0 & 2 & 120 & 11 & 9.2 \\
\hline SA03233.WC2 & 21Aug & 0937 & 10.0 & Beach seine & 21 Aug & 1230 & 13.5 & 2 & 633 & 36 & 5.7 \\
\hline
\end{tabular}


Appendix Table 2a. Continued.

\begin{tabular}{|c|c|c|c|c|c|c|c|c|c|c|c|}
\hline & \multicolumn{4}{|c|}{ Tagging } & \multicolumn{5}{|c|}{ Release } & \multicolumn{2}{|c|}{ Detection } \\
\hline & Date & $\begin{array}{l}\text { Time } \\
(\mathrm{PST})\end{array}$ & $\begin{array}{l}\text { Temp } \\
\left({ }^{\circ} \mathrm{C}\right)\end{array}$ & Capture method & Date & $\begin{array}{l}\text { Time } \\
\text { (PST) }\end{array}$ & $\begin{array}{l}\text { Temp. } \\
\left({ }^{\circ} \mathrm{C}\right)\end{array}$ & $\begin{array}{c}\text { River } \\
\mathrm{km}\end{array}$ & $\mathrm{n}$ & $\mathrm{n}$ & $(\%)$ \\
\hline \multicolumn{12}{|c|}{ Chamberlain Creek } \\
\hline SA03234.CB1 & 22 Aug & 0707 & 13.0 & Shock & 22 Aug & 1130 & 13.0 & 26 & 243 & 14 & 5.8 \\
\hline \multicolumn{12}{|c|}{ South Fork Salmon River } \\
\hline SA03237.SF1 & 25 Aug & 0641 & 10.5 & Shock & 26 Aug & 0500 & 11.0 & 118 & 91 & 06 & 6.6 \\
\hline SA03237.SF2 & 25 Aug & 0755 & 11.0 & Shock & $25 \mathrm{Aug}$ & 1230 & 15.5 & 118 & 591 & 42 & 7.1 \\
\hline SA03238.SF1 & 26 Aug & 0626 & 09.0 & Shock & 26Aug & 1300 & 14.5 & 123 & 808 & 50 & 6.2 \\
\hline \multicolumn{12}{|l|}{ Secesh River } \\
\hline SA03240.SE1 & 28 Aug & 0643 & 10.5 & Shock & 29 Aug & 0525 & 09.5 & 27 & 88 & 04 & 4.5 \\
\hline SA03240.SE2 & 28 Aug & 0724 & 11.0 & Shock & 28 Aug & 1130 & 14.5 & 27 & 348 & 12 & 3.4 \\
\hline SA03241.SE1 & 29 Aug & 0553 & 09.5 & Shock & 29 Aug & 1215 & 15.0 & 29 & 706 & 30 & 4.2 \\
\hline \multicolumn{12}{|l|}{ Lake Creek } \\
\hline SA03242.LC1 & 30 Aug & 0600 & 07.5 & Shock & 31 Aug & 0620 & 07.0 & 2 & 103 & 10 & 9.6 \\
\hline SA03242.LC2 & 30 Aug & 0706 & 07.5 & Shock & 30 Aug & 1200 & 12.5 & 2 & 561 & 23 & 4.1 \\
\hline
\end{tabular}


Appendix Table 2b. Universal Transverse Mercator grid coordinates of Global Positioning System that identify sampling areas at the beginning and end of daily collections in streams for each collection crew in 2003. Hand-held Garmin GPS III-plus units were used.

\begin{tabular}{|c|c|c|c|c|c|}
\hline \multirow[b]{2}{*}{ Date } & \multirow{2}{*}{$\begin{array}{l}\text { Section } \\
\text { covered }\end{array}$} & \multicolumn{2}{|c|}{ UTM start } & \multicolumn{2}{|c|}{ UTM end } \\
\hline & & Northing & Easting & Northing & Easting \\
\hline \multicolumn{6}{|c|}{ Bear Valley Creek } \\
\hline $7 / 22 / 03$ & Entire stream & 4920697 & $11 \mathrm{TO} 633071$ & --- & 11TO632881 \\
\hline $7 / 23 / 03$ & Entire stream & 4919095 & $11 \mathrm{TO} 630221$ & 4918946 & $11 \mathrm{TO} 629970$ \\
\hline $7 / 24 / 03$ & Entire stream & 4918758 & $11 \mathrm{TO} 629591$ & 4918613 & $11 \mathrm{TO} 629674$ \\
\hline \multicolumn{6}{|c|}{ Elk Creek } \\
\hline $7 / 25 / 03$ & Entire stream & 4918770 & $11 \mathrm{TO} 629542$ & 4918806 & $11 \mathrm{TO} 629470$ \\
\hline $7 / 28 / 03$ & Entire stream & 4918564 & 11 TO629168 & 4918749 & $11 \mathrm{TO} 628837$ \\
\hline $7 / 28 / 03$ & Left bank & 4919079 & $11 \mathrm{TO} 628628$ & 4919303 & $11 \mathrm{TO} 628336$ \\
\hline $7 / 28 / 03$ & Right bank & 4919079 & $11 \mathrm{TO} 628628$ & 4919303 & $11 \mathrm{TO} 628336$ \\
\hline $7 / 29 / 03$ & Right bank & 4919348 & 11TO628101 & 4919425 & $11 \mathrm{TO} 628076$ \\
\hline $7 / 29 / 03$ & Left bank & 4919348 & $11 \mathrm{TO} 628101$ & 4919425 & $11 \mathrm{TO} 628076$ \\
\hline \multicolumn{6}{|c|}{ Marsh Creek } \\
\hline $7 / 30 / 03$ & Entire stream & 4917020 & $11 \mathrm{TO} 646040$ & 4916230 & $11 \mathrm{TO} 646870$ \\
\hline $7 / 30 / 03$ & Entire stream & 4917020 & $11 \mathrm{TO} 646040$ & 4916230 & $11 \mathrm{TO} 646870$ \\
\hline $7 / 31 / 03$ & Entire stream & 4916230 & 11 TO646870 & 4915590 & $11 \mathrm{TO} 647370$ \\
\hline $7 / 31 / 03$ & Entire stream & 4916230 & $11 \mathrm{TO} 646870$ & 4915590 & $11 \mathrm{TO} 647370$ \\
\hline 8/1/03 & Right bank & --- & --- & --- & --- \\
\hline \multicolumn{6}{|c|}{ Sulphur Creek } \\
\hline $7 / 31 / 03$ & Entire stream & 4933054 & 11 TO630949 & 4932717 & $11 \mathrm{TO} 630576$ \\
\hline \multicolumn{6}{|c|}{ Cape Horn Cr } \\
\hline $8 / 1 / 03$ & Left bank & 4917130 & $11 \mathrm{TO} 645830$ & 4916290 & 11TO645380 \\
\hline $8 / 1 / 03$ & Right bank & 4917130 & $11 \mathrm{TO} 645830$ & 4916290 & $11 \mathrm{TO} 645380$ \\
\hline $8 / 2 / 03$ & Left bank & 4913580 & $11 \mathrm{TO} 643560$ & 4912960 & $11 \mathrm{TO} 643070$ \\
\hline $8 / 2 / 03$ & Right bank & 4913580 & $11 \mathrm{TO} 643560$ & 4912960 & $11 \mathrm{TO} 643070$ \\
\hline \multicolumn{6}{|c|}{ Valley Creek } \\
\hline $8 / 4 / 03$ & Entire stream & 4899599 & 11 TO661066 & 4899799 & 11TO660665 \\
\hline $8 / 5 / 03$ & Left bank & 4900600 & $11 \mathrm{TO} 659703$ & 4900696 & 11TO659530 \\
\hline $8 / 5 / 03$ & Right bank & 4900618 & 11 TO659709 & 4901582 & 11TO659426 \\
\hline $8 / 6 / 03$ & Entire stream & 4906193 & $11 \mathrm{TO} 657788$ & 4906397 & 11TO657599 \\
\hline $8 / 6 / 03$ & Entire stream & 4906316 & $11 \mathrm{TO657667}$ & 4906096 & $11 \mathrm{TO} 657273$ \\
\hline
\end{tabular}


Appendix Table 2b. Continued.

\begin{tabular}{|c|c|c|c|c|c|}
\hline \multirow[b]{2}{*}{ Date } & \multirow{2}{*}{$\begin{array}{l}\text { Section } \\
\text { covered }\end{array}$} & \multicolumn{2}{|c|}{ UTM start } & \multicolumn{2}{|c|}{ UTM end } \\
\hline & & Northing & Easting & Northing & Easting \\
\hline \multicolumn{6}{|c|}{ Camas Creek } \\
\hline 8/8/03 & Right bank & 4968467 & $11 \mathrm{TO} 696386$ & 4967684 & $11 \mathrm{TO697017}$ \\
\hline 8/8/03 & Left bank & 4968467 & $11 \mathrm{TO} 696386$ & 4967684 & $11 \mathrm{TO} 697017$ \\
\hline \multicolumn{6}{|c|}{ Loon Creek } \\
\hline 8/8/03 & Right bank & 4942058 & $11 \mathrm{TO} 674895$ & 4939176 & $11 \mathrm{TO} 672270$ \\
\hline 8/8/03 & Right bank & 4942058 & $11 \mathrm{TO} 674895$ & 4941027 & $11 \mathrm{TO} 674062$ \\
\hline \multicolumn{6}{|c|}{ Herd Creek } \\
\hline $8 / 11 / 03$ & Entire stream & 4891761 & $11 \mathrm{TO} 716670$ & 4891651 & $11 \mathrm{TO} 716794$ \\
\hline $8 / 11 / 03$ & Right bank & 4892105 & $11 \mathrm{TO} 716233$ & 4891875 & $11 \mathrm{TO} 716488$ \\
\hline \multicolumn{6}{|c|}{ Big Creek (upper) } \\
\hline $8 / 15 / 03$ & Left bank & 4996670 & $11 \mathrm{TO} 631579$ & 4995266 & $11 \mathrm{TO} 631347$ \\
\hline $8 / 15 / 03$ & Right bank & 4996670 & $11 \mathrm{TO} 631579$ & 4995266 & $11 \mathrm{TO} 631347$ \\
\hline $8 / 16 / 03$ & Left bank & 4994426 & $11 \mathrm{TO} 630823$ & 4994016 & $11 \mathrm{TO} 630700$ \\
\hline $8 / 16 / 03$ & Right bank & 4994426 & $11 \mathrm{TO} 630823$ & 4994016 & $11 \mathrm{TO} 630700$ \\
\hline \multicolumn{6}{|c|}{ Big Creek (lower) } \\
\hline $8 / 21 / 03$ & Left bank & 4996502 & $11 \mathrm{TO} 670257$ & 4996665 & $11 \mathrm{TO} 668865$ \\
\hline $8 / 21 / 03$ & Right bank & 4996502 & $11 \mathrm{TO} 670257$ & 4996665 & $11 \mathrm{TO} 668865$ \\
\hline \multicolumn{6}{|c|}{ W.F. Chamberlain Creek } \\
\hline $8 / 21 / 03$ & Entire stream & 5027529 & 11 TO641790 & 5027785 & $11 \mathrm{TO} 641514$ \\
\hline \multicolumn{6}{|c|}{ Chamberlain Creek } \\
\hline $8 / 22 / 03$ & Entire stream & 5026359 & $11 \mathrm{TO} 642252$ & 5026109 & $11 \mathrm{TO} 642036$ \\
\hline \multicolumn{6}{|c|}{ Rush Creek } \\
\hline $8 / 22 / 03$ & Entire stream & 4996786 & 11 TO668323 & 4996466 & 11 TO668198 \\
\hline \multicolumn{6}{|c|}{ S. Fork Salmon River } \\
\hline $8 / 25 / 03$ & Right bank & 4943869 & $11 \mathrm{TO} 603567$ & 4943376 & 11 TO603594 \\
\hline $8 / 25 / 03$ & Left bank & 4943869 & $11 \mathrm{TO} 603567$ & 4943376 & 11 TO603594 \\
\hline $8 / 26 / 03$ & Right bank & 4940570 & 11 TO604714 & 4940319 & $11 \mathrm{TO} 604772$ \\
\hline $8 / 26 / 03$ & Left bank & 4940570 & 11 TO604714 & 4940319 & $11 \mathrm{TO} 604772$ \\
\hline \multicolumn{6}{|c|}{ Secesh River } \\
\hline $8 / 28 / 03$ & Left bank & 5006282 & 11TO593137 & 5007224 & 11 TO593492 \\
\hline $8 / 28 / 03$ & Right bank & 5006282 & $11 \mathrm{TO593137}$ & 5007224 & 11 TO593492 \\
\hline $8 / 29 / 03$ & Right bank & 5007224 & 11 TO593492 & 5008085 & $11 \mathrm{TO} 593646$ \\
\hline $8 / 29 / 03$ & Left bank & 5007224 & 11 TO593492 & 5008085 & 11 TO593646 \\
\hline \multicolumn{6}{|c|}{ Lake Creek } \\
\hline $8 / 30 / 03$ & Left bank & 5012376 & $11 \mathrm{TO586105}$ & 5013008 & 11TO60585 \\
\hline $8 / 30 / 03$ & Right bank & 5012376 & $11 \mathrm{TO} 586105$ & 5013048 & $11 \mathrm{TO} 58577$ \\
\hline
\end{tabular}


Appendix Table 3. Summary of observed total mortality for PIT-tagged wild Chinook salmon parr collected from Idaho streams during July and August 2003. Number rejected includes; fish too small to tag, precocious males, injured fish, fish collected for genetic evaluation, previously tagged fish, and in some cases extra collected fish. The portion of rejects that are precocious males are in parentheses.

\begin{tabular}{|c|c|c|c|c|c|c|c|c|}
\hline & \multirow[b]{2}{*}{$\begin{array}{l}\text { Number } \\
\text { collected }\end{array}$} & \multirow[b]{2}{*}{$\begin{array}{c}\text { Number } \\
\text { tagged }\end{array}$} & \multirow[b]{2}{*}{$\begin{array}{l}\text { Number } \\
\text { rejected }\end{array}$} & \multirow[b]{2}{*}{$\begin{array}{c}\text { Percent } \\
\text { rejected }(\%)\end{array}$} & \multicolumn{4}{|c|}{ Observed mortality } \\
\hline & & & & & $\begin{array}{c}\text { Collection \& } \\
\text { handling }\end{array}$ & $\begin{array}{c}\text { Post tagging } \\
\text { delayed }\end{array}$ & Number & Percent (\%) \\
\hline Bear Valley Creek & & & & 24.4 & 5 & 2 & 7 & 0.4 \\
\hline Elk Creek & 1,819 & 1,522 & $272(0)$ & 15.0 & 25 & 2 & 27 & 1.5 \\
\hline Marsh Creek & 1,812 & 1,535 & $268(0)$ & 14.8 & 9 & 0 & 9 & 0.6 \\
\hline Sulphur Creek & 1,319 & 1,053 & $264(6)$ & 20.0 & 2 & 5 & 7 & 0.5 \\
\hline Cape Horn Creek & 2,319 & 671 & $1,628(15)$ & 70.2 & 20 & 0 & 20 & 0.9 \\
\hline Valley Creek & 3,152 & 2,504 & $630(15)$ & 20.0 & 18 & 6 & 24 & 0.8 \\
\hline Loon Creek & 952 & 861 & $87(0)$ & 9.1 & 4 & 1 & 5 & 0.5 \\
\hline Camas Creek & 1,201 & 1,007 & $178(1)$ & 14.8 & 16 & 2 & 18 & 1.5 \\
\hline Herd Creek & 1,047 & 968 & $51(0)$ & 4.9 & 28 & 0 & 28 & 2.7 \\
\hline Big Creek (upper) & 1,933 & 1,504 & $403(13)$ & 20.8 & 26 & 0 & 26 & 1.3 \\
\hline Big Creek (lower) & 943 & 899 & $0(0)$ & 0.0 & 44 & 0 & 44 & 4.7 \\
\hline Rush Creek & 59 & 52 & $7(0)$ & 11.9 & 7 & 0 & 7 & 11.9 \\
\hline W.F. Chamberlain & 997 & 756 & $241(5)$ & 24.2 & 0 & 3 & 3 & 0.3 \\
\hline Chamberlain Creek & 316 & 243 & $66(2)$ & 20.9 & 7 & 0 & 7 & 2.2 \\
\hline S.F. Salmon River & 2,608 & 1,490 & $1,106(3)$ & 42.4 & 12 & 0 & 12 & 0.5 \\
\hline Secesh River & 1,708 & 1,146 & $544(3)$ & 31.9 & 18 & 4 & 22 & 1.3 \\
\hline Lake Creek & 1,029 & 664 & $364(2)$ & 35.4 & 1 & 0 & 1 & 0.1 \\
\hline Totals or averages & 25,209 & 18,371 & 6,596 & 26.2 & 242 & 25 & 267 & 1.1 \\
\hline
\end{tabular}


Appendix Table 4. Detections during 2004 of PIT-tagged smolts by date at three Snake River dams and three Columbia River dams for 1,494 wild Chinook salmon from Bear Valley Creek released 22-24 July 2003. Release sites were 629-634 km above Lower Granite Dam.

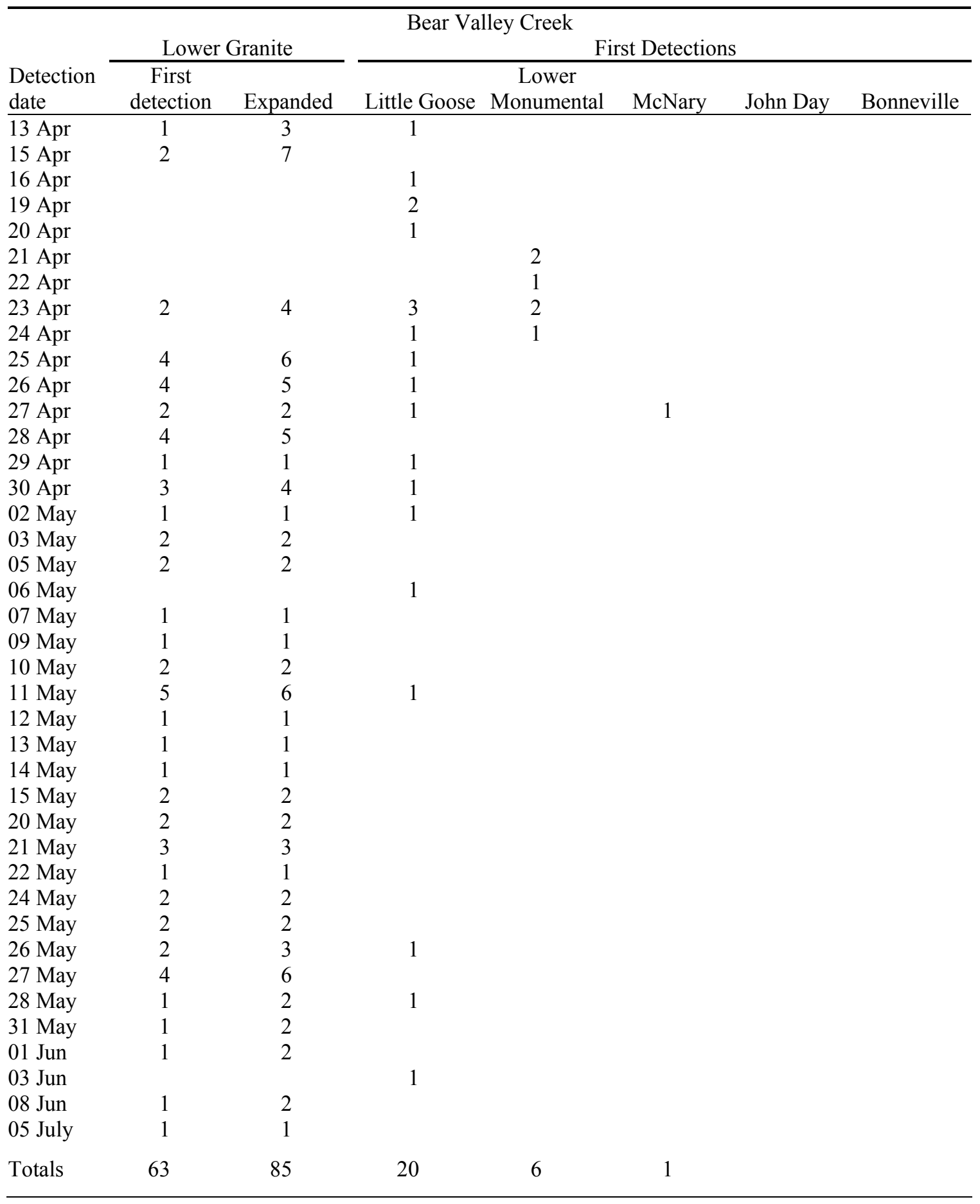


Appendix Table 5. Detections during 2004 of PIT-tagged smolts by date at three Snake River dams and three Columbia River dams for 1,520 wild Chinook salmon from Elk Creek released 25-29 July 2003. Release sites were 634-635 km above Lower Granite Dam.

\begin{tabular}{|c|c|c|c|c|c|c|c|}
\hline \multirow[b]{3}{*}{$\begin{array}{l}\text { Detection } \\
\text { date }\end{array}$} & \multicolumn{6}{|c|}{ Elk Creek } & \multirow[b]{3}{*}{ Bonneville } \\
\hline & \multicolumn{2}{|c|}{ Lower Granite } & \multicolumn{4}{|c|}{ First Detections } & \\
\hline & $\begin{array}{c}\text { First } \\
\text { detection }\end{array}$ & Expanded & Little Goose & $\begin{array}{c}\text { Lower } \\
\text { Monumental }\end{array}$ & McNary & John Day & \\
\hline 13-Apr & & & 1 & & & & \\
\hline 14-Apr & 1 & 3 & & & & & \\
\hline 15-Apr & 1 & 4 & & & & & \\
\hline 16-Apr & 1 & 3 & 1 & & & & \\
\hline 18-Apr & 1 & 3 & & & & & \\
\hline 19-Apr & 1 & 4 & 1 & & & & \\
\hline 20-Apr & & & 1 & & & & \\
\hline 21-Apr & 1 & 2 & & & & & \\
\hline 22-Apr & & & & 2 & & & \\
\hline 23-Apr & 3 & 6 & 2 & 1 & & & \\
\hline 24-Apr & 2 & 4 & 2 & & & & \\
\hline 25-Apr & & & 2 & & & & \\
\hline 26-Apr & 3 & 4 & & & & & \\
\hline 27-Apr & 2 & 2 & & 1 & & & \\
\hline 28-Apr & 1 & 1 & 1 & & & & \\
\hline 29-Apr & 5 & 6 & & & 1 & & \\
\hline 30-Apr & 4 & 5 & & & & & \\
\hline 02-May & 1 & 1 & 1 & & & & \\
\hline 03-May & 2 & 2 & & & & & \\
\hline 04-May & 2 & 2 & & & & & \\
\hline 05-May & & & & & & 1 & \\
\hline 06-May & 2 & 3 & & & & & \\
\hline 07-May & 1 & 1 & & & 1 & & \\
\hline 08-Мay & 3 & 4 & & & & & \\
\hline 09-May & 4 & 5 & & & & & \\
\hline 10-May & 2 & 2 & & & & & \\
\hline 11-May & 2 & 2 & & & & & \\
\hline 12-Мay & 1 & 1 & 1 & & & & \\
\hline 13-May & 2 & 2 & & & & & \\
\hline 14-May & 1 & 1 & & & & & \\
\hline 15-May & 1 & 1 & & & & & \\
\hline 16-Мay & 1 & 1 & & & & & \\
\hline 17-May & 1 & 1 & & & & & \\
\hline
\end{tabular}


Appendix Table 5. Continued.

\begin{tabular}{|c|c|c|c|c|c|c|c|}
\hline \multirow[b]{3}{*}{$\begin{array}{l}\text { Detection } \\
\text { date }\end{array}$} & \multicolumn{6}{|c|}{ Elk Creek (continued) } & \\
\hline & \multicolumn{2}{|c|}{ Lower Granite } & \multicolumn{5}{|c|}{ First Detections } \\
\hline & $\begin{array}{c}\text { First } \\
\text { detection }\end{array}$ & Expanded & $\begin{array}{l}\text { Little } \\
\text { Goose }\end{array}$ & $\begin{array}{c}\text { Lower } \\
\text { Monumental }\end{array}$ & McNary & John Day & Bonneville \\
\hline 18-May & 4 & 5 & & & & & \\
\hline 19-May & 1 & 1 & & & & & \\
\hline 20-May & & & 1 & & & & \\
\hline 21-May & 3 & 3 & & 1 & & & \\
\hline 22-May & 1 & 1 & & & & & \\
\hline 23-May & 5 & 6 & & & & & \\
\hline 26-May & 4 & 5 & & & & & \\
\hline 29-May & 1 & 2 & & & & & \\
\hline 31-May & 1 & 2 & & & & & \\
\hline 05-Jun & 1 & 2 & 1 & & & & \\
\hline 06-Jun & & & 3 & & & & \\
\hline 09-Jun & 1 & 2 & & & & & \\
\hline 12-Jun & 2 & 2 & 1 & & & & \\
\hline 13-Jun & 1 & 1 & & & & & \\
\hline 14-Jun & 1 & 1 & & & & & \\
\hline 17-Jun & 1 & 1 & & & & & \\
\hline 26-Jun & 1 & 1 & & & & & \\
\hline 12-Jul & 1 & 1 & & & & & \\
\hline Totals & 83 & 116 & 19 & 5 & 2 & 1 & \\
\hline
\end{tabular}


Appendix Table 6. Detections during 2004 of PIT-tagged smolts by date at three Snake River dams and three Columbia River dams for 1,535 wild Chinook salmon from Marsh Creek released 30 July-01 August 2003. Release sites were 630-632 km above Lower Granite Dam.

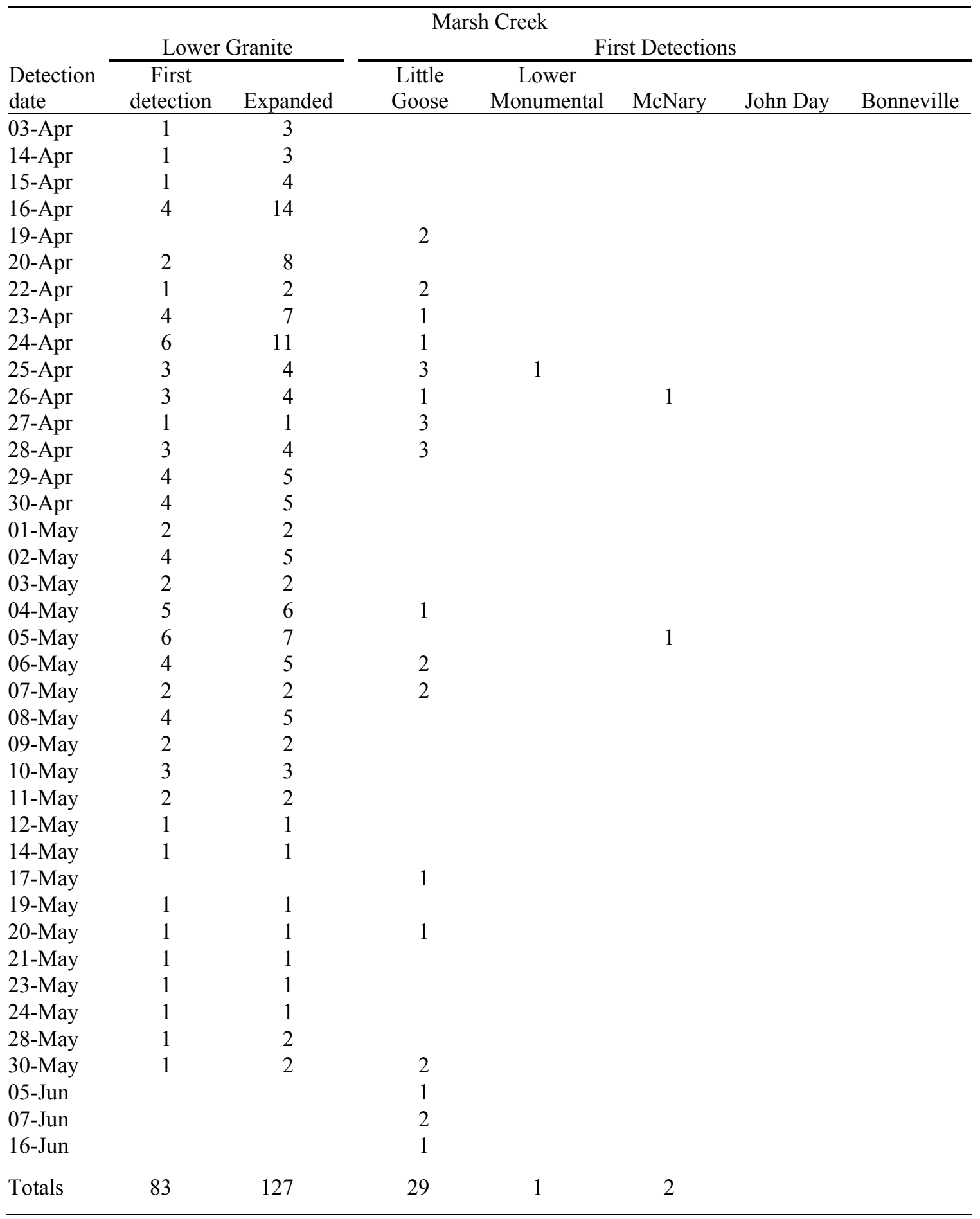


Appendix Table 7. Detections during 2004 of PIT-tagged smolts by date at three Snake River dams and three Columbia River dams for 671 wild Chinook salmon from Cape Horn Creek released 01-02 August 2003. Release sites were 629-636 km above Lower Granite Dam.

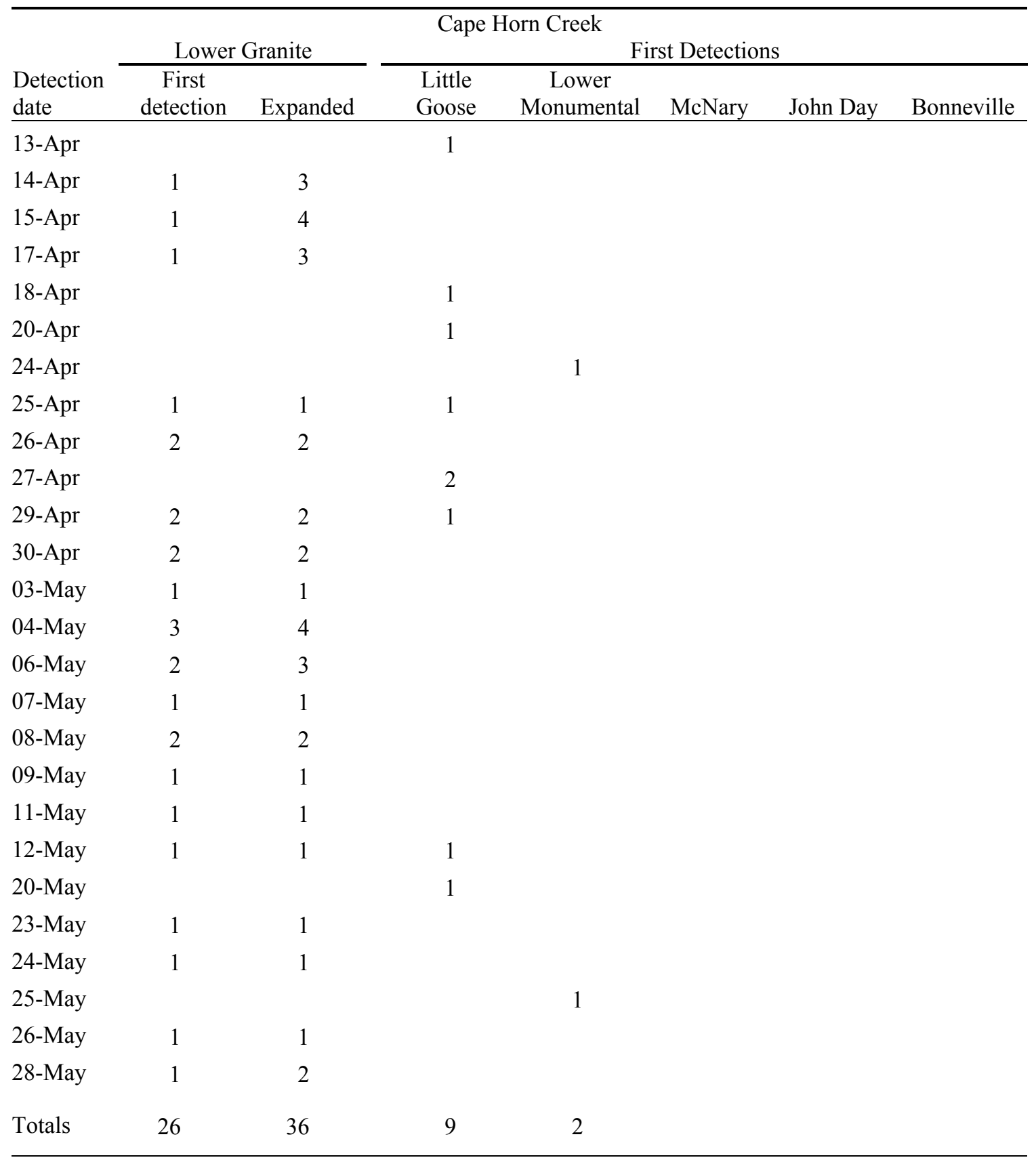


Appendix Table 8. Detections during 2004 of PIT-tagged smolts by date at three Snake River dams and three Columbia River dams for 1,048 wild Chinook salmon from Sulphur Creek released 31 July 2003. Fish were released $604 \mathrm{~km}$ above Lower Granite Dam.

\begin{tabular}{|c|c|c|c|c|c|c|c|}
\hline & & & & ur Creek & & & \\
\hline & Lower & Iranite & & & st Detectio & & \\
\hline $\begin{array}{l}\text { Detection } \\
\text { date }\end{array}$ & $\begin{array}{c}\text { First } \\
\text { detection }\end{array}$ & Expanded & $\begin{array}{c}\text { Little } \\
\text { Goose }\end{array}$ & $\begin{array}{c}\text { Lower } \\
\text { Monumental }\end{array}$ & McNary & John Day & Bonneville \\
\hline 02-Apr & 1 & 3 & & & & & \\
\hline 10-Apr & 1 & 3 & & & & & \\
\hline 15-Apr & 1 & 4 & & & & & \\
\hline 17-Apr & & & 1 & & & & \\
\hline 16-Apr & 1 & 3 & & & & & \\
\hline 19-Apr & 1 & 4 & & & & & \\
\hline 24-Apr & & & 1 & & & & \\
\hline 25-Apr & 3 & 4 & 1 & & & & \\
\hline 26-Apr & 2 & 2 & & & & & \\
\hline 27-Apr & & & 2 & & & & \\
\hline 28-Apr & 1 & 1 & 2 & & & & \\
\hline 29-Apr & 1 & 1 & & 1 & 1 & & \\
\hline 01-May & 1 & 1 & & & & & \\
\hline 03-May & 1 & 1 & & & 1 & & \\
\hline 04-May & 1 & 1 & & & & & \\
\hline 06-May & 1 & 1 & & & & & \\
\hline 07-May & 1 & 1 & & & & & \\
\hline 08-May & 3 & 4 & 2 & & & & \\
\hline 10-May & 1 & 1 & & & & & \\
\hline 11-May & 2 & 2 & & & & & \\
\hline 21-May & 1 & 1 & & & & & \\
\hline 22-May & 1 & 1 & & & & & \\
\hline 24-May & 1 & 1 & & & & & \\
\hline Totals & 26 & 40 & 9 & 1 & 2 & & \\
\hline
\end{tabular}


Appendix Table 9. Detections during 2004 of PIT-tagged smolts by date at three Snake River dams and three Columbia River dams for 2,498 wild Chinook salmon from Valley Creek released 04-06 August 2003. Release sites were 743-756 km above Lower Granite Dam.

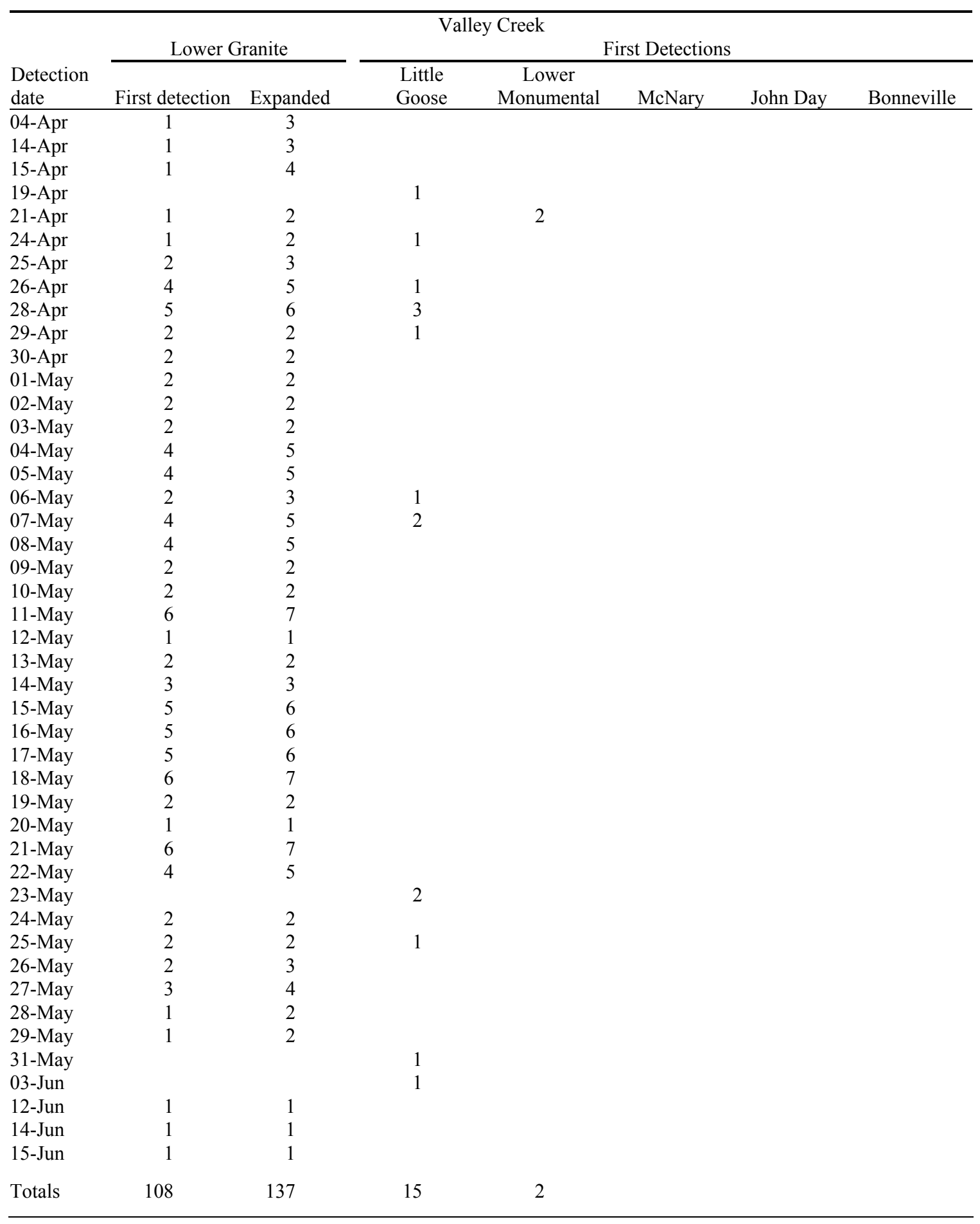


Appendix Table 10. Detections during 2004 of PIT-tagged smolts by date at three Snake River dams and three Columbia River dams for 860 wild Chinook salmon from Loon Creek released 08-09 August 2003. Release sites were 555-559 km above Lower Granite Dam.

\begin{tabular}{|c|c|c|c|c|c|c|c|}
\hline \multirow[b]{3}{*}{$\begin{array}{l}\text { Detection } \\
\text { date }\end{array}$} & \multicolumn{6}{|c|}{ Loon Creek } & \multirow[b]{3}{*}{ Bonneville } \\
\hline & \multicolumn{2}{|c|}{ Lower Granite } & \multicolumn{4}{|c|}{ First Detections } & \\
\hline & $\begin{array}{c}\text { First } \\
\text { detection }\end{array}$ & Expanded & $\begin{array}{c}\text { Little } \\
\text { Goose }\end{array}$ & $\begin{array}{c}\text { Lower } \\
\text { Monumental }\end{array}$ & McNary & John Day & \\
\hline $15-\mathrm{Apr}$ & 1 & 4 & & & & & \\
\hline 16-Apr & & & 1 & & & & \\
\hline 17-Apr & 1 & 3 & & & & & \\
\hline 19-Apr & 1 & 4 & & & & & \\
\hline 23-Apr & 1 & 2 & & & & & \\
\hline 24-Apr & 2 & 4 & & & & & \\
\hline 25-Apr & 3 & 4 & & & & & \\
\hline 26-Apr & 2 & 2 & & & & & \\
\hline 27-Apr & 2 & 2 & & & & & \\
\hline 28-Apr & 5 & 6 & 4 & & & & \\
\hline 29-Apr & 2 & 2 & 1 & & & & \\
\hline 30-Apr & 2 & 2 & & & & & \\
\hline 01-May & 3 & 4 & & & & & \\
\hline 03-May & 5 & 6 & & & & & \\
\hline 04-May & 6 & 7 & 1 & & 1 & & \\
\hline 05-May & 12 & 14 & 2 & & & & \\
\hline 06-May & 1 & 1 & 1 & & & & \\
\hline 07-May & 4 & 5 & & & & & \\
\hline 08-May & 7 & 8 & & & & & \\
\hline 09-May & 4 & 5 & & & & & \\
\hline 10-May & 11 & 12 & 1 & & & & \\
\hline 11-May & 2 & 2 & & & & & \\
\hline 12-May & & & 2 & & & & \\
\hline 13-May & 1 & 1 & & & & & \\
\hline 14-May & 1 & 1 & 2 & & & & \\
\hline 15-May & 2 & 2 & & & & & \\
\hline 16-May & & & 2 & & & & \\
\hline 17-May & 1 & 1 & & & & & \\
\hline 18-May & 3 & 3 & & & & & \\
\hline 19-May & 1 & 1 & & & & & \\
\hline 20-May & 2 & 2 & & & & 1 & \\
\hline 22-May & 1 & 1 & & & & & \\
\hline 24-May & 1 & 1 & & & & & \\
\hline 26-May & 1 & 1 & & & & & \\
\hline 01-Jun & & & 1 & & & & \\
\hline 07-Jun & & & 1 & & & & \\
\hline Totals & 91 & 113 & 19 & & 1 & 1 & \\
\hline
\end{tabular}


Appendix Table 11. Detections during 2004 of PIT-tagged smolts by date at three Snake River dams and three Columbia River dams for 1,005 wild Chinook salmon from Camas Creek released 08-09 August 2003. Release sites were 526-528 km above Lower Granite Dam.

\begin{tabular}{|c|c|c|c|c|c|c|c|}
\hline \multirow[b]{3}{*}{$\begin{array}{l}\text { Detection } \\
\text { date }\end{array}$} & \multicolumn{6}{|c|}{ Camas Creek } & \multirow[b]{3}{*}{ Bonneville } \\
\hline & \multicolumn{2}{|c|}{ Lower Granite } & \multicolumn{4}{|c|}{ First Detections } & \\
\hline & $\begin{array}{c}\text { First } \\
\text { detection }\end{array}$ & Expanded & $\begin{array}{l}\text { Little } \\
\text { Goose }\end{array}$ & $\begin{array}{c}\text { Lower } \\
\text { Monumental }\end{array}$ & McNary & John Day & \\
\hline 16-Apr & 2 & 7 & & & & & \\
\hline 17-Apr & 1 & 3 & 1 & & & & \\
\hline 19-Apr & 1 & 4 & & & & & \\
\hline 20-Apr & 1 & 4 & & & & & \\
\hline 21-Apr & & & 1 & & & & \\
\hline 22-Apr & & & & 1 & & & \\
\hline 23-Apr & 1 & 2 & 1 & & & & \\
\hline 24-Apr & & & 3 & & & & \\
\hline 25-Apr & & & 2 & & & & \\
\hline 26-Apr & 5 & 6 & 2 & & & & \\
\hline 27-Apr & 2 & 2 & & & & & \\
\hline 28-Apr & 2 & 2 & 2 & & & & \\
\hline 29-Apr & 2 & 2 & & & & & \\
\hline 30-Apr & 2 & 2 & & & & & \\
\hline 01-May & 1 & 1 & 1 & & & & \\
\hline 02-May & 1 & 1 & & & & & \\
\hline 03-May & 1 & 1 & 1 & & & & \\
\hline 04-May & 6 & 7 & & & & & \\
\hline 05-May & 1 & 1 & & & & & \\
\hline 07-May & 2 & 2 & 1 & & 1 & & \\
\hline 08-May & 1 & 1 & & & 1 & & \\
\hline 09-May & 2 & 2 & & & & & \\
\hline 10-May & 5 & 6 & 2 & & & & \\
\hline 11-May & 5 & 6 & & & & & \\
\hline 12-May & 1 & 1 & & & & & \\
\hline 13-May & 1 & 1 & & & & & \\
\hline 14-May & 2 & 2 & & & & & \\
\hline 15-May & & & 1 & & & & \\
\hline 16-May & 1 & 1 & & & & & \\
\hline
\end{tabular}


Appendix Table 11. Continued.

\begin{tabular}{|c|c|c|c|c|c|c|c|}
\hline \multirow[b]{3}{*}{$\begin{array}{l}\text { Detection } \\
\text { date }\end{array}$} & \multicolumn{6}{|c|}{ Camas Creek (continued) } & \multirow[b]{3}{*}{ Bonneville } \\
\hline & \multicolumn{2}{|c|}{ Lower Granite } & \multicolumn{4}{|c|}{ First Detections } & \\
\hline & $\begin{array}{c}\text { First } \\
\text { detection }\end{array}$ & Expanded & $\begin{array}{l}\text { Little } \\
\text { Goose }\end{array}$ & $\begin{array}{c}\text { Lower } \\
\text { Monumental }\end{array}$ & McNary & John Day & \\
\hline 17-May & 2 & 2 & & & & & \\
\hline 18-May & 1 & 1 & & & & & \\
\hline 19-May & 3 & 3 & & & & & \\
\hline 20-May & 1 & 1 & 2 & & & & \\
\hline 21-May & 2 & 2 & 1 & & & & \\
\hline 22-May & 2 & 2 & & & & & \\
\hline 23-May & 2 & 2 & & & & & \\
\hline 24-May & 5 & 6 & & & & & \\
\hline 25-May & 2 & 2 & & & & & \\
\hline 26-May & 1 & 1 & & & & & \\
\hline 27-May & 1 & 1 & & & & & \\
\hline 28-May & 1 & 2 & & & & & \\
\hline 04-Jun & 1 & 2 & & & & & \\
\hline 05-Jun & & & 1 & & & & \\
\hline 07-Jun & & & & 1 & & & \\
\hline 09-Jun & & & 1 & & & & \\
\hline 13-Jun & & & & 1 & & & \\
\hline Totals & 73 & 94 & 23 & 3 & 2 & & \\
\hline
\end{tabular}


Appendix Table 12. Detections during 2004 of PIT-tagged smolts by date at three Snake River dams and three Columbia River dams for 968 wild Chinook salmon from Herd Creek released 11-12 August 2003. Fish were released $699 \mathrm{~km}$ above Lower Granite Dam.

\begin{tabular}{|c|c|c|c|c|c|c|c|}
\hline & & & & Creek & & & \\
\hline & Lower & Granite & & & st Detectio & & \\
\hline $\begin{array}{l}\text { Detection } \\
\text { date }\end{array}$ & $\begin{array}{c}\text { First } \\
\text { detection }\end{array}$ & Expanded & $\begin{array}{c}\text { Little } \\
\text { Goose }\end{array}$ & $\begin{array}{c}\text { Lower } \\
\text { Monumental }\end{array}$ & McNary & John Day & Bonneville \\
\hline 12-Apr & 2 & 6 & & & & & \\
\hline 14-Apr & 1 & 3 & & & & & \\
\hline 16-Apr & 1 & 3 & & & & & \\
\hline 18-Apr & 1 & 3 & 2 & & & & \\
\hline 19-Apr & & & 1 & & & & \\
\hline 20-Apr & & & 3 & 1 & & & \\
\hline 21-Apr & & & 1 & & & & \\
\hline 22-Apr & 2 & 4 & & & & & \\
\hline 23-Apr & 4 & 7 & 1 & & & & \\
\hline 24-Apr & & & 2 & & & & \\
\hline 25-Apr & 1 & 1 & 4 & & & & \\
\hline 26-Apr & 7 & 8 & 2 & & & & \\
\hline 27-Apr & 3 & 4 & 2 & & & & \\
\hline 28-Apr & 6 & 7 & 1 & & & & \\
\hline 29-Apr & 5 & 6 & 1 & & & & \\
\hline 30-Apr & 5 & 6 & & & & & \\
\hline 01-May & 3 & 4 & & & & & \\
\hline 02-May & 2 & 2 & & & & & \\
\hline 03-May & 2 & 2 & & & 2 & & \\
\hline 04-May & 5 & 6 & & & & & \\
\hline 05-May & 3 & 4 & 1 & & & & \\
\hline 06-May & 3 & 4 & & & & & \\
\hline 07-May & 5 & 6 & 2 & & & & \\
\hline 08-May & 2 & 2 & 2 & & 1 & & \\
\hline 09-May & 3 & 3 & 1 & & & & \\
\hline 10-May & 6 & 7 & & & & & \\
\hline 11-May & 2 & 2 & & & & & \\
\hline 13-May & 1 & 1 & & & & & \\
\hline 14-May & 1 & 1 & & & & & \\
\hline 15-May & & & & & & & 1 \\
\hline 17-May & & & & 1 & & & \\
\hline 18-May & 1 & 1 & & & & & \\
\hline 20-May & 1 & 1 & 1 & & & & \\
\hline 21-May & 1 & 1 & & & & & \\
\hline 22-May & 1 & 1 & 1 & & & & \\
\hline 21-Jun & 1 & 1 & & & & & \\
\hline Totals & 81 & 107 & 28 & 2 & 3 & & 1 \\
\hline
\end{tabular}


Appendix Table 13. Detections during 2004 of PIT-tagged smolts by date at three Snake River dams and three Columbia River dams for 1,504 wild Chinook salmon from Big Creek (upper) released 15-16 August 2003. Release sites were 530-533 km above Lower Granite Dam.

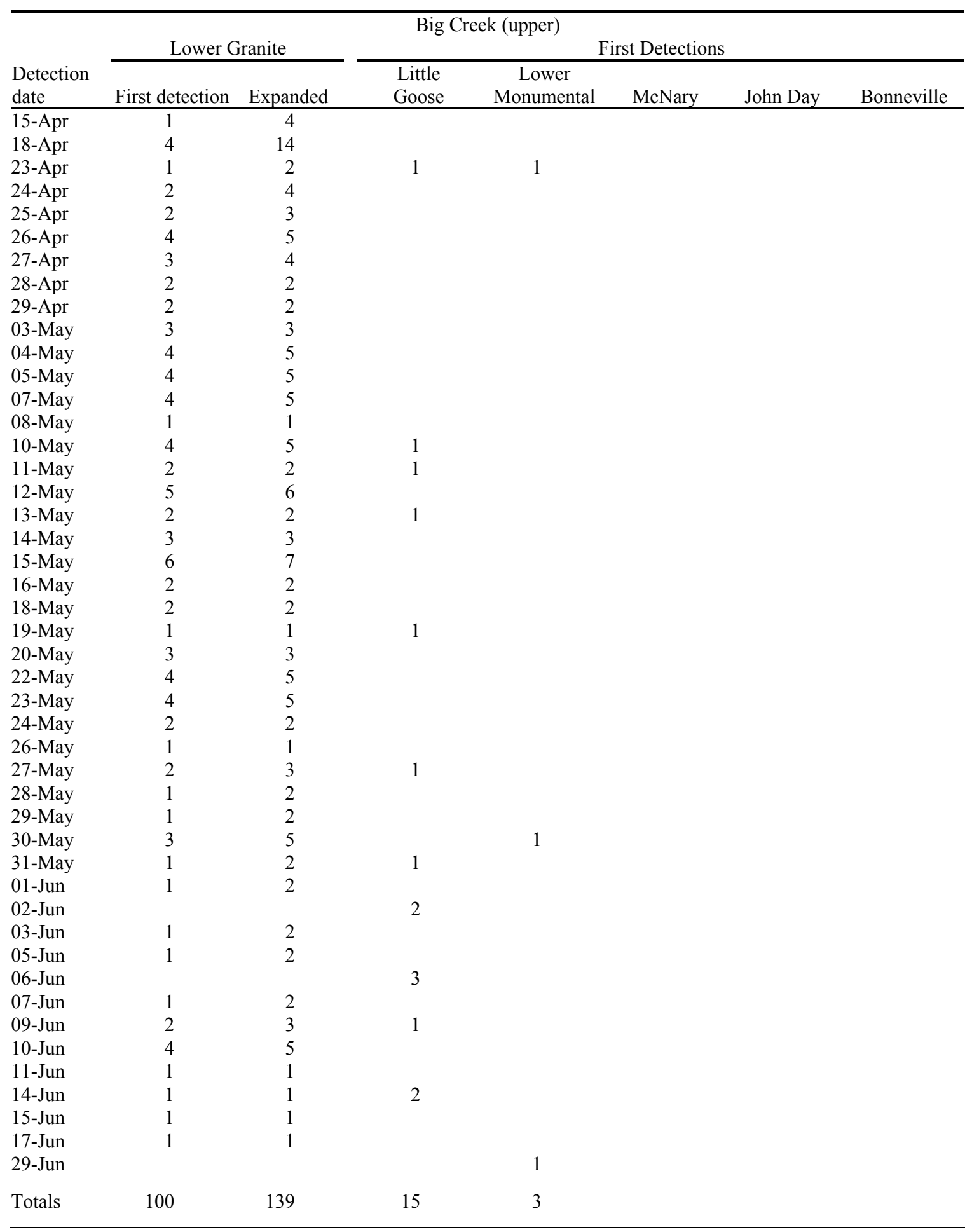


Appendix Table 14. Detections during 2004 of PIT-tagged smolts by date at three Snake River dams and three Columbia River dams for 951 wild Chinook salmon from Big (lower)/Rush Creeks released 21-22 August 2003. Release sites were 486-489 km above Lower Granite Dam.

\begin{tabular}{|c|c|c|c|c|c|c|c|}
\hline & & & ig Creek & ver)/Rush Cre & & & \\
\hline & Lower & Iranite & & & st Detectio & & \\
\hline $\begin{array}{l}\text { Detection } \\
\text { date }\end{array}$ & $\begin{array}{c}\text { First } \\
\text { detection }\end{array}$ & Expanded & $\begin{array}{l}\text { Little } \\
\text { Goose }\end{array}$ & $\begin{array}{c}\text { Lower } \\
\text { Monumental }\end{array}$ & McNary & John Day & Bonneville \\
\hline 06-Apr & 1 & 4 & & & & & \\
\hline 07-Apr & 1 & 2 & & & & & \\
\hline 14-Apr & 1 & 3 & & & & & \\
\hline 15-Apr & 5 & 18 & & & & & \\
\hline 16-Apr & 5 & 17 & & & & & \\
\hline 17-Apr & 1 & 3 & & & & & \\
\hline 18-Apr & 2 & 7 & 1 & & & & \\
\hline 19-Apr & 2 & 8 & 5 & & & & \\
\hline 20-Apr & 1 & 4 & 2 & & & & \\
\hline 21-Apr & 4 & 10 & 1 & 1 & & & \\
\hline 22-Apr & 2 & 4 & & & & & \\
\hline 23-Apr & 4 & 7 & 3 & 2 & & & \\
\hline 24-Apr & 2 & 4 & 5 & 1 & 1 & & \\
\hline 25-Apr & 9 & 13 & 5 & 1 & & & \\
\hline 26-Apr & 7 & 8 & 1 & & & & \\
\hline 27-Apr & 2 & 2 & 1 & & & & \\
\hline 28-Apr & 9 & 11 & 1 & 1 & 2 & & \\
\hline 29-Apr & 3 & 4 & 1 & & & & \\
\hline 30-Apr & 8 & 10 & & & 2 & & \\
\hline 01-May & 3 & 4 & & & 3 & & \\
\hline 02-May & 1 & 1 & 2 & & 1 & & \\
\hline 03-May & 2 & 2 & & & 1 & & \\
\hline 04-May & 7 & 8 & & & 1 & & \\
\hline 05-May & 3 & 4 & & & & & \\
\hline 06-May & 1 & 1 & 1 & & & & \\
\hline 07-May & 4 & 5 & & & & & \\
\hline 08-May & 1 & 1 & & & & & \\
\hline 09-May & 2 & 2 & & & & & \\
\hline 10-May & 1 & 1 & & & & & \\
\hline 11-May & & & 1 & & & & \\
\hline 15-May & 1 & 1 & & & & & \\
\hline 28-May & & & & 1 & & & \\
\hline Totals & 95 & 169 & 30 & 7 & 11 & & \\
\hline
\end{tabular}


Appendix Table 15. Detections during 2004 of PIT-tagged smolts by date at three Snake River dams and three Columbia River dams for 996 wild Chinook salmon from West Fork Chamberlain Creek released 21-22 Aug. 2003. Release sites were 437-438 km above Lower Granite Dam.

\begin{tabular}{|c|c|c|c|c|c|c|c|}
\hline \multirow[b]{3}{*}{$\begin{array}{l}\text { Detection } \\
\text { date }\end{array}$} & \multicolumn{6}{|c|}{ West Fork Chamberlain Creek ${ }^{\mathrm{a}}$} & \multirow[b]{3}{*}{ Bonneville } \\
\hline & \multicolumn{2}{|c|}{ Lower Granite } & \multicolumn{4}{|c|}{ First Detections } & \\
\hline & $\begin{array}{c}\text { First } \\
\text { detection }\end{array}$ & Expanded & $\begin{array}{c}\text { Little } \\
\text { Goose }\end{array}$ & $\begin{array}{c}\text { Lower } \\
\text { Monumental }\end{array}$ & McNary & John Day & \\
\hline 07-Apr & 1 & 2 & & & & & \\
\hline 09-Apr & 1 & 3 & & & & & \\
\hline 11-Apr & 1 & 4 & & & & & \\
\hline 13-Apr & 1 & 3 & & & & & \\
\hline 14-Apr & 1 & 3 & & & & & \\
\hline 15-Apr & 2 & 7 & & & & & \\
\hline 17-Apr & 2 & 7 & & & & & \\
\hline 18-Apr & 1 & 3 & & & & & \\
\hline 19-Apr & & & 1 & & & & \\
\hline 20-Apr & & & 1 & 1 & & & \\
\hline 21-Apr & & & 1 & & & & \\
\hline 22-Apr & 1 & 2 & & & & & \\
\hline 23-Apr & 3 & 6 & & & & & \\
\hline 24-Apr & 3 & 5 & 4 & & & & \\
\hline 25-Apr & 2 & 3 & & & & & \\
\hline 27-Apr & 2 & 2 & 1 & & & & \\
\hline 29-Apr & 4 & 5 & & & & & \\
\hline 30-Apr & 1 & 1 & & & & & \\
\hline 01-May & 1 & 1 & & & & & \\
\hline 02-May & 1 & 1 & & & & & \\
\hline 03-May & 2 & 2 & & & & & \\
\hline 04-May & 3 & 4 & & & & & \\
\hline 05-May & 3 & 4 & & & & & \\
\hline 06-May & 2 & 3 & & & & & \\
\hline 09-May & 1 & 1 & & & & & \\
\hline 10-May & 2 & 2 & & & & & \\
\hline 15-May & 1 & 1 & & & & & \\
\hline 16-May & 1 & 1 & & & & & \\
\hline 17-May & 1 & 1 & 1 & & & & \\
\hline 22-May & 1 & 1 & & & & & \\
\hline 23-May & 1 & 1 & & & & & 1 \\
\hline 25-May & 1 & 1 & & & & & \\
\hline 26-May & & & 1 & & & & \\
\hline 18-Jun & & & 1 & & & & \\
\hline 23-Jun & 1 & 1 & & & & & \\
\hline Totals & 48 & 81 & 11 & 1 & & & 1 \\
\hline
\end{tabular}

a Includes fish from Chamberlain Creek 
Appendix Table 16. Detections during 2004 of PIT-tagged smolts by date at three Snake River dams and three Columbia River dams for 1,490 wild Chinook salmon from South Fork Salmon River released 25-26 Aug. 2003. Release sites were 467-473 km above Lower Granite Dam.

\begin{tabular}{|c|c|c|c|c|c|c|c|}
\hline \multirow[b]{3}{*}{$\begin{array}{l}\text { Detection } \\
\text { date }\end{array}$} & \multicolumn{6}{|c|}{ South Fork Salmon River } & \multirow[b]{3}{*}{ Bonneville } \\
\hline & \multicolumn{2}{|c|}{ Lower Granite } & \multicolumn{4}{|c|}{$\begin{array}{ll}\text { First Detections } \\
\end{array}$} & \\
\hline & $\begin{array}{c}\text { First } \\
\text { detection }\end{array}$ & Expanded & $\begin{array}{c}\text { Little } \\
\text { Goose }\end{array}$ & $\begin{array}{c}\text { Lower } \\
\text { Monumental }\end{array}$ & McNary & John Day & \\
\hline 08-Apr & 1 & 2 & & & & & \\
\hline 15-Apr & 1 & 4 & & & & & \\
\hline 16-Apr & 2 & 7 & & & & & \\
\hline 17-Apr & & & & 1 & & & \\
\hline 18-Apr & 1 & 3 & & & & & \\
\hline 20-Apr & & & 1 & & & & \\
\hline 21-Apr & & & & 1 & & & \\
\hline 22-Apr & & & & 1 & & & \\
\hline 24-Apr & 1 & 2 & 1 & 1 & & & \\
\hline 25-Apr & 1 & 1 & & & & & \\
\hline 26-Apr & 1 & 1 & 1 & & & & \\
\hline 27-Apr & 1 & 1 & & & & & \\
\hline 28-Apr & 1 & 1 & 1 & & 1 & & \\
\hline 01-May & 2 & 2 & & & & & \\
\hline 03-May & 1 & 1 & & & & & \\
\hline 04-May & 5 & 6 & & & & & \\
\hline 05-May & 2 & 2 & & & & & \\
\hline 06-May & 2 & 3 & & & & & \\
\hline 07-May & 4 & 5 & & & & & \\
\hline 08-May & 2 & 2 & & & & & \\
\hline 09-Мay & 1 & 1 & & & & & \\
\hline 10-May & 5 & 6 & 1 & & & & \\
\hline 11-May & 3 & 3 & & & & & \\
\hline 12-May & 1 & 1 & & & & & \\
\hline 14-May & 1 & 1 & & 1 & & & \\
\hline 15-May & & & 2 & & & & \\
\hline 16-May & 5 & 6 & & & & & \\
\hline 18-May & & & 4 & & & & \\
\hline
\end{tabular}


Appendix Table 16. Continued.

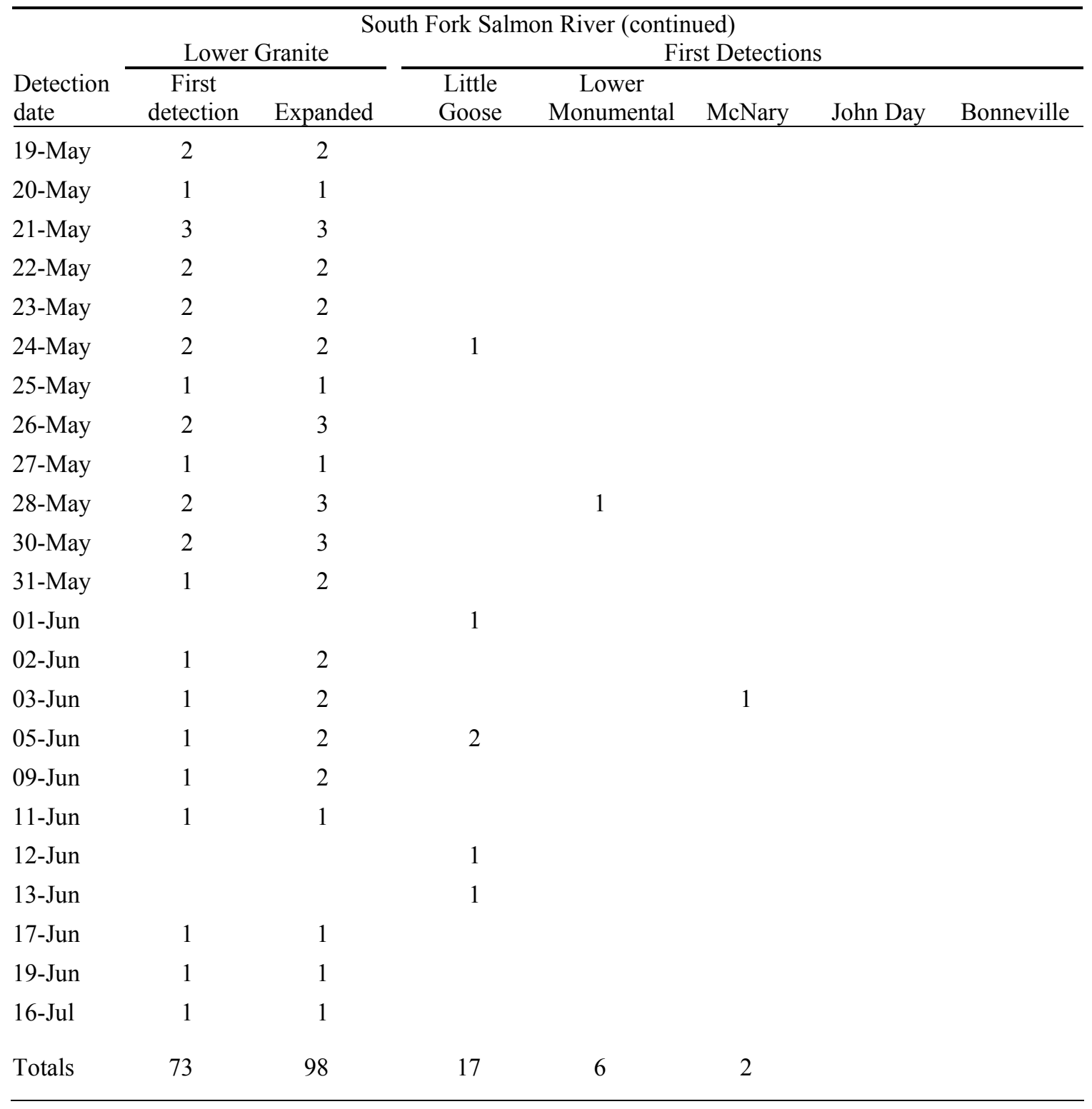


Appendix Table 17. Detections during 2004 of PIT-tagged smolts by date at three Snake River dams and three Columbia River dams for 1,142 wild Chinook salmon from Secesh River released 28-29 Aug. 2003. Release sites were 429-431 km above Lower Granite Dam.

\begin{tabular}{|c|c|c|c|c|c|c|c|}
\hline \multirow[b]{3}{*}{$\begin{array}{l}\text { Detection } \\
\text { date }\end{array}$} & \multicolumn{6}{|c|}{ Secesh River } & \multirow[b]{3}{*}{ Bonneville } \\
\hline & \multicolumn{2}{|c|}{ Lower Granite } & \multicolumn{4}{|c|}{ First Detections } & \\
\hline & $\begin{array}{c}\text { First } \\
\text { detection }\end{array}$ & Expanded & $\begin{array}{l}\text { Little } \\
\text { Goose }\end{array}$ & $\begin{array}{c}\text { Lower } \\
\text { Monumental }\end{array}$ & McNary & John Day & \\
\hline 01-Apr & 1 & 5 & & & & & \\
\hline 08-Apr & 1 & 2 & & & & & \\
\hline 15-Apr & 1 & 4 & & & & & \\
\hline 17-Apr & & & & 1 & & & \\
\hline 18-Apr & & & 3 & & & & \\
\hline 20-Apr & 1 & 4 & & & & & \\
\hline 21-Apr & & & 1 & & & & \\
\hline 22-Apr & 1 & 2 & & 1 & & & \\
\hline 23-Apr & & & & 1 & & & \\
\hline 24-Apr & 1 & 2 & 2 & 1 & & & \\
\hline 25-Apr & 1 & 1 & & & & & \\
\hline 26-Apr & 2 & 2 & & & & & \\
\hline 27-Apr & 2 & 2 & 1 & & & & \\
\hline 28-Apr & 1 & 1 & & & & & \\
\hline 29-Apr & 1 & 1 & & & & & \\
\hline 01-May & & & & & 1 & & \\
\hline 02-May & 2 & 2 & 1 & & & & \\
\hline 04-May & 1 & 1 & & & & & \\
\hline 05-May & 1 & 1 & & & & & \\
\hline 07-May & & & 1 & & & & \\
\hline 10-May & 1 & 1 & & & & & \\
\hline 14-May & 1 & 1 & & & & & \\
\hline 15-May & 1 & 1 & & & & & \\
\hline 16-May & 1 & 1 & & & & & \\
\hline 21-May & 2 & 2 & & & & & \\
\hline 22-May & 1 & 1 & & & & & \\
\hline 24-May & 1 & 1 & & & & & \\
\hline 28-May & 2 & 3 & & & & & \\
\hline 06-Jun & & & 1 & & & & \\
\hline 08-Jun & 1 & 2 & & & & & \\
\hline 10-Jun & 1 & 1 & & & & & \\
\hline 13-Jun & 1 & 1 & 1 & & & & \\
\hline Totals & 30 & 45 & 11 & 4 & 1 & & \\
\hline
\end{tabular}


Appendix Table 18. Detections during 2004 of PIT-tagged smolts by date at three Snake River dams and three Columbia River dams for 664 wild Chinook salmon from Lake Creek released 30 Aug. 2003. Release sites were 451-452 km above Lower Granite Dam.

\begin{tabular}{|c|c|c|c|c|c|c|c|}
\hline & & & & Creek & & & \\
\hline & Lower & Iranite & & & st Detectio & & \\
\hline $\begin{array}{l}\text { Detection } \\
\text { date }\end{array}$ & $\begin{array}{c}\text { First } \\
\text { detection }\end{array}$ & Expanded & $\begin{array}{c}\text { Little } \\
\text { Goose }\end{array}$ & $\begin{array}{c}\text { Lower } \\
\text { Monumental }\end{array}$ & McNary & John Day & Bonneville \\
\hline 09-Apr & 1 & 3 & & & & & \\
\hline 14-Apr & 1 & 3 & & & & & \\
\hline 15-Apr & 2 & 7 & & 1 & & & \\
\hline 16-Apr & 2 & 7 & & & & & \\
\hline 21-Apr & & & 1 & & & & \\
\hline 22-Apr & & & & 1 & & & \\
\hline 25-Apr & 2 & 3 & & & & & \\
\hline 26-Apr & 2 & 2 & & & & & \\
\hline 27-Apr & & & 1 & & & & \\
\hline 30-Apr & 1 & 1 & & & & & \\
\hline 04-May & 2 & 2 & & & & & \\
\hline 09-May & 1 & 1 & & & & & \\
\hline 10-May & 1 & 1 & & & & & \\
\hline 12-May & 1 & 1 & & & & & \\
\hline 14-May & 2 & 2 & & & & & \\
\hline 16-May & 1 & 1 & & & & & \\
\hline 19-May & 1 & 1 & & & & & \\
\hline 22-May & 1 & 1 & & & & & \\
\hline 23-May & 1 & 1 & & & & & \\
\hline 28-May & 2 & 3 & & & & & \\
\hline 04-Jun & & & 1 & & & & \\
\hline 05-Jun & & & 1 & & & & \\
\hline 06-Jun & & & 1 & & & & \\
\hline 11-Jun & 1 & 1 & & & & & \\
\hline 16-Jun & 1 & 1 & & & & & \\
\hline Totals & 26 & 42 & 5 & 2 & & & \\
\hline
\end{tabular}


Appendix Table 19. Daily and expanded detections of PIT-tagged wild spring/summer Chinook salmon smolts from Idaho and Oregon at Lower Granite Dam during 2004, with associated river flows ( $\mathrm{kcfs}$ ), spill ( $\mathrm{kcfs}$ ), and water temperatures $\left({ }^{\circ} \mathrm{C}\right)$ at the dam.

\begin{tabular}{|c|c|c|c|c|c|c|c|}
\hline \multirow[b]{2}{*}{ Date } & \multirow[b]{2}{*}{$\begin{array}{c}\text { Average } \\
\text { flow (kcfs) }\end{array}$} & \multirow[b]{2}{*}{$\begin{array}{c}\text { Average } \\
\text { spill (kcfs) }\end{array}$} & \multirow[b]{2}{*}{$\begin{array}{l}\text { Scroll-case } \\
\text { water } \\
\text { temperature }\end{array}$} & \multicolumn{2}{|c|}{ Idaho only } & \multicolumn{2}{|c|}{ Idaho and Oregon } \\
\hline & & & & $\begin{array}{l}\text { Numbers } \\
\text { detected }\end{array}$ & $\begin{array}{l}\text { Expanded } \\
\text { numbers } \\
\text { detected }\end{array}$ & $\begin{array}{l}\text { Numbers } \\
\text { detected }\end{array}$ & $\begin{array}{c}\text { Expanded } \\
\text { numbers } \\
\text { detected }\end{array}$ \\
\hline $01 \mathrm{Apr}$ & 50.4 & 0.0 & 8.9 & 1 & 5 & 1 & 5 \\
\hline $02 \mathrm{Apr}$ & 48.2 & 0.0 & 8.9 & 1 & 3 & 1 & 3 \\
\hline $03 \mathrm{Apr}$ & 39.3 & 4.6 & 8.9 & 1 & 3 & 1 & 3 \\
\hline $04 \mathrm{Apr}$ & 44.9 & 18.2 & 10.0 & 1 & 3 & 1 & 3 \\
\hline $06 \mathrm{Apr}$ & 44.8 & 19.3 & 10.0 & 1 & 4 & 1 & 4 \\
\hline $07 \mathrm{Apr}$ & 48.8 & 18.8 & 10.0 & 2 & 4 & 3 & 7 \\
\hline $08 \mathrm{Apr}$ & 52.8 & 15.3 & 10.0 & 2 & 4 & 2 & 5 \\
\hline $09 \mathrm{Apr}$ & 54.7 & 24.3 & 11.1 & 2 & 6 & 2 & 6 \\
\hline $10 \mathrm{Apr}$ & 49.5 & 18.7 & 11.7 & 1 & 3 & 1 & 3 \\
\hline $11 \mathrm{Apr}$ & 49.2 & 18.7 & 12.2 & 1 & 4 & 1 & 4 \\
\hline $12 \mathrm{Apr}$ & 46.8 & 18.5 & 12.2 & 2 & 6 & 2 & 6 \\
\hline $13 \mathrm{Apr}$ & 54.6 & 18.3 & 11.7 & 2 & 6 & 2 & 5 \\
\hline $14 \mathrm{Apr}$ & 64.2 & 18.4 & 11.7 & 8 & 24 & 9 & 31 \\
\hline $15 \mathrm{Apr}$ & 64.2 & 18.4 & 11.7 & 20 & 75 & 23 & 83 \\
\hline $16 \mathrm{Apr}$ & 56.6 & 18.3 & 11.7 & 18 & 61 & 21 & 72 \\
\hline $17 \mathrm{Apr}$ & 50.9 & 18.3 & 11.7 & 6 & 19 & 6 & 20 \\
\hline $18 \mathrm{Apr}$ & 49.6 & 18.3 & 11.1 & 9 & 30 & 11 & 38 \\
\hline $19 \mathrm{Apr}$ & 46.8 & 18.3 & 10.6 & 6 & 24 & 9 & 37 \\
\hline $20 \mathrm{Apr}$ & 45.2 & 18.4 & 10.6 & 5 & 20 & 6 & 24 \\
\hline $21 \mathrm{Apr}$ & 43.8 & 18.5 & 10.6 & 6 & 14 & 7 & 17 \\
\hline $22 \mathrm{Apr}$ & 42.4 & 18.6 & 10.6 & 7 & 14 & 8 & 18 \\
\hline $23 \mathrm{Apr}$ & 42.1 & 4.6 & 11.1 & 23 & 43 & 24 & 45 \\
\hline $24 \mathrm{Apr}$ & 40.1 & 0.0 & 10.6 & 20 & 38 & 23 & 41 \\
\hline $25 \mathrm{Apr}$ & 41.5 & 0.0 & 12.2 & 34 & 47 & 41 & 60 \\
\hline $26 \mathrm{Apr}$ & 41.8 & 0.0 & 12.2 & 48 & 56 & 55 & 67 \\
\hline $27 \mathrm{Apr}$ & 45.6 & 0.0 & 12.2 & 22 & 24 & 27 & 33 \\
\hline $28 \mathrm{Apr}$ & 54.6 & 0.0 & 11.7 & 40 & 47 & 48 & 58 \\
\hline $29 \mathrm{Apr}$ & 54.1 & 0.0 & 11.7 & 34 & 39 & 40 & 49 \\
\hline $30 \mathrm{Apr}$ & 54.7 & 0.0 & 12.8 & 34 & 40 & 41 & 49 \\
\hline 01 May & 50.1 & 0.0 & 13.3 & 18 & 21 & 21 & 25 \\
\hline 02 May & 50.3 & 0.0 & 12.2 & 15 & 16 & 21 & 25 \\
\hline 03 May & 60.9 & 0.0 & 12.2 & 26 & 27 & 34 & 40 \\
\hline 04 May & 70.4 & 0.0 & 12.2 & 54 & 64 & 64 & 76 \\
\hline 05 May & 79.1 & 3.7 & 12.8 & 41 & 49 & 52 & 63 \\
\hline 06 May & 85.3 & 2.6 & 12.8 & 20 & 27 & 27 & 34 \\
\hline 07 May & 80.5 & 0.0 & 12.8 & 33 & 39 & 39 & 48 \\
\hline 08 May & 78.7 & 0.0 & 12.8 & 30 & 35 & 36 & 43 \\
\hline 09 May & 75.2 & 0.0 & 12.8 & 24 & 26 & 28 & 32 \\
\hline 10 May & 73.1 & 0.0 & 12.8 & 46 & 51 & 50 & 57 \\
\hline 11 May & 72.2 & 0.0 & 12.8 & 32 & 35 & 36 & 41 \\
\hline
\end{tabular}


Appendix Table 19. Continued.

\begin{tabular}{|c|c|c|c|c|c|c|c|}
\hline \multirow[b]{2}{*}{ Date } & \multirow[b]{2}{*}{$\begin{array}{l}\text { Average } \\
\text { flow } \\
\text { (kcfs) }\end{array}$} & \multirow[b]{2}{*}{$\begin{array}{c}\text { Average } \\
\text { spill } \\
\text { (kcfs) }\end{array}$} & \multirow[b]{2}{*}{$\begin{array}{l}\text { Scroll-case } \\
\text { water } \\
\text { temperature }\end{array}$} & \multicolumn{2}{|c|}{ Idaho only } & \multicolumn{2}{|c|}{ Idaho and Oregon } \\
\hline & & & & $\begin{array}{l}\text { Numbers } \\
\text { detected }\end{array}$ & $\begin{array}{l}\text { Expanded } \\
\text { numbers } \\
\text { detected }\end{array}$ & $\begin{array}{l}\text { Numbers } \\
\text { detected }\end{array}$ & $\begin{array}{c}\text { Expanded } \\
\text { numbers } \\
\text { detected }\end{array}$ \\
\hline 12 May & 72.1 & 0.0 & 12.2 & 13 & 14 & 13 & 15 \\
\hline 13 May & 64.1 & 0.0 & 12.2 & 10 & 10 & 11 & 12 \\
\hline 14 May & 59.5 & 0.0 & 12.2 & 17 & 17 & 18 & 20 \\
\hline 15 May & 57.9 & 0.0 & 11.7 & 19 & 21 & 24 & 28 \\
\hline 16 May & 55.9 & 0.0 & 11.7 & 17 & 19 & 19 & 22 \\
\hline 17 May & 68.2 & 0.0 & 12.8 & 10 & 11 & 11 & 13 \\
\hline 18 May & 64.9 & 0.0 & 12.8 & 17 & 19 & 22 & 25 \\
\hline 19 May & 67.7 & 0.0 & 13.3 & 12 & 12 & 17 & 19 \\
\hline 20 May & 75.5 & 0.0 & 13.3 & 12 & 12 & 12 & 13 \\
\hline 21 May & 77.6 & 0.0 & 13.3 & 22 & 23 & 24 & 27 \\
\hline 22 May & 83.6 & 0.0 & 13.3 & 20 & 22 & 26 & 30 \\
\hline 23 May & 84.6 & 0.0 & 13.3 & 17 & 19 & 20 & 23 \\
\hline 24 May & 89.7 & 0.0 & 13.3 & 18 & 19 & 20 & 25 \\
\hline 25 May & 86.7 & 0.0 & 12.8 & 8 & 8 & 13 & 16 \\
\hline 26 May & 84.2 & 0.0 & 12.8 & 14 & 18 & 16 & 21 \\
\hline 27 May & 92.8 & 4.4 & 12.8 & 11 & 15 & 15 & 21 \\
\hline 28 May & 122.4 & 29.9 & 12.8 & 12 & 21 & 14 & 23 \\
\hline 29 May & 132.4 & 39.4 & 12.2 & 3 & 6 & 4 & 7 \\
\hline 30 May & 125.8 & 34.2 & 11.7 & 6 & 10 & 7 & 12 \\
\hline 31 May & 120.8 & 28.4 & 11.1 & 4 & 8 & 7 & 13 \\
\hline 01 Jun & 114.8 & 22.2 & 11.7 & 2 & 4 & 2 & 5 \\
\hline 02 Jun & 103.1 & 11.4 & 11.7 & 1 & 2 & 1 & 2 \\
\hline 03 Jun & 100.4 & 18.9 & 11.7 & 2 & 4 & 2 & 5 \\
\hline 04 Jun & 105.1 & 22.5 & 11.7 & 3 & 7 & 3 & 7 \\
\hline 05 Jun & 108.9 & 19.0 & 14.4 & 3 & 6 & 4 & 8 \\
\hline 07 Jun & 110.6 & 21.7 & 14.4 & 1 & 2 & 2 & 3 \\
\hline 08 Jun & 102.0 & 9.5 & 13.9 & 2 & 4 & 3 & 5 \\
\hline 09 Jun & 97.6 & 5.6 & 13.9 & 4 & 7 & 5 & 8 \\
\hline 10 Jun & 90.5 & 0.0 & 13.9 & 5 & 6 & 6 & 8 \\
\hline 11 Jun & 88.1 & 0.0 & 13.9 & 3 & 3 & 5 & 6 \\
\hline 12 Jun & 83.8 & 0.0 & 13.3 & 3 & 3 & 3 & 4 \\
\hline 13 Jun & 77.2 & 0.0 & 13.3 & 2 & 2 & 3 & 4 \\
\hline 14 Jun & 76.5 & 0.0 & 13.9 & 3 & 3 & 3 & 4 \\
\hline 15 Jun & 72.6 & 0.0 & 14.4 & 2 & 2 & 4 & 5 \\
\hline 16 Jun & 60.7 & 0.0 & 14.4 & 1 & 1 & 1 & 1 \\
\hline 17 Jun & 55.2 & 0.0 & 16.1 & 3 & 3 & 3 & 4 \\
\hline 19 Jun & 49.3 & 0.0 & 17.2 & 1 & 1 & 1 & 1 \\
\hline 21 Jun & 46.2 & 0.0 & 17.8 & 1 & 1 & 2 & 2 \\
\hline 23 Jun & 48.4 & 0.0 & 17.8 & 1 & 1 & 1 & 1 \\
\hline 25 Jun & 52.9 & 0.0 & 17.8 & 0 & 0 & 1 & 1 \\
\hline 26 Jun & 43.6 & 0.0 & 18.9 & 1 & 1 & 1 & 1 \\
\hline $05 \mathrm{Jul}$ & 36.2 & 0.0 & 20.0 & 1 & 1 & 1 & 1 \\
\hline $12 \mathrm{Jul}$ & 37.8 & 0.0 & 20.0 & 1 & 1 & 1 & 1 \\
\hline $16 \mathrm{Jul}$ & 37.6 & 0.0 & 20.0 & 1 & 1 & 1 & 1 \\
\hline
\end{tabular}


Appendix Table 20. Daily first-time detections of PIT-tagged wild spring/summer Chinook salmon smolts from Idaho at Little Goose Dam during 2004, with associated river flows ( $\mathrm{kcfs}$ ), spill ( $\mathrm{kcfs}$ ), and water temperatures $\left({ }^{\circ} \mathrm{C}\right)$ at the dam.

\begin{tabular}{|c|c|c|c|c|}
\hline Date & $\begin{array}{l}\text { Average flow } \\
\text { (kcfs) }\end{array}$ & $\begin{array}{l}\text { Average spill } \\
\text { (kcfs) }\end{array}$ & $\begin{array}{l}\text { Scroll-case water } \\
\text { temperature }\end{array}$ & $\begin{array}{l}\text { Numbers } \\
\text { detected }\end{array}$ \\
\hline $13 \mathrm{Apr}$ & 57.0 & 20.6 & 10.9 & 3 \\
\hline $16 \mathrm{Apr}$ & 57.2 & 20.6 & 11.1 & 3 \\
\hline 17 Apr & 51.1 & 19.0 & 11.1 & 2 \\
\hline $18 \mathrm{Apr}$ & 51.4 & 20.1 & 11.1 & 7 \\
\hline 19 Apr & 48.4 & 18.3 & 11.1 & 13 \\
\hline $20 \mathrm{Apr}$ & 45.8 & 16.9 & 11.7 & 10 \\
\hline $21 \mathrm{Apr}$ & 45.3 & 15.0 & 11.7 & 6 \\
\hline $22 \mathrm{Apr}$ & 44.7 & 15.6 & 11.7 & 2 \\
\hline $23 \mathrm{Apr}$ & 42.6 & 7.4 & 11.7 & 12 \\
\hline 24 Apr & 40.0 & 0.0 & 11.7 & 23 \\
\hline $25 \mathrm{Apr}$ & 41.7 & 0.0 & 11.7 & 19 \\
\hline $26 \mathrm{Apr}$ & 41.9 & 0.0 & 11.7 & 9 \\
\hline $27 \mathrm{Apr}$ & 44.6 & 0.0 & 11.0 & 14 \\
\hline $28 \mathrm{Apr}$ & 53.4 & 0.0 & 11.0 & 18 \\
\hline 29 Apr & 54.4 & 0.0 & 11.1 & 6 \\
\hline $30 \mathrm{Apr}$ & 54.0 & 0.0 & 11.7 & 1 \\
\hline 01 May & 48.9 & 0.0 & 11.7 & 1 \\
\hline 02 May & 50.5 & 0.0 & 11.7 & 5 \\
\hline 03 May & 61.7 & 0.0 & 11.7 & 1 \\
\hline 04 May & 70.7 & 0.0 & 11.7 & 2 \\
\hline 05 May & 77.8 & 0.0 & 11.7 & 3 \\
\hline 06 May & 85.4 & 0.0 & 11.7 & 6 \\
\hline 07 May & 81.1 & 0.0 & 11.7 & 8 \\
\hline 08 May & 78.3 & 0.0 & 11.7 & 4 \\
\hline 09 May & 73.5 & 0.0 & 11.7 & 1 \\
\hline 10 May & 73.8 & 0.0 & 11.7 & 5 \\
\hline 11 May & 71.4 & 0.0 & 12.8 & 3 \\
\hline 12 May & 72.0 & 0.0 & 12.8 & 4 \\
\hline 13 May & 65.2 & 0.0 & 12.8 & 1 \\
\hline 14 May & 58.5 & 0.0 & 12.8 & 2 \\
\hline 15 May & 58.4 & 0.0 & 12.8 & 3 \\
\hline 16 May & 56.3 & 0.0 & 12.8 & 2 \\
\hline 17 May & 68.7 & 0.0 & 12.8 & 2 \\
\hline 18 May & 64.4 & 0.0 & 12.8 & 4 \\
\hline
\end{tabular}


Appendix Table 20. Continued.

\begin{tabular}{|c|c|c|c|c|}
\hline Date & $\begin{array}{l}\text { Average flow } \\
\quad(\mathrm{kcfs})\end{array}$ & $\begin{array}{l}\text { Average spill } \\
\quad(\mathrm{kcfs})\end{array}$ & $\begin{array}{l}\text { Scroll-case water } \\
\text { temperature }\end{array}$ & $\begin{array}{l}\text { Numbers } \\
\text { detected }\end{array}$ \\
\hline 17 May & 68.7 & 0.0 & 12.8 & 2 \\
\hline 18 May & 64.4 & 0.0 & 12.8 & 4 \\
\hline 21 May & 78.2 & 0.0 & 12.2 & 1 \\
\hline 22 May & 83.5 & 0.0 & 12.2 & 1 \\
\hline 23 May & 83.0 & 0.0 & 12.2 & 2 \\
\hline 24 May & 89.9 & 0.0 & 12.8 & 1 \\
\hline 25 May & 87.4 & 0.0 & 12.8 & 1 \\
\hline 26 May & 84.8 & 0.0 & 13.3 & 2 \\
\hline 27 May & 93.7 & 0.0 & 13.3 & 1 \\
\hline 28 May & 117.3 & 4.4 & 13.3 & 1 \\
\hline 30 May & 124.3 & 12.7 & 13.3 & 2 \\
\hline 31 May & 119.2 & 5.5 & 13.3 & 2 \\
\hline 01 June & 115.0 & 4.5 & 13.3 & 2 \\
\hline 02 June & 105.1 & 3.5 & 13.3 & 2 \\
\hline 03 June & 100.3 & 0.0 & 13.3 & 2 \\
\hline 04 June & 104.1 & 0.0 & 13.3 & 1 \\
\hline 05 June & 109.5 & 0.9 & 13.3 & 6 \\
\hline 06 June & 108.2 & 0.0 & 13.9 & 8 \\
\hline 07 June & 111.2 & 0.0 & 13.9 & 3 \\
\hline 09 June & 102.0 & 0.0 & 15.0 & 2 \\
\hline 12 June & 84.3 & 0.0 & 14.4 & 2 \\
\hline 13 June & 76.3 & 0.0 & 14.4 & 2 \\
\hline 14 June & 75.7 & 0.0 & 14.4 & 2 \\
\hline 16 June & 60.3 & 0.0 & 14.4 & 1 \\
\hline 18 June & 54.2 & 0.0 & 14.4 & 1 \\
\hline
\end{tabular}


Appendix Table 21. Daily first-time detections of PIT-tagged wild spring/summer Chinook salmon smolts from Idaho at Lower Monumental Dam during 2004, with associated river flows (kcfs), spill (kcfs), and water temperatures $\left({ }^{\circ} \mathrm{C}\right)$ at the dam.

\begin{tabular}{lcccc}
\hline Date & $\begin{array}{c}\text { Average flow } \\
(\mathrm{kcfs})\end{array}$ & $\begin{array}{c}\text { Average spill } \\
(\mathrm{kcfs})\end{array}$ & $\begin{array}{c}\text { Scroll-case water } \\
\text { temperature }\end{array}$ & $\begin{array}{c}\text { Numbers } \\
\text { detected }\end{array}$ \\
\hline $15 \mathrm{Apr}$ & 69.8 & 0.0 & 11.1 & 1 \\
$17 \mathrm{Apr}$ & 50.6 & 0.0 & 11.1 & 2 \\
$20 \mathrm{Apr}$ & 46.2 & 0.0 & 11.7 & 2 \\
$21 \mathrm{Apr}$ & 46.2 & 0.0 & 11.7 & 6 \\
$22 \mathrm{Apr}$ & 46.3 & 0.0 & 11.7 & 7 \\
$23 \mathrm{Apr}$ & 42.0 & 0.0 & 11.7 & 7 \\
$24 \mathrm{Apr}$ & 42.3 & 4.5 & 11.7 & 5 \\
$25 \mathrm{Apr}$ & 45.3 & 11.6 & 11.7 & 2 \\
$27 \mathrm{Apr}$ & 46.5 & 8.0 & 11.7 & 1 \\
$28 \mathrm{Apr}$ & 10.9 & 11.1 & 1 \\
$29 \mathrm{Apr}$ & 55.1 & 13.8 & 11.1 & 1 \\
$14 \mathrm{May}$ & 56.9 & 5.9 & 12.8 & 1 \\
$17 \mathrm{May}$ & 60.0 & 0.0 & 12.8 & 1 \\
$21 \mathrm{May}$ & 72.8 & 0.0 & 12.2 & 1 \\
$25 \mathrm{May}$ & 83.1 & 0.0 & 13.3 & 2 \\
$28 \mathrm{May}$ & 94.0 & 3.9 & 13.3 & 1 \\
$30 \mathrm{May}$ & 124.2 & 14.6 & 12.8 & 1 \\
07 Jun & 130.1 & 6.0 & 12.8 & 1 \\
$13 \mathrm{Jun}$ & 118.4 & 0.0 & 14.4 & 18.9 \\
$29 \mathrm{Jun}$ & 79.0 & 0.0 & & \\
& 38.6 & & & 1 \\
\hline & & & & 1 \\
\hline
\end{tabular}


Appendix Table 22. Daily first-time detections of PIT-tagged wild spring/summer

Chinook salmon smolts from Idaho at McNary Dam during 2004, with associated river flows (kcfs), spill ( $\mathrm{kcfs}$ ), and water temperatures $\left({ }^{\circ} \mathrm{C}\right)$ at the dam. Two first-time detections occurred at John Day Dam: the first was on 5 May for a fish tagged at Elk Creek; the second was on 20 May for a fish tagged at Loon Creek. Two first-time detections also occurred at Bonneville dam: the first was on 15 May for a fish tagged at Herd Creek; the second was on 23 May for a fish tagged at West Fork Chamberlain Creek.

\begin{tabular}{lcccc}
\hline Date & $\begin{array}{c}\text { Average flow } \\
(\mathrm{kcfs})\end{array}$ & $\begin{array}{c}\text { Average spill } \\
(\mathrm{kcfs})\end{array}$ & $\begin{array}{c}\text { Scroll-case water } \\
\text { temperature }\end{array}$ & $\begin{array}{c}\text { Numbers } \\
\text { detected }\end{array}$ \\
\hline $24 \mathrm{Apr}$ & 155.0 & 39.1 & 10.9 & 1 \\
$26 \mathrm{Apr}$ & 154.1 & 37.3 & 11.6 & 1 \\
$27 \mathrm{Apr}$ & 179.3 & 55.5 & 11.3 & 1 \\
$28 \mathrm{Apr}$ & 179.3 & 55.5 & 11.3 & 3 \\
29 Apr & 175.4 & 58.0 & 12.1 & 2 \\
30 Apr & 166.5 & 55.1 & 11.9 & 2 \\
01 May & 164.7 & 52.1 & 12.3 & 4 \\
02 May & 164.5 & 52.9 & 12.3 & 1 \\
03 May & 170.2 & 57.4 & 12.5 & 2 \\
04 May & 206.9 & 87.2 & 12.8 & 1 \\
05 May & 226.0 & 98.7 & 12.7 & 2 \\
07 May & 211.6 & 81.8 & 12.8 & 2 \\
08 May & 202.2 & 87.3 & 12.7 & 1 \\
03 Jun & 232.8 & 78.1 & 15.1 & \\
\hline
\end{tabular}


Appendix Table 23. Monthly environmental data collected from Marsh Creek (rkm 179.5 from the mouth of the Middle Fork Salmon River) from August 2003 through July 2004.

\begin{tabular}{|c|c|c|c|c|c|c|c|c|c|c|c|c|}
\hline & Aug & Sep & Oct & Nov & Dec & Jan & Feb & Mar & Apr & May & Jun & Jul \\
\hline \multicolumn{13}{|c|}{ Temperature $\left({ }^{\circ} \mathrm{C}\right)$} \\
\hline Min & 6.9 & 2.5 & 0.1 & 0.0 & 0.0 & 0.0 & 0.0 & 0.0 & 0.1 & 1.6 & 3.6 & 6.9 \\
\hline Max & 15.9 & 15.9 & 12.6 & 3.4 & 4.5 & 3.0 & 5.3 & 8.8 & 12.0 & 12.6 & 15.9 & 15.9 \\
\hline Mean & 11.3 & 8.8 & 5.6 & 0.7 & 0.7 & 0.4 & 0.9 & 2.9 & 4.2 & 6.4 & 9.4 & 11.6 \\
\hline \multicolumn{13}{|c|}{ Dissolved Oxygen (ppm) } \\
\hline Min & --- & --- & --- & --- & 11.4 & 11.0 & 10.5 & 10.0 & 9.1 & --- & --- & --- \\
\hline Max & --- & --- & --- & --- & 14.2 & 13.1 & 13.1 & 12.6 & 12.1 & --- & --- & --- \\
\hline Mean & --- & --- & --- & --- & 12.7 & 11.7 & 11.7 & 11.4 & 10.7 & --- & --- & --- \\
\hline \multicolumn{13}{|c|}{ Specific Conductance $(\mu \mathrm{S} / \mathrm{cm})$} \\
\hline Min & 65.0 & 69.0 & 73.0 & 63.0 & 49.0 & 44.0 & 53.0 & 53.0 & 35.0 & 20.0 & 21.0 & 14.0 \\
\hline Max & 74.0 & 81.0 & 79.0 & 85.0 & 72.0 & 68.0 & 68.0 & 68.0 & 55.0 & 48.0 & 31.0 & 35.0 \\
\hline Mean & 70.5 & 72.9 & 75.8 & 72.6 & 63.7 & 63.0 & 64.6 & 62.5 & 46.5 & 30.0 & 25.7 & 28.5 \\
\hline \multicolumn{13}{|c|}{ Turbidity (ntu) } \\
\hline Min & 0.2 & 0.1 & 0.0 & 0.0 & 0.0 & 0.0 & 0.0 & 0.5 & 1.3 & 1.2 & 14.7 & --- \\
\hline Max & 31.9 & 41.4 & 1.3 & 3.7 & 2.1 & 49.7 & 49.7 & 49.8 & 23.6 & 36.9 & 49.4 & --- \\
\hline Mean & 0.8 & 2.0 & 0.4 & 0.3 & 0.2 & 4.4 & 8.6 & 15.5 & 4.6 & 6.4 & 34.5 & --- \\
\hline \multicolumn{13}{|c|}{ Depth (ft) } \\
\hline Min & 1.0 & 0.7 & 0.3 & 0.3 & 0.3 & 0.3 & 0.3 & 0.4 & 1.1 & 1.9 & 2.0 & 1.5 \\
\hline Max & 1.4 & 1.3 & 1.4 & 1.9 & 1.5 & 2.2 & 1.7 & 1.4 & 2.1 & 2.8 & 2.8 & 2.1 \\
\hline Mean & 1.2 & 1.0 & 1.0 & 0.9 & 0.9 & 1.3 & 0.8 & 0.9 & 1.7 & 2.4 & 2.3 & 1.8 \\
\hline \multicolumn{13}{|c|}{ pH } \\
\hline Min & 7.3 & 7.4 & 7.4 & 7.4 & 7.4 & 7.2 & 7.4 & 7.1 & 6.9 & 7.1 & 7.3 & 7.2 \\
\hline Max & 8.9 & 9.0 & 9.1 & 8.4 & 8.5 & 8.2 & 8.7 & 7.9 & 8.3 & 7.9 & 8.4 & 8.4 \\
\hline Mean & 7.8 & 7.9 & 7.9 & 7.7 & 7.6 & 7.5 & 7.6 & 7.5 & 7.3 & 7.3 & 7.6 & 7.6 \\
\hline
\end{tabular}


Appendix Table 24. Monthly environmental data collected from the Salmon River near Sawtooth Hatchery (rkm 627.9) from August 2003 through July 2004.

\begin{tabular}{|c|c|c|c|c|c|c|c|c|c|c|c|c|}
\hline & Aug & Sep & Oct & Nov & Dec & Jan & Feb & Mar & Apr & May & Jun & Jul \\
\hline \multicolumn{13}{|c|}{ Temperature $\left({ }^{\circ} \mathbf{C}\right)$} \\
\hline Min & 9.2 & 5.3 & 1.4 & 0.0 & --- & --- & --- & --- & 2.7 & 5.0 & 7.4 & 9.9 \\
\hline Max & 15.9 & 15.9 & 13.9 & 5.7 & --- & --- & --- & --- & 14.1 & 15.0 & 15.9 & 15.9 \\
\hline Mean & 13.1 & 10.9 & 7.9 & 2.2 & --- & --- & --- & --- & 7.3 & 9.7 & 12.2 & 13.3 \\
\hline \multicolumn{13}{|c|}{ Dissolved Oxygen (ppm) } \\
\hline Min & 6.6 & 8.0 & --- & 11.2 & --- & --- & --- & --- & 7.1 & 7.3 & 6.2 & 7.6 \\
\hline Max & 10.4 & 12.0 & --- & 14.2 & --- & --- & --- & --- & 10.6 & 12.6 & 11.3 & 11.2 \\
\hline Mean & 8.8 & 9.7 & --- & 12.4 & --- & --- & --- & --- & 8.6 & 10.0 & 8.9 & 8.9 \\
\hline \multicolumn{13}{|c|}{ Specific Conductance $(\mu \mathrm{S} / \mathrm{cm})$} \\
\hline Min & 136.0 & 140.0 & 144.0 & 135.0 & --- & --- & --- & --- & 94.0 & 69.0 & 65.0 & 85.0 \\
\hline Max & 152.0 & 158.0 & 158.0 & 159.0 & --- & --- & --- & --- & 122.0 & 101.0 & 89.0 & 126.0 \\
\hline Mean & 143.5 & 152.6 & 151.5 & 145.8 & --- & --- & --- & --- & 105.4 & 84.1 & 80.3 & 108.0 \\
\hline \multicolumn{13}{|c|}{ Turbidity (ntu) } \\
\hline Min & 0.0 & 0.0 & 0.0 & 0.0 & --- & --- & --- & -- & 1.0 & 1.7 & 1.3 & 0.3 \\
\hline Max & 1.0 & 13.6 & 32.5 & 42.5 & --- & --- & --- & --- & 49.1 & 39.4 & 27.8 & 2.9 \\
\hline Mean & 0.3 & 0.5 & 0.3 & 1.1 & --- & -- & --- & -- & 3.0 & 5.4 & 4.6 & 1.0 \\
\hline \multicolumn{13}{|c|}{ Depth (feet) } \\
\hline Min & 1.6 & 1.3 & 0.9 & 0.9 & --- & --- & --- & --- & 1.6 & 1.9 & 2.2 & 1.9 \\
\hline Max & 2.1 & 1.9 & 2.2 & 1.9 & --- & --- & --- & --- & 2.3 & 2.6 & 2.6 & 2.5 \\
\hline Mean & 1.8 & 1.7 & 1.7 & 1.4 & --- & --- & --- & --- & 2.0 & 2.2 & 2.4 & 2.1 \\
\hline \multicolumn{13}{|c|}{ pH } \\
\hline Min & 7.9 & 8.0 & 8.0 & 7.9 & --- & --- & --- & --- & 7.7 & 7.6 & 7.6 & 7.6 \\
\hline Max & 8.8 & 9.1 & 9.2 & 9.1 & --- & --- & --- & --- & 8.3 & 8.5 & 8.7 & 8.9 \\
\hline Mean & 8.3 & 8.4 & 8.4 & 8.3 & --- & --- & --- & --- & 8.0 & 8.0 & 8.0 & 8.1 \\
\hline
\end{tabular}


Appendix Table 25. Monthly environmental data collected from Valley Creek (rkm 609.4 from the mouth of the Salmon River) from August 2003 through July 2004.

\begin{tabular}{|c|c|c|c|c|c|c|c|c|c|c|c|c|}
\hline & Aug & Sep & Oct & Nov & Dec & Jan & Feb & Mar & Apr & May & Jun & Jul \\
\hline \multicolumn{13}{|c|}{ Temperature $\left({ }^{\circ} \mathrm{C}\right)$} \\
\hline Min & 9.3 & 3.2 & 0.6 & 0.0 & 0.0 & 0.0 & 0.1 & 0.0 & 1.7 & 4.0 & 6.8 & 9.0 \\
\hline Max & 15.9 & 15.9 & 15.0 & 4.6 & 3.2 & 1.1 & 1.8 & 9.8 & 14.7 & 15.0 & 15.9 & 15.9 \\
\hline Mean & 13.2 & 10.7 & 7.2 & 1.0 & 0.9 & 0.5 & 0.6 & 3.0 & 6.7 & 8.9 & 11.6 & 13.2 \\
\hline \multicolumn{13}{|c|}{ Dissolved Oxygen (ppm) } \\
\hline Min & --- & --- & --- & 8.3 & 8.6 & 12.7 & 13.2 & --- & --- & --- & --- & --- \\
\hline Max & --- & --- & --- & 9.6 & 14.1 & 14.1 & 14.2 & --- & --- & --- & --- & --- \\
\hline Mean & --- & --- & --- & 9.1 & 12.7 & 13.3 & 13.8 & --- & --- & --- & --- & --- \\
\hline \multicolumn{13}{|c|}{ Specific Conductance $(\mu \mathrm{S} / \mathrm{cm})$} \\
\hline Min & 59.0 & 78.0 & 74.0 & 82.0 & 73.0 & 81.0 & 83.0 & 62.0 & 50.0 & 40.0 & 43.0 & 48.0 \\
\hline Max & 87.0 & 88.0 & 90.0 & 102.0 & 95.0 & 92.0 & 98.0 & 99.0 & 63.0 & 58.0 & 51.0 & 70.0 \\
\hline Mean & 75.6 & 82.7 & 82.4 & 88.5 & 83.3 & 86.2 & 90.8 & 85.1 & 57.0 & 47.1 & 47.3 & 59.3 \\
\hline \multicolumn{13}{|c|}{ Turbidity (ntu) } \\
\hline Min & 0.5 & 0.3 & 0.2 & 0.1 & 0.0 & 0.0 & 0.4 & 0.4 & 1.3 & 1.6 & 1.3 & 0.8 \\
\hline Max & 30.7 & 2.2 & 2.7 & 14.6 & 12.4 & 5.8 & 8.8 & 38.2 & 34.8 & 19.9 & 20.5 & 47.5 \\
\hline Mean & 1.6 & 0.9 & 0.7 & 1.2 & 0.9 & 0.8 & 1.3 & 3.9 & 4.0 & 4.6 & 3.1 & 3.2 \\
\hline \multicolumn{13}{|c|}{ Depth (ft) } \\
\hline Min & 1.1 & 0.8 & 0.5 & 0.6 & 0.4 & 0.2 & 0.5 & 0.5 & 1.2 & 1.6 & 1.9 & 1.5 \\
\hline $\operatorname{Max}$ & 1.6 & 1.4 & 1.6 & 1.6 & 1.4 & 1.3 & 1.5 & 1.6 & 2.0 & 2.4 & 2.4 & 2.1 \\
\hline Mean & 1.3 & 1.2 & 1.2 & 1.0 & 0.9 & 1.0 & 0.9 & 1.2 & 1.6 & 2.0 & 2.1 & 1.7 \\
\hline \multicolumn{13}{|c|}{ pH } \\
\hline Min & 7.3 & 7.7 & 7.8 & 7.7 & 7.5 & 7.4 & 7.5 & 7.4 & 7.4 & 7.2 & 7.3 & 7.4 \\
\hline Max & 8.4 & 8.6 & 8.7 & 8.4 & 8.2 & 8.1 & 8.2 & 8.2 & 8.4 & 8.2 & 8.5 & 8.4 \\
\hline Mean & 7.9 & 8.0 & 8.0 & 7.9 & 7.8 & 7.6 & 7.7 & 7.8 & 7.8 & 7.6 & 7.7 & 7.8 \\
\hline
\end{tabular}


Appendix Table 26. Monthly environmental data collected from Secesh River $(27 \mathrm{~km}$ upstream from its confluence with the South Fork Salmon River) from August 2003 through July 2004.

\begin{tabular}{|c|c|c|c|c|c|c|c|c|c|c|c|c|}
\hline & Aug & Sep & Oct & Nov & Dec & Jan & Feb & Mar & Apr & May & Jun & Jul \\
\hline \multicolumn{13}{|c|}{ Temperature $\left({ }^{\circ} \mathrm{C}\right)$} \\
\hline Min & --- & --- & --- & 0.0 & 0.1 & 0.1 & 0.1 & 0.0 & 0.4 & 0.9 & 4.5 & --- \\
\hline Max & --- & --- & --- & 0.2 & 0.1 & 0.1 & 0.1 & 6.3 & 8.8 & 9.1 & 15.1 & --- \\
\hline Mean & --- & --- & --- & 0.1 & 0.1 & 0.1 & 0.1 & 0.4 & 3.6 & 5.2 & 8.9 & --- \\
\hline \multicolumn{13}{|c|}{ Dissolved Oxygen (ppm) } \\
\hline Min & --- & --- & --- & 10.3 & 10.4 & 10.3 & 10.4 & 9.8 & 9.0 & 8.5 & 7.4 & --- \\
\hline Max & --- & --- & --- & 11.5 & 11.1 & 11.0 & 10.9 & 11.3 & 11.1 & 10.6 & 9.4 & --- \\
\hline Mean & --- & --- & --- & 10.7 & 10.7 & 10.6 & 10.6 & 10.5 & 10.3 & 9.5 & 8.6 & --- \\
\hline \multicolumn{13}{|c|}{ Specific Conductance $(\mu \mathrm{S} / \mathrm{cm})$} \\
\hline Min & --- & --- & --- & 32.0 & 32.0 & 34.0 & 34.0 & 26.0 & 20.0 & 17.0 & 18.0 & --- \\
\hline Max & --- & --- & --- & 41.0 & 36.0 & 36.0 & 36.0 & 36.0 & 29.0 & 23.0 & 27.0 & --- \\
\hline Mean & --- & --- & --- & 35.4 & 34.3 & 35.4 & 35.9 & 32.6 & 24.0 & 19.5 & 21.5 & --- \\
\hline \multicolumn{13}{|c|}{ Turbidity (ntu) } \\
\hline Min & --- & --- & --- & 0.0 & 0.1 & 0.1 & 0.1 & 0.2 & 0.8 & 1.1 & 0.9 & --- \\
\hline $\operatorname{Max}$ & --- & --- & --- & 3.2 & 4.0 & 0.6 & 2.0 & 14.0 & 11.4 & 46.4 & 15.6 & --- \\
\hline Mean & --- & --- & --- & 0.7 & 0.9 & 0.3 & 0.5 & 1.8 & 2.8 & 6.1 & 2.7 & --- \\
\hline \multicolumn{13}{|c|}{ Depth (ft) } \\
\hline Min & --- & --- & --- & 1.4 & 1.6 & 1.8 & 2.2 & 1.7 & 1.9 & 2.6 & 2.3 & --- \\
\hline Max & --- & --- & --- & 2.5 & 2.7 & 2.9 & 3.3 & 3.7 & 3.0 & 3.7 & 3.6 & --- \\
\hline Mean & --- & --- & --- & 1.9 & 2.1 & 2.5 & 2.9 & 3.0 & 2.4 & 3.1 & 2.9 & --- \\
\hline \multicolumn{13}{|c|}{ pH } \\
\hline Min & --- & --- & --- & 7.2 & 7.1 & 7.1 & 6.9 & 6.9 & 7.0 & 6.8 & 6.7 & --- \\
\hline Max & --- & --- & --- & 7.6 & 7.3 & 7.2 & 7.1 & 7.2 & 7.4 & 7.4 & 7.5 & --- \\
\hline Mean & --- & --- & --- & 7.3 & 7.2 & 7.1 & 7.0 & 7.1 & 7.1 & 7.0 & 7.0 & --- \\
\hline
\end{tabular}


Appendix Table 27. Monthly environmental data collected from South Fork Salmon River (112 km from its confluence with the Salmon River) from August 2003 through July 2004.

\begin{tabular}{|c|c|c|c|c|c|c|c|c|c|c|c|c|}
\hline & Aug & Sep & Oct & Nov & Dec & Jan & Feb & Mar & Apr & May & Jun & Jul \\
\hline \multicolumn{13}{|c|}{ Temperature $\left({ }^{\circ} \mathrm{C}\right)$} \\
\hline Min & --- & 4.1 & 0.0 & 0.0 & 0.0 & 0.0 & 0.0 & 0.0 & 0.8 & 2.3 & 0.0 & 8.1 \\
\hline Max & --- & 15.9 & 12.4 & 1.6 & 2.0 & 1.2 & 3.0 & 6.7 & 9.3 & 9.0 & 15.4 & 15.9 \\
\hline Mean & --- & 9.9 & 6.0 & 0.3 & 0.4 & 0.1 & 0.4 & 2.0 & 4.2 & 5.2 & 8.4 & 13.2 \\
\hline \multicolumn{13}{|c|}{ Dissolved Oxygen (ppm) } \\
\hline Min & --- & --- & 8.9 & 8.4 & 9.2 & 1.1 & --- & --- & 8.4 & 11.0 & --- & --- \\
\hline Max & --- & --- & 14.2 & 14.1 & 11.9 & 10.9 & --- & --- & 13.7 & 14.2 & --- & --- \\
\hline Mean & --- & --- & 13.7 & 11.5 & 10.5 & 9.4 & --- & --- & 11.2 & 13.2 & --- & --- \\
\hline \multicolumn{13}{|c|}{ Specific Conductance $(\mu \mathrm{S} / \mathrm{cm})$} \\
\hline Min & --- & 52.0 & 57.0 & 47.0 & 43.0 & 42.0 & 45.0 & 38.0 & 33.0 & 24.0 & 23.0 & 25.0 \\
\hline Max & --- & 61.0 & 76.0 & 82.0 & 63.0 & 63.0 & 65.0 & 63.0 & 46.0 & 35.0 & 35.0 & 47.0 \\
\hline Mean & --- & 56.8 & 65.2 & 58.6 & 56.1 & 57.5 & 58.3 & 50.7 & 38.8 & 27.9 & 29.1 & 37.8 \\
\hline \multicolumn{13}{|c|}{ Turbidity (ntu) } \\
\hline Min & --- & --- & 0.0 & 0.0 & 0.0 & 0.0 & 0.0 & 0.0 & 1.4 & 1.2 & 0.5 & 0.0 \\
\hline Max & --- & --- & 49.7 & 46.3 & 11.7 & 4.4 & 3.4 & 13.6 & 8.7 & 19.5 & 14.6 & 43.2 \\
\hline Mean & --- & --- & 6.9 & 0.6 & 0.3 & 0.1 & 0.2 & 1.7 & 2.8 & 3.9 & 2.2 & 1.5 \\
\hline \multicolumn{13}{|c|}{ Depth (ft) } \\
\hline Min & --- & 0.7 & 0.4 & 0.4 & 0.3 & 0.3 & 0.4 & 0.4 & 1.2 & 1.6 & 1.6 & 1.0 \\
\hline Max & --- & 1.3 & 1.5 & 2.0 & 1.8 & 2.4 & 1.9 & 1.6 & 2.0 & 2.5 & 2.5 & 2.5 \\
\hline Mean & --- & 1.0 & 1.0 & 1.1 & 1.0 & 1.4 & 0.9 & 1.1 & 1.6 & 2.0 & 1.9 & 1.4 \\
\hline \multicolumn{13}{|c|}{$\mathbf{p H}$} \\
\hline Min & --- & 7.3 & 7.4 & 7.3 & 7.5 & 7.7 & 7.6 & 7.3 & 7.3 & 7.1 & 7.1 & 6.9 \\
\hline Max & --- & 8.7 & 8.5 & 8.4 & 8.0 & 8.1 & 8.6 & 8.4 & 7.8 & 7.6 & 8.1 & 8.3 \\
\hline Mean & --- & 7.7 & 7.6 & 7.7 & 7.7 & 7.7 & 7.8 & 7.6 & 7.4 & 7.3 & 7.4 & 7.6 \\
\hline
\end{tabular}


Upper Salmon River Trap
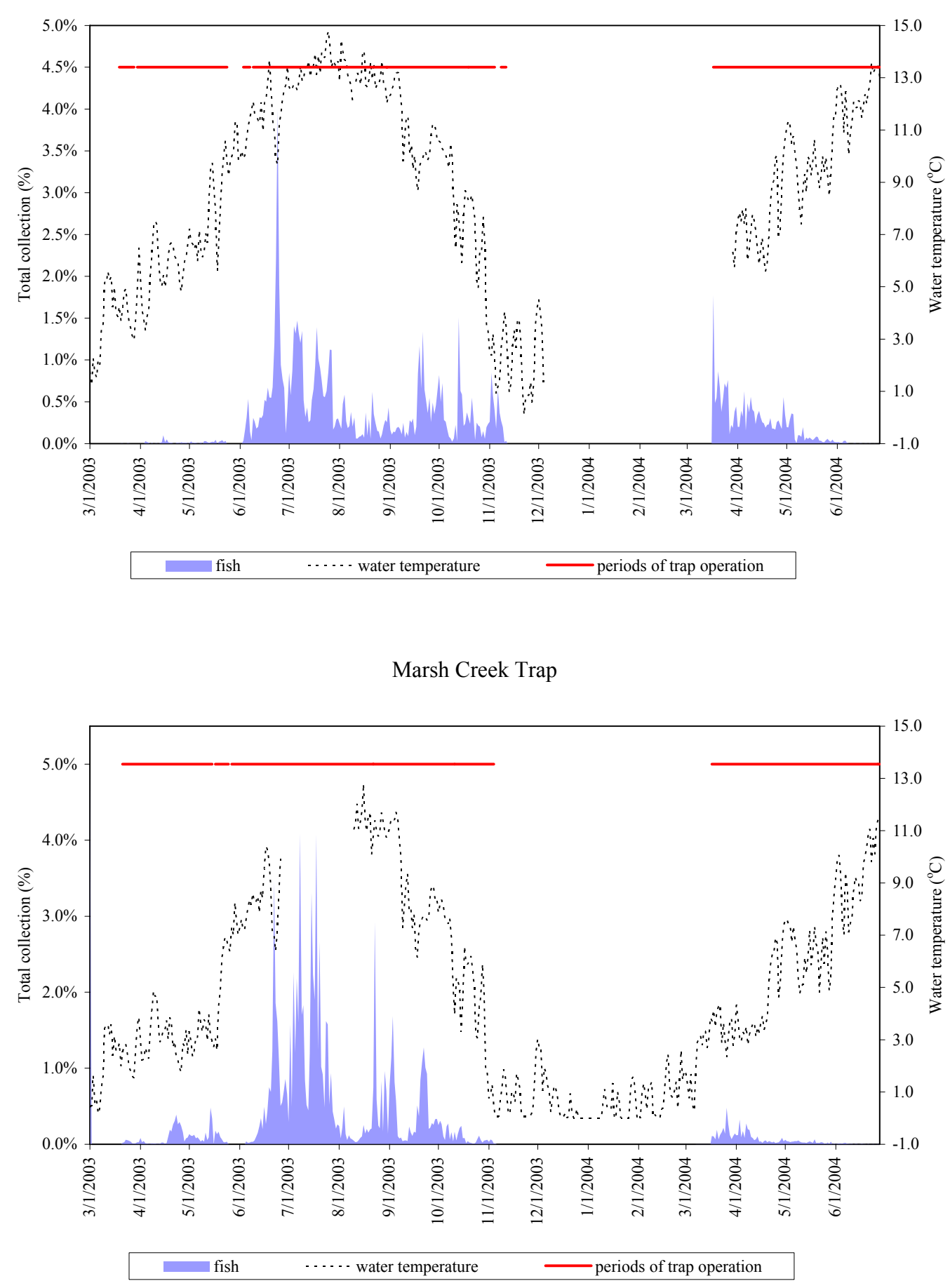

Appendix Figure 1. Daily passage of wild Chinook salmon fry, parr, and smolts at four migrant traps, expressed as percentages of total collected, and plotted against average daily water temperatures collected near traps. Periods of trap operation are also shown. 
South Fork Salmon River Trap

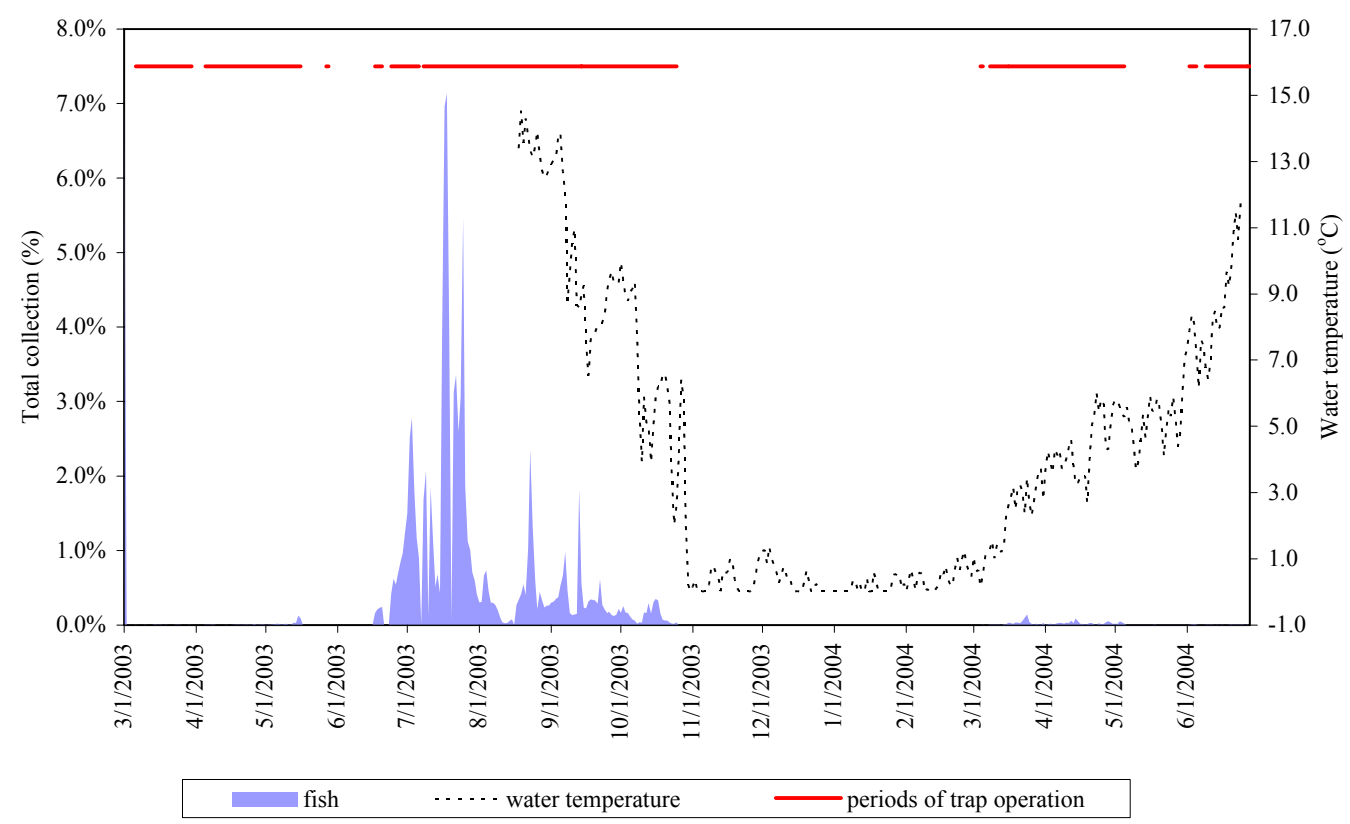

Secesh River Trap

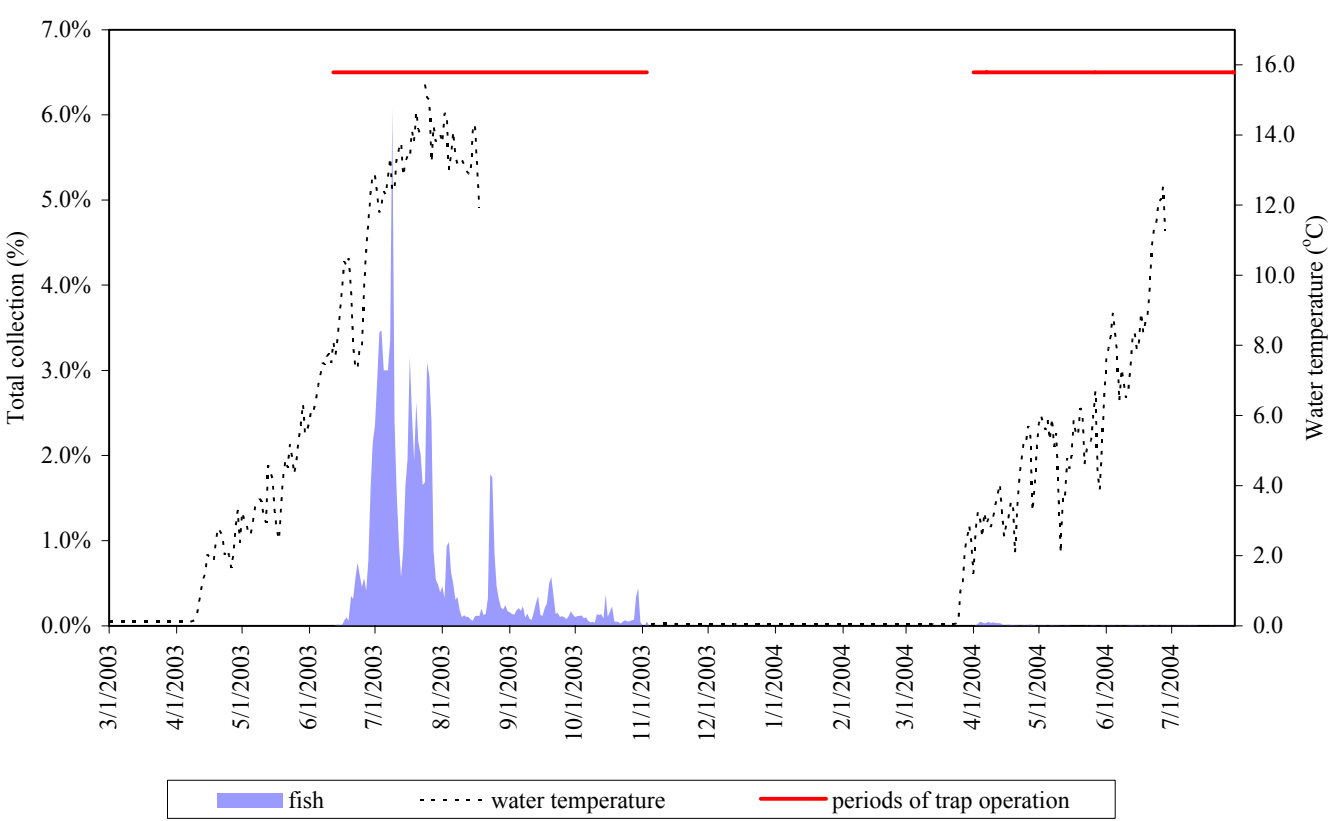

Appendix Figure 1. Continued. 
Upper Salmon River Trap

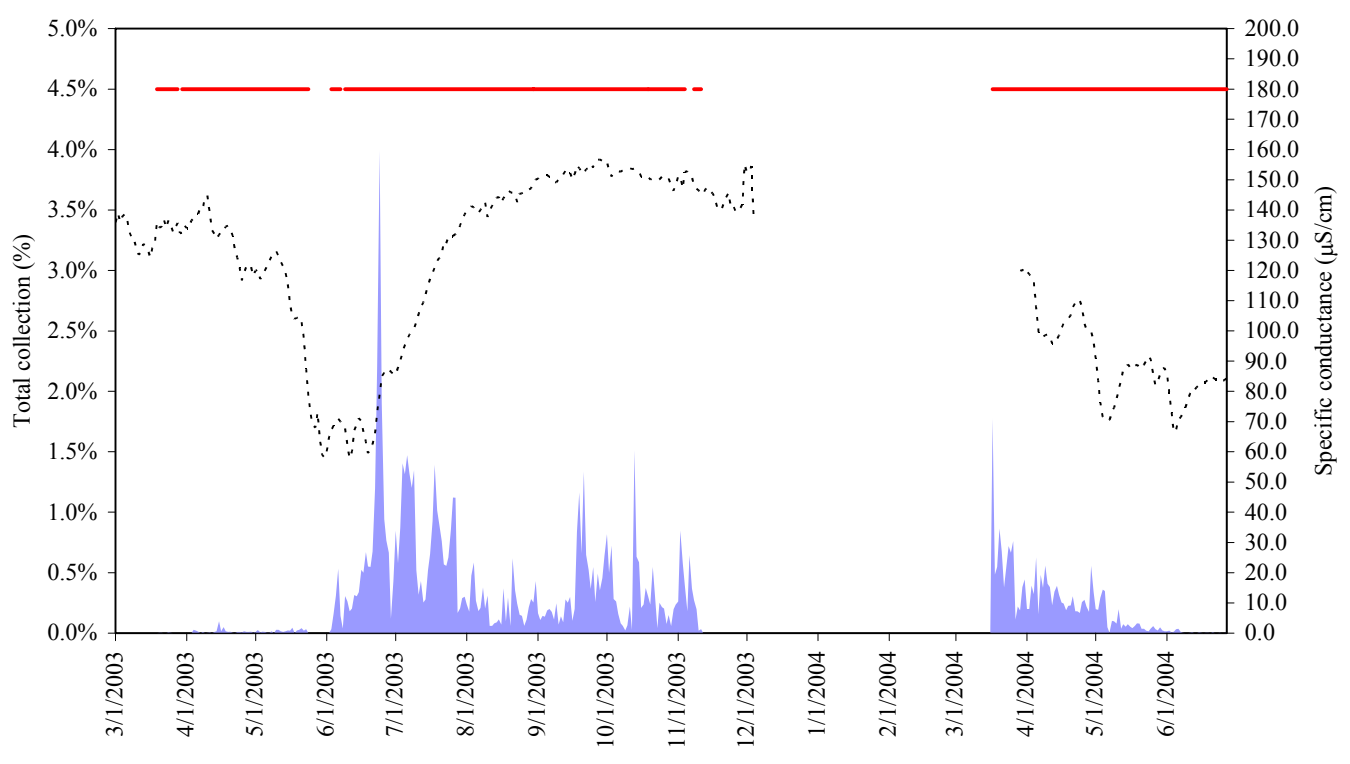

\begin{tabular}{|lll|}
\hline fish & $\cdots \cdots$ specific conductance $\quad-$ periods of trap operation \\
\hline
\end{tabular}

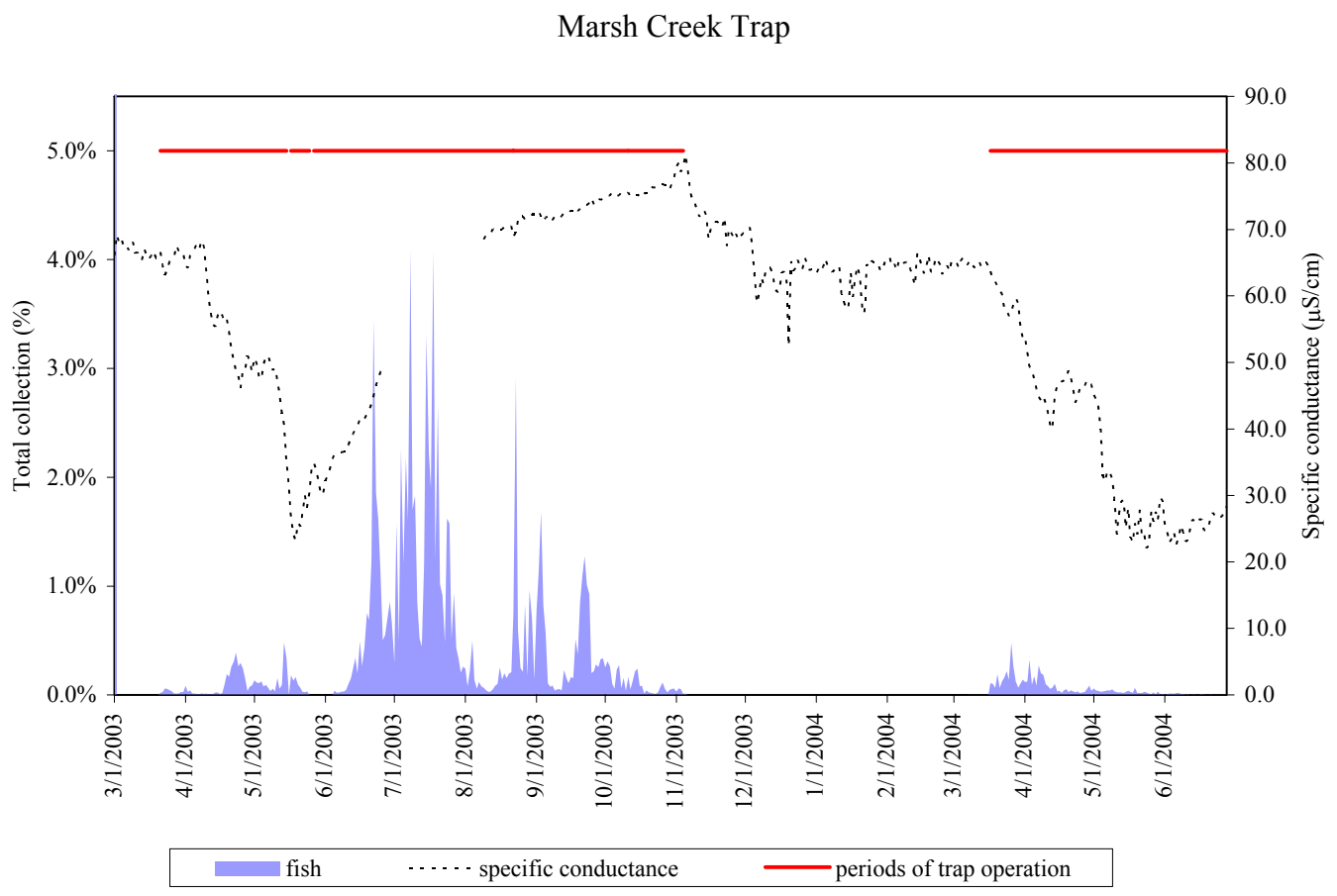

Appendix Figure 2. Daily passage of wild Chinook salmon fry, parr, and smolts at four migrant traps, expressed as percentages of total collected, and plotted against average daily specific conductance collected near traps. Periods of trap operation are also shown. 
South Fork Salmon River Trap

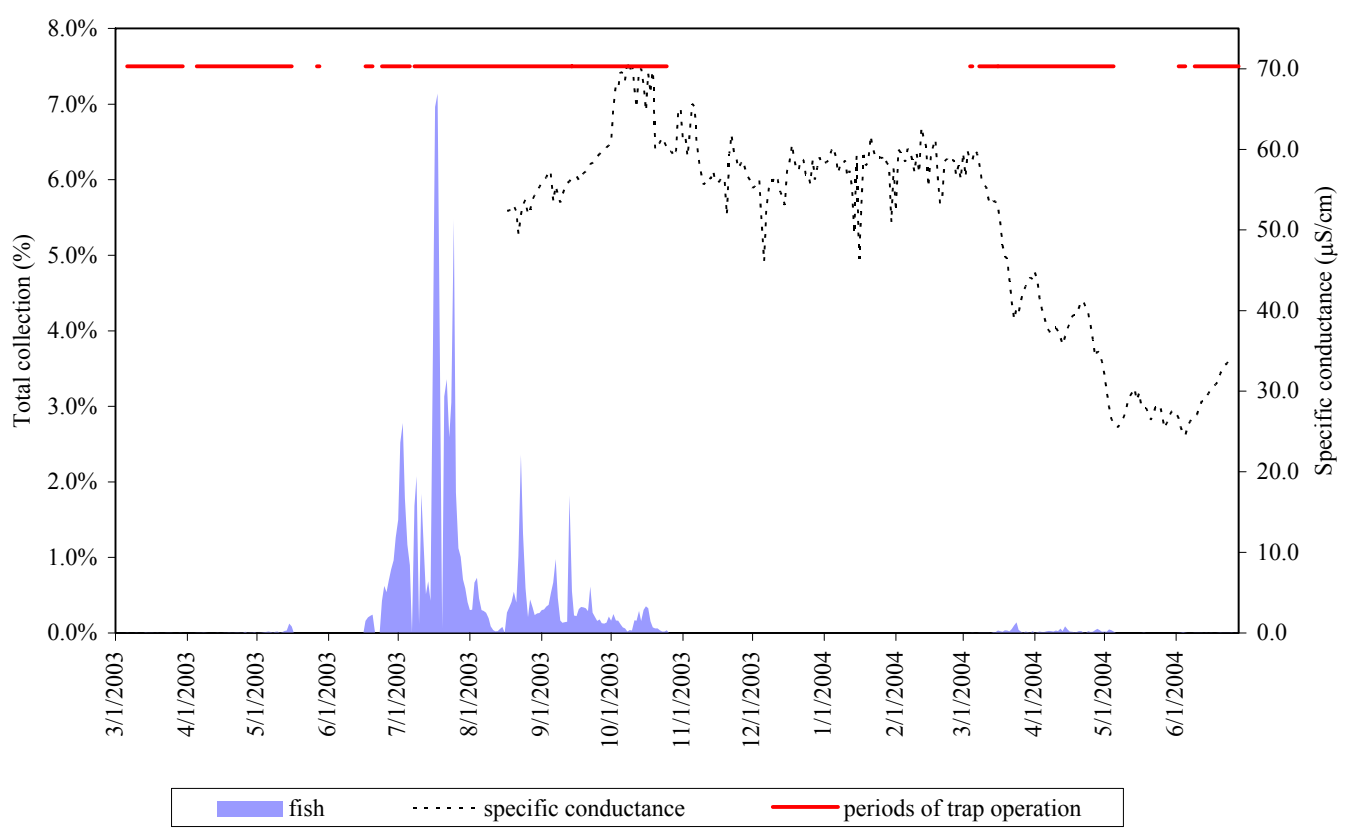

Secesh River Trap

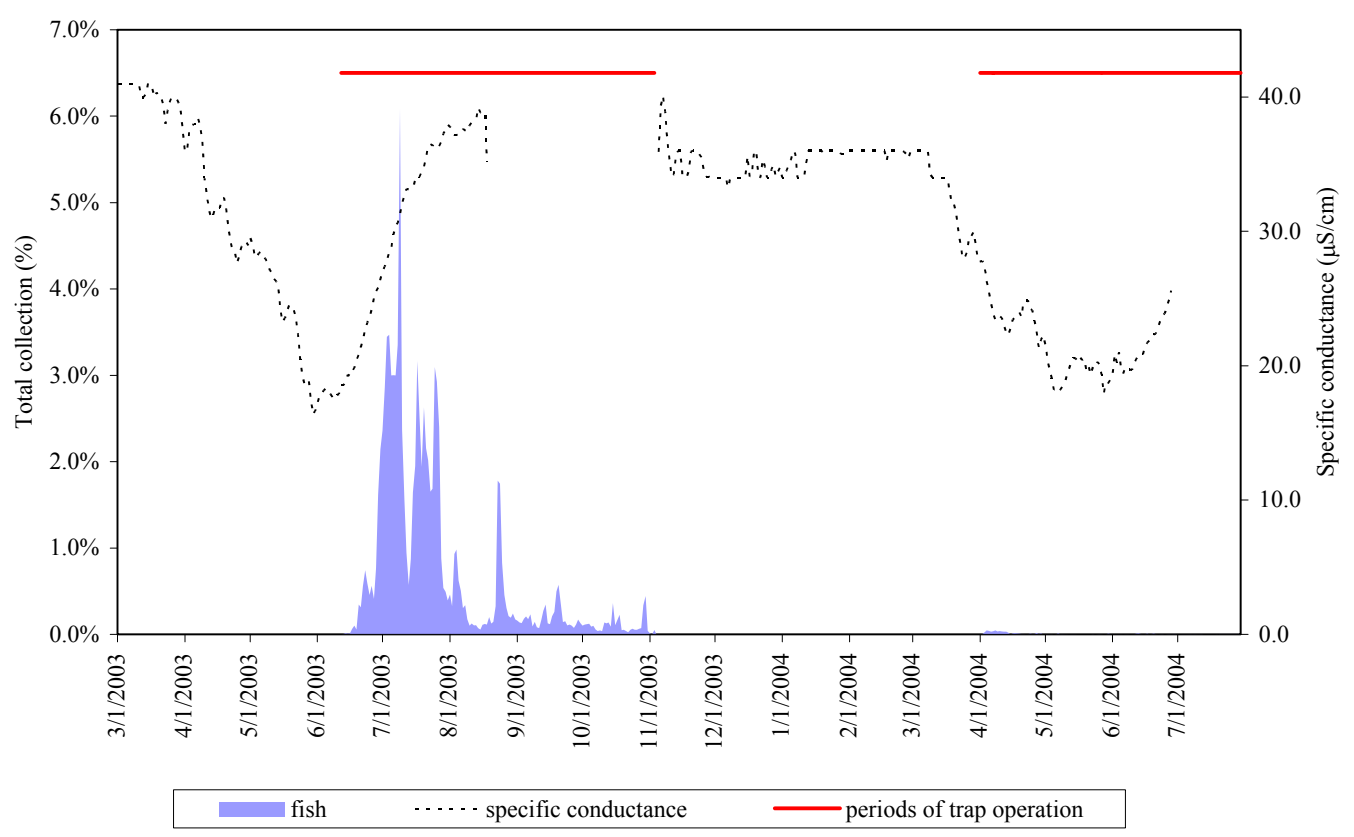

Appendix Figure 2. Continued. 
Upper Salmon River Trap
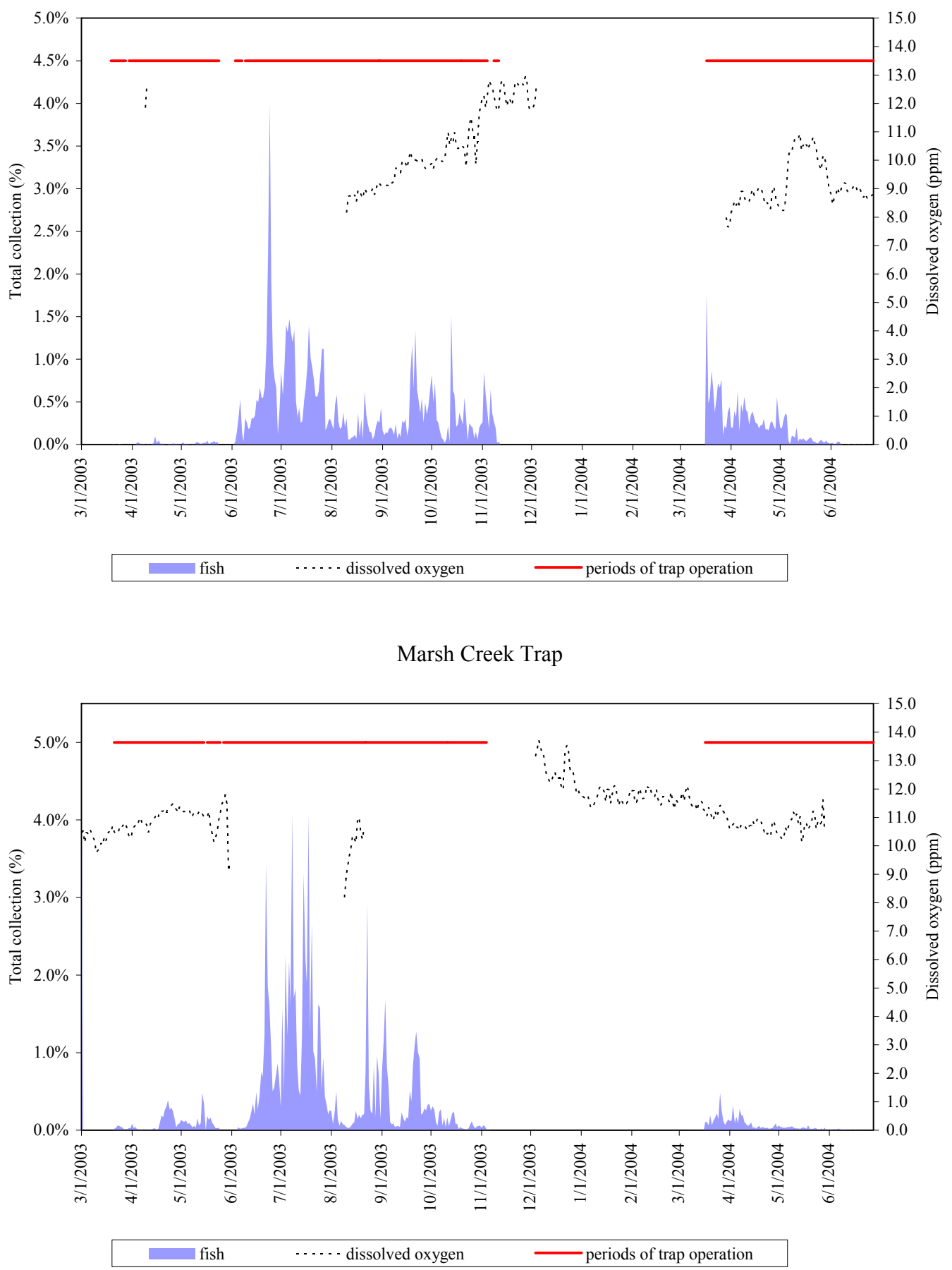

Appendix Figure 3. Daily passage of wild Chinook salmon fry, parr, and smolts at four migrant traps, expressed as percentages of total collected, and plotted against average daily dissolved oxygen collected near traps. Periods of trap operation are also shown. 
South Fork Salmon River Trap

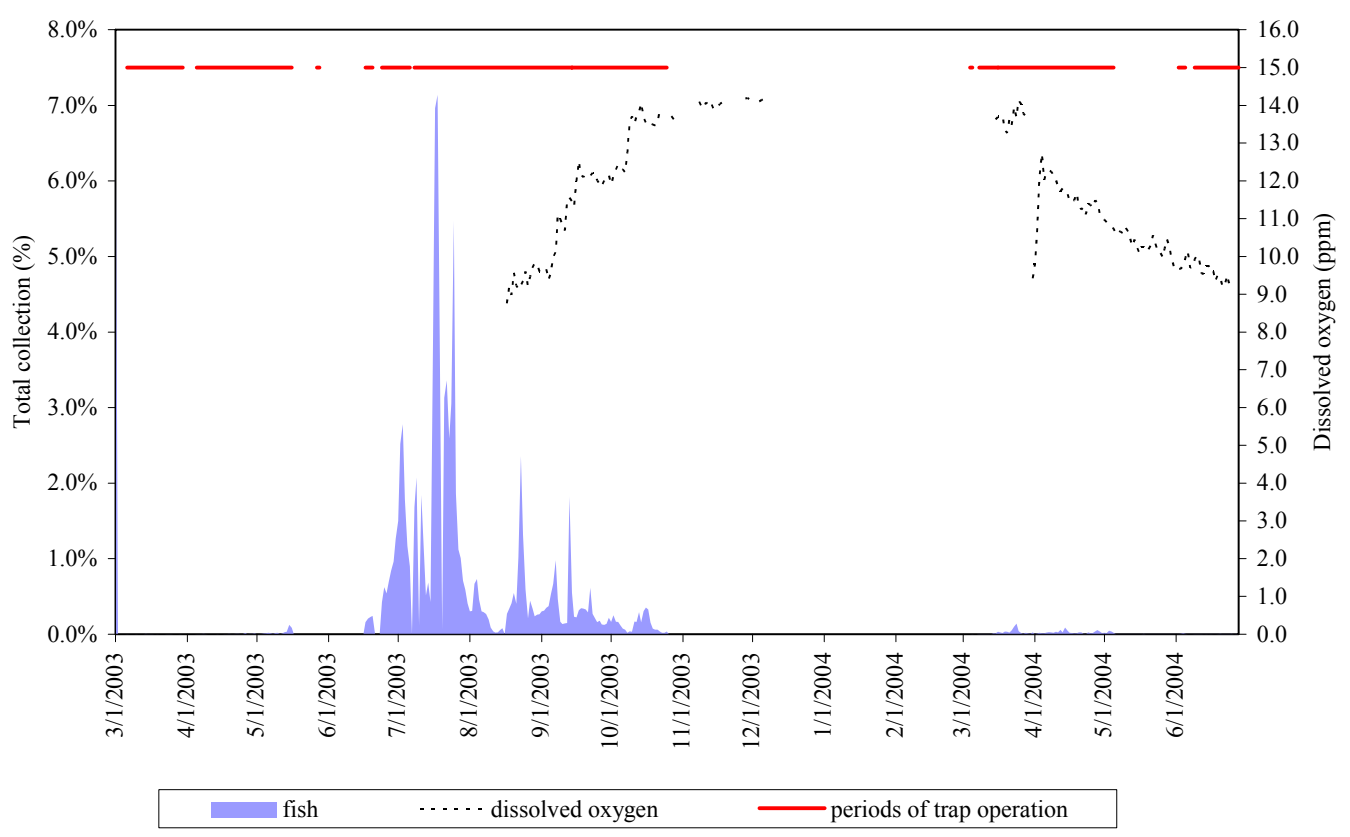

Secesh River Trap

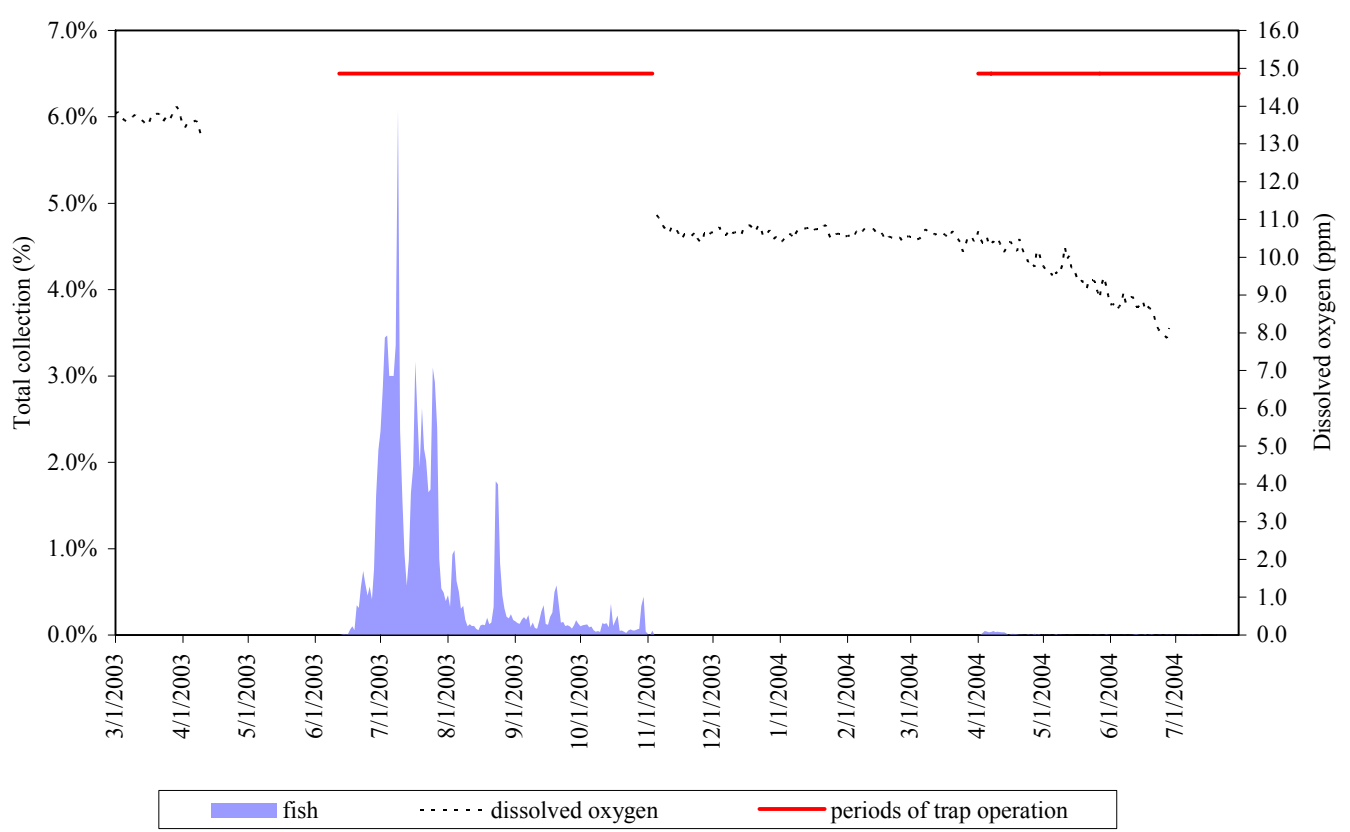

Appendix Figure 3. Continued. 
Upper Salmon River Trap

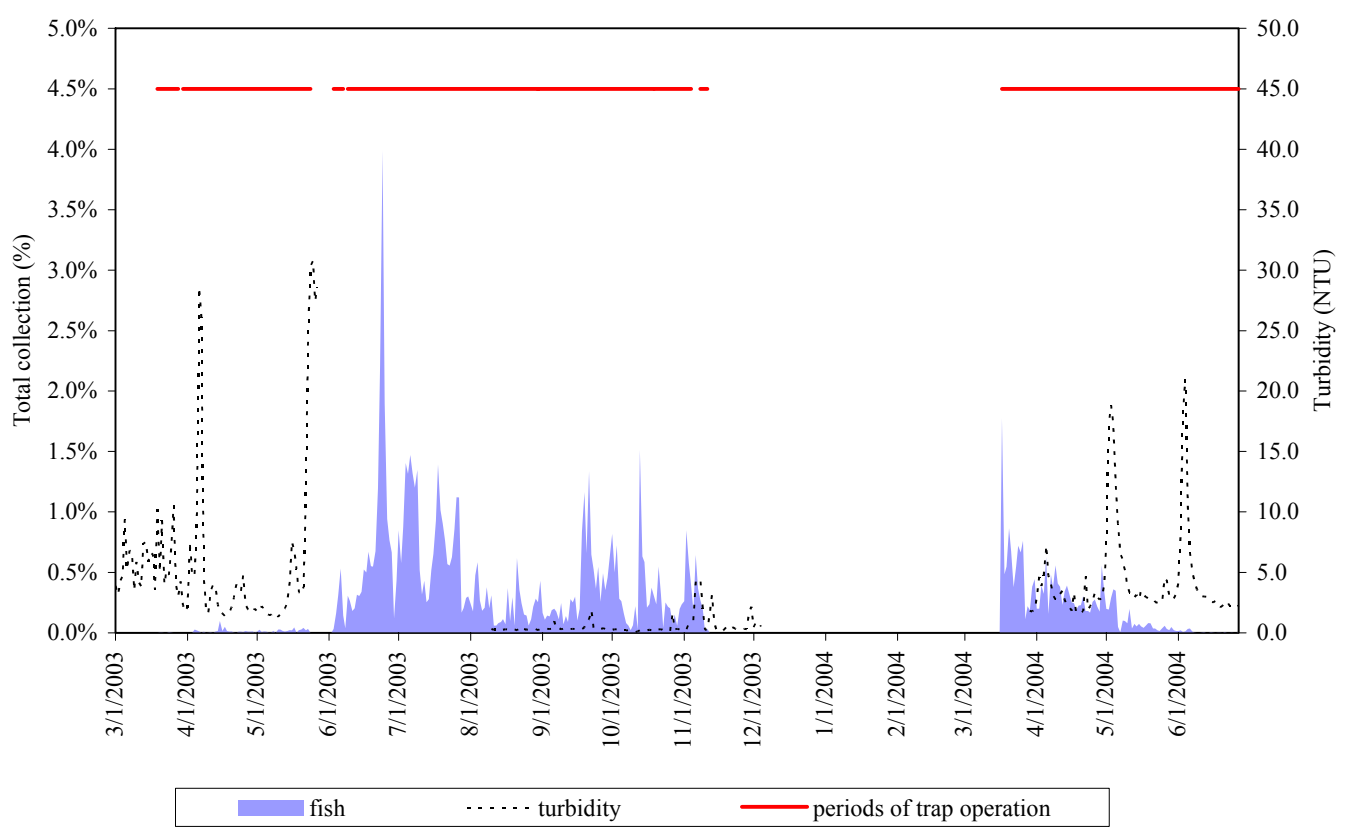

Marsh Creek Trap

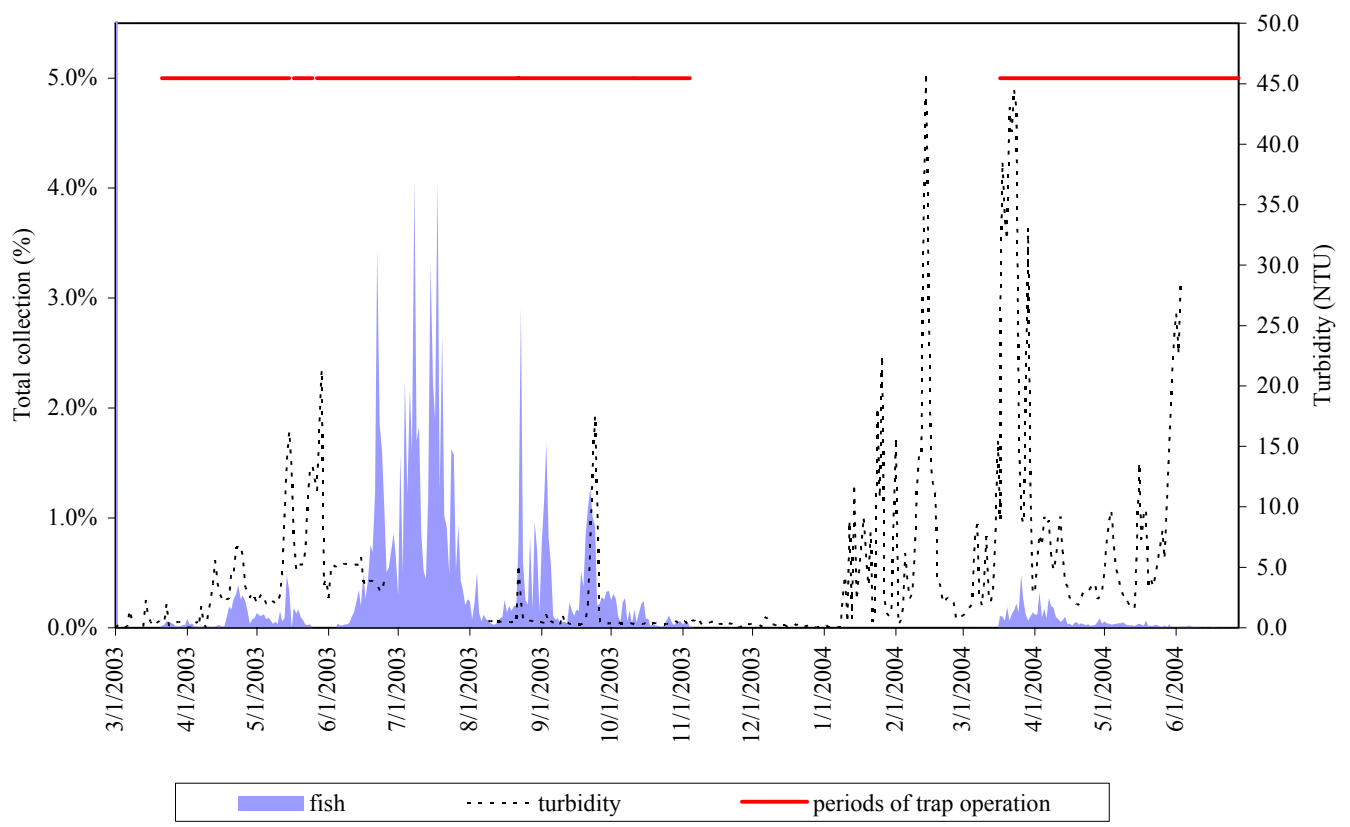

Appendix Figure 5. Daily passage of wild Chinook salmon fry, parr, and smolts at four migrant traps, expressed as percentages of total collected, and plotted against average daily turbidity collected near traps. Periods of trap operation are also shown. 
South Fork Salmon River Trap

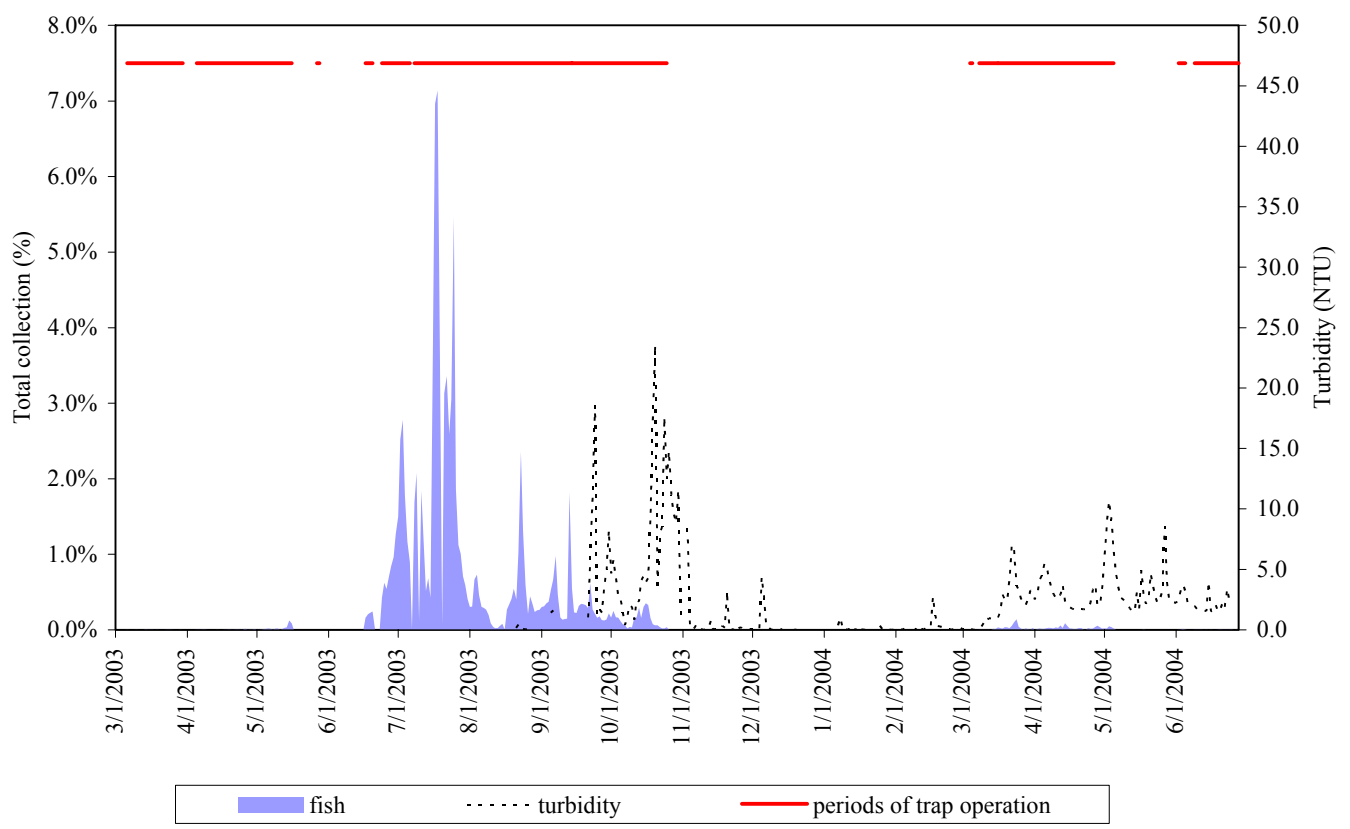

Secesh River Trap

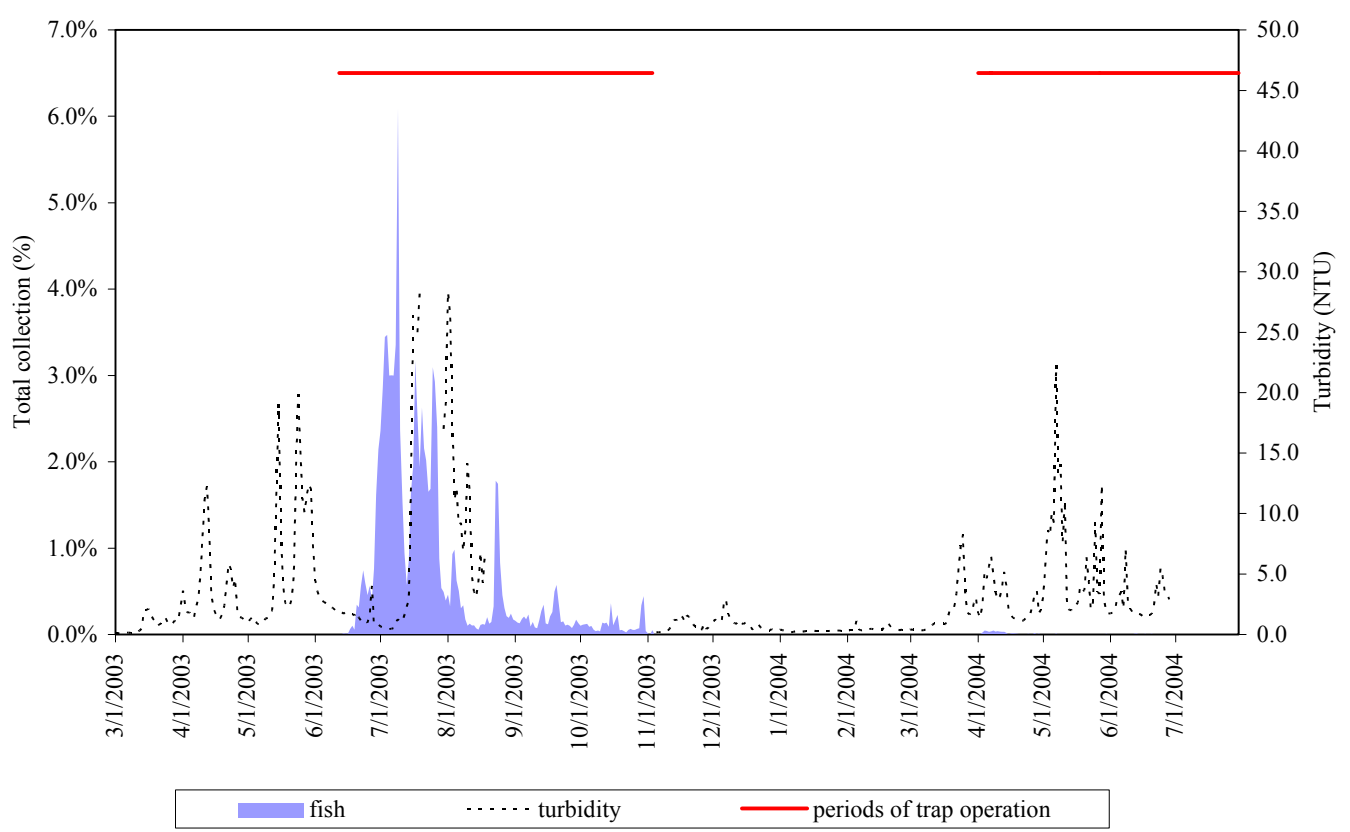

\section{Appendix Figure 5. Continued.}


Upper Salmon River Trap

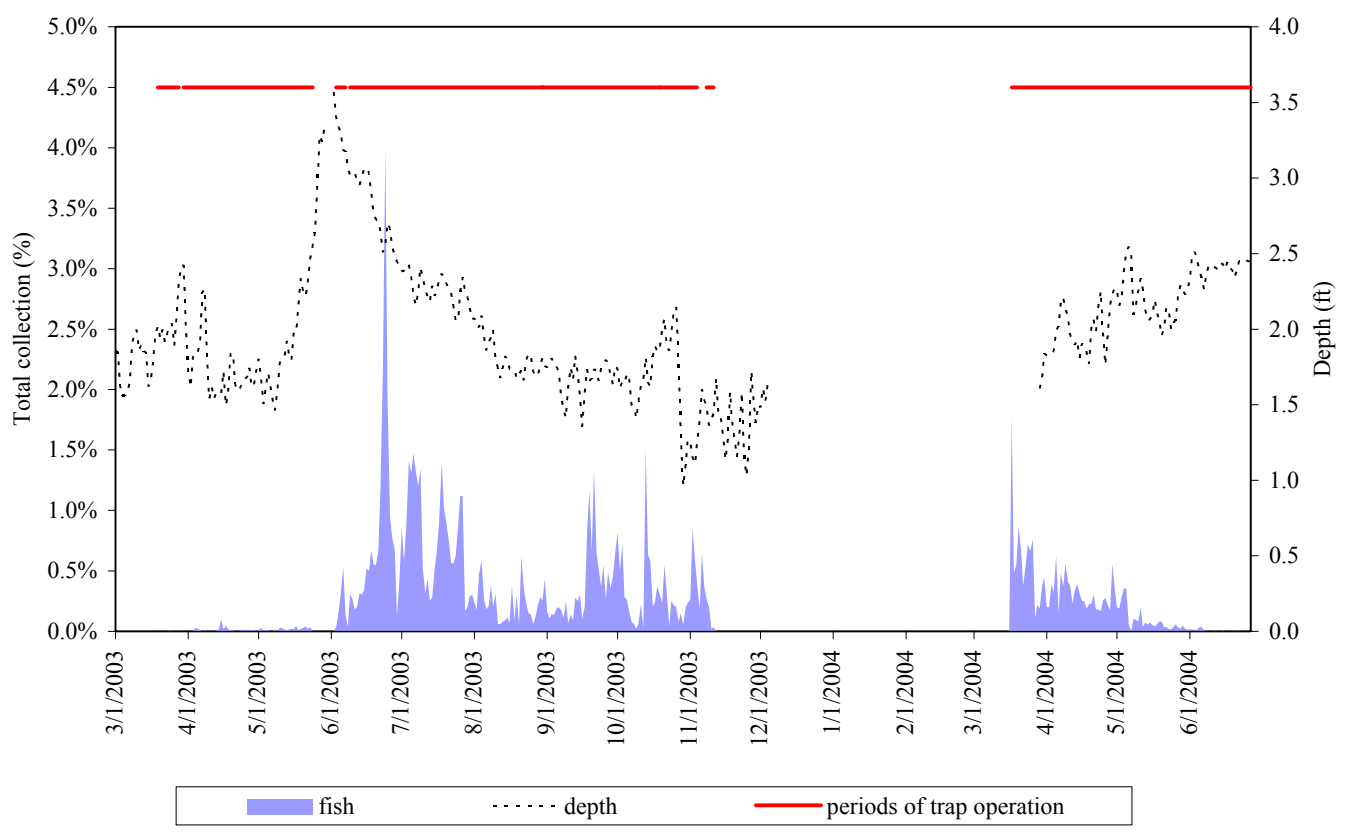

Marsh Creek Trap

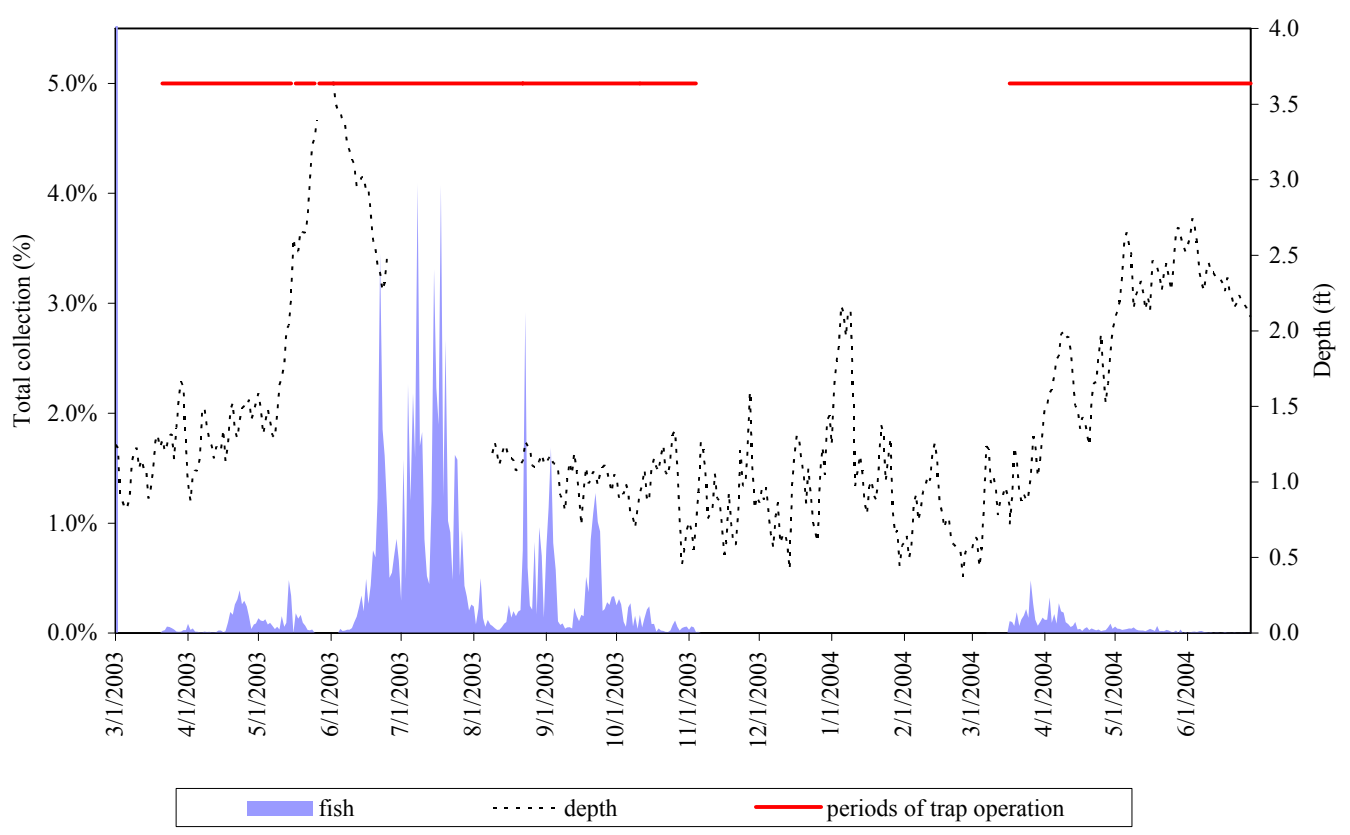

Appendix Figure 6. Daily passage of wild Chinook salmon fry, parr, and smolts at four migrant traps, expressed as percentages of total collected, and plotted against average daily depth collected near traps. Periods of trap operation are also shown. 


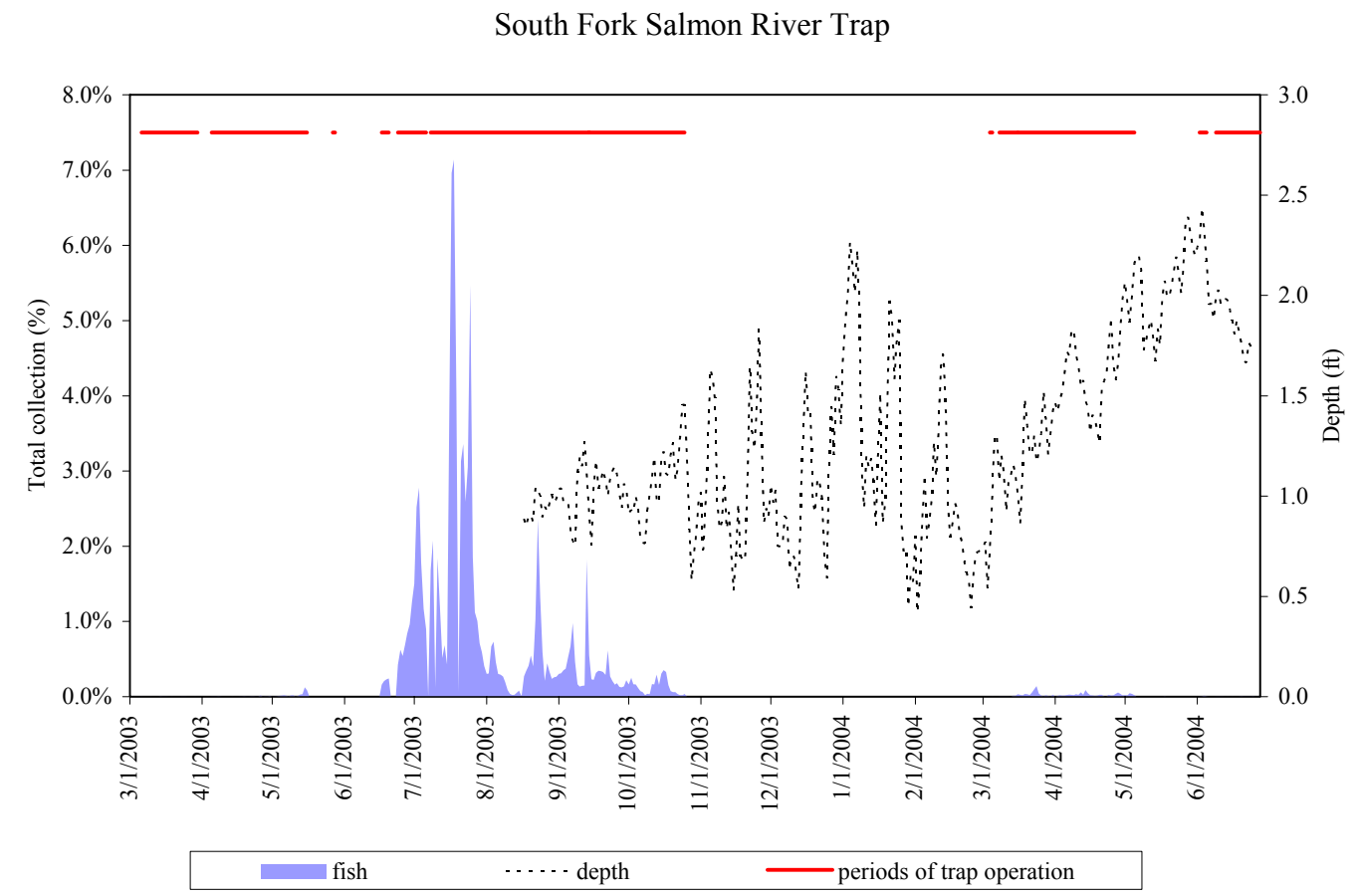

Secesh River Trap

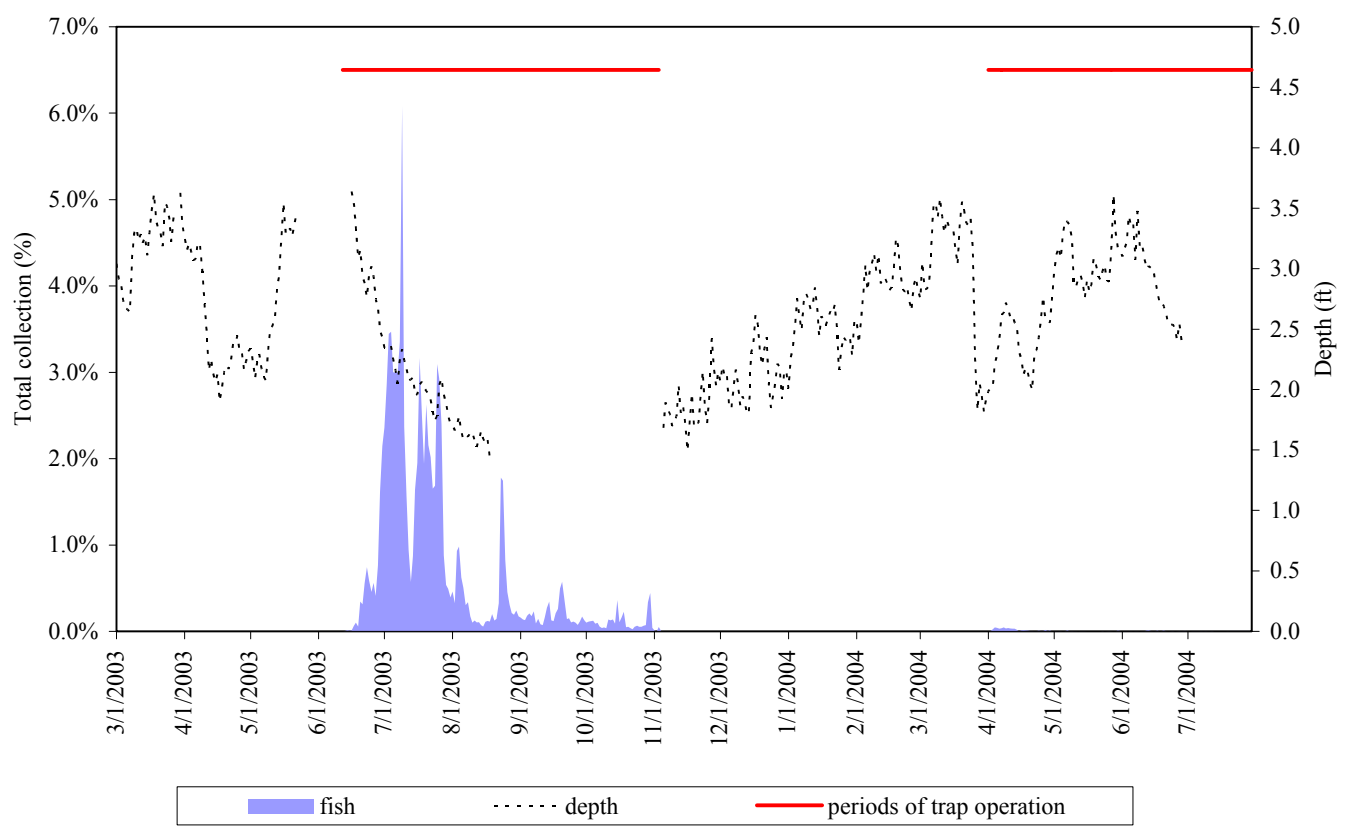

\section{Appendix Figure 6. Continued.}


Valley Creek PIT-tag Monitors

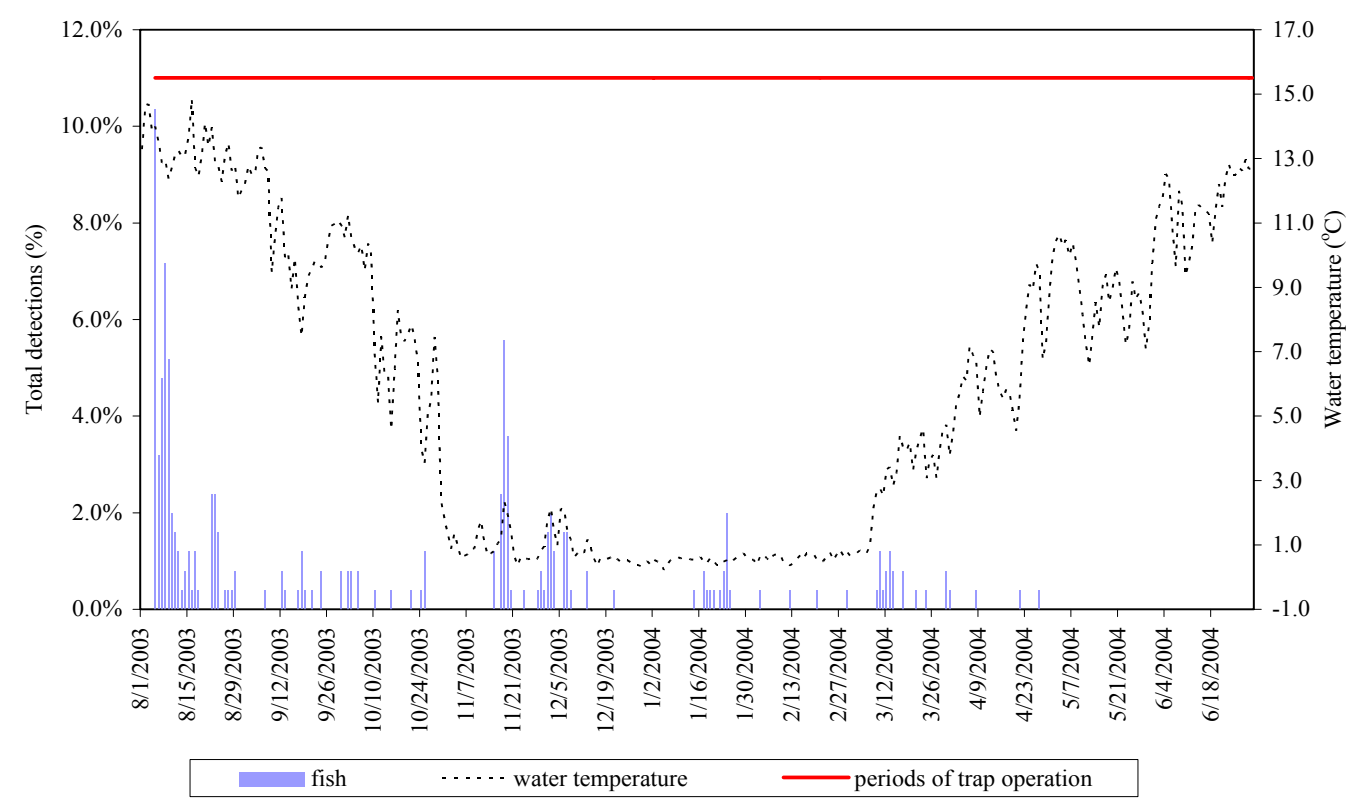

Valley Creek PIT-tag Detections

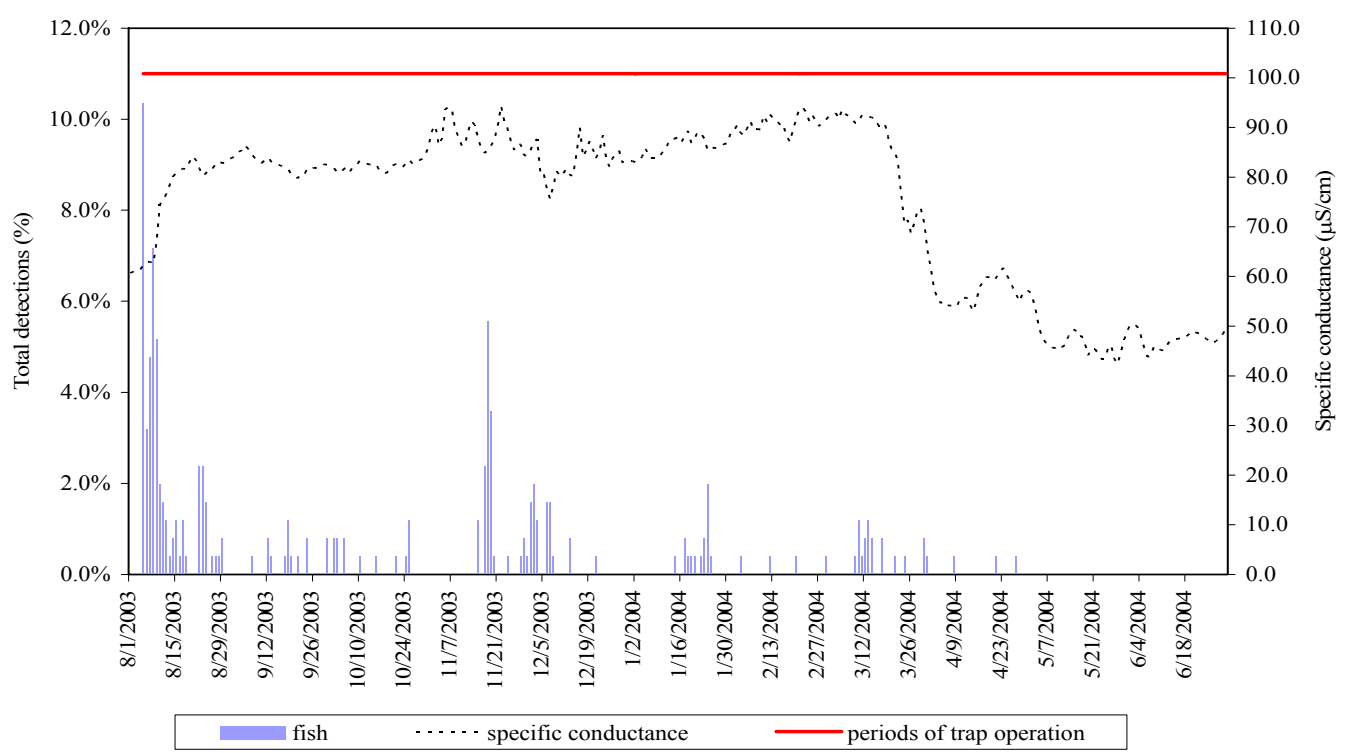

Appendix Figure 7. Combined daily PIT-tag detections of wild Chinook salmon parr at in-stream PIT-tag detectors in Valley Creek, expressed as percentages of total collected, and plotted against average daily aquatic conditions collected near the detectors. Periods of operation for the detectors are also shown. 


\section{Valley Creek PIT-tag Detections}

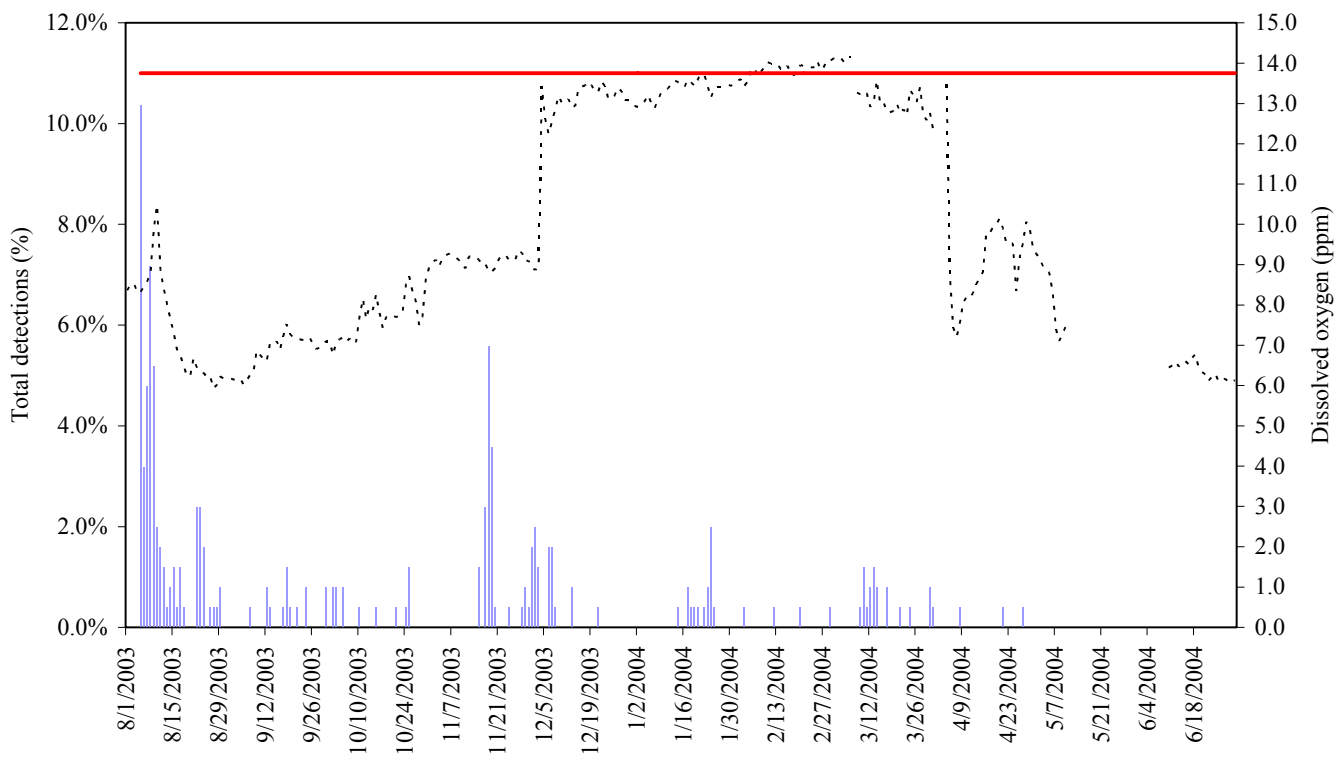

fish $\quad \cdots \cdot$. dissolved oxygen $\quad$ periods of trap operation

\section{Valley Creek PIT-tag Detections}

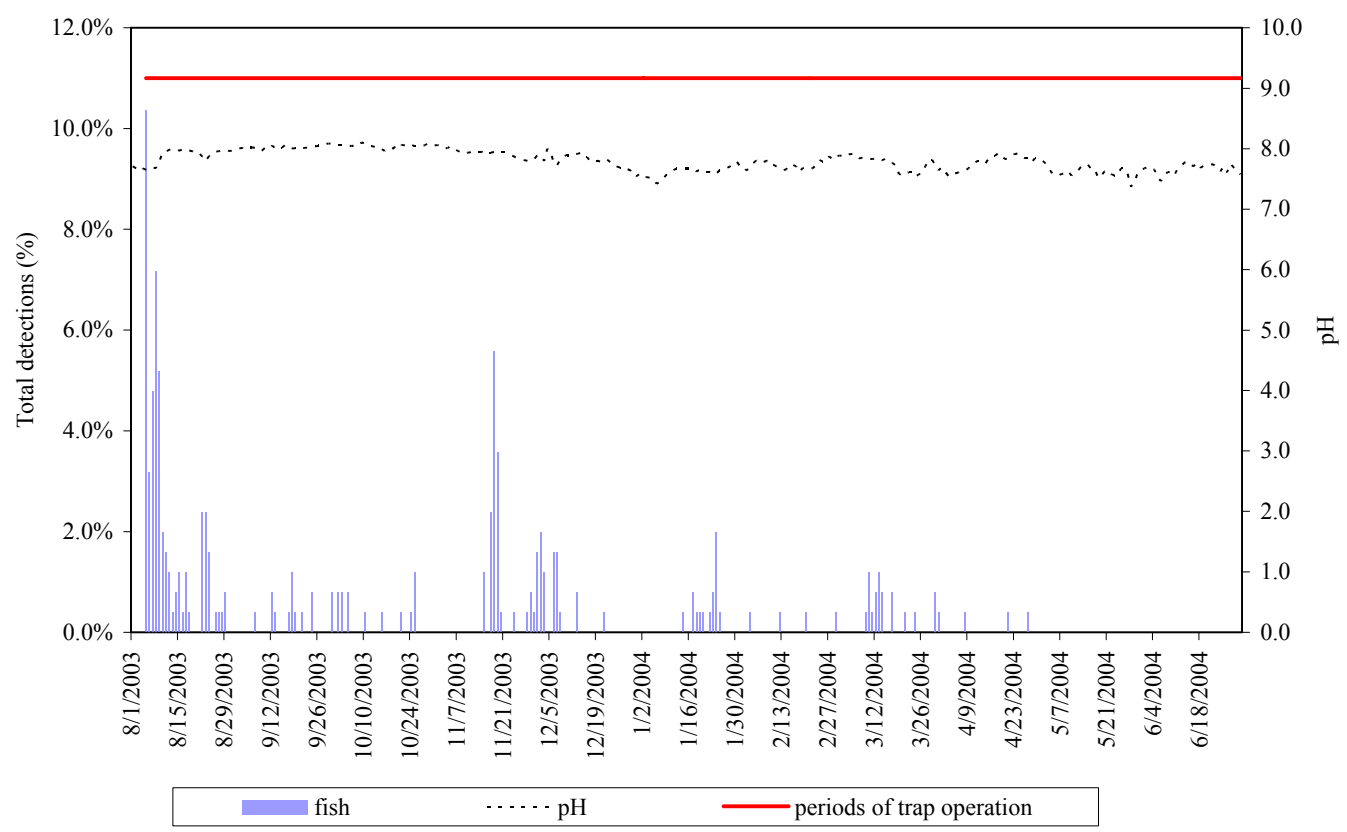

\section{Appendix Figure 7. Continued.}


Valley Creek PIT-tag Detections

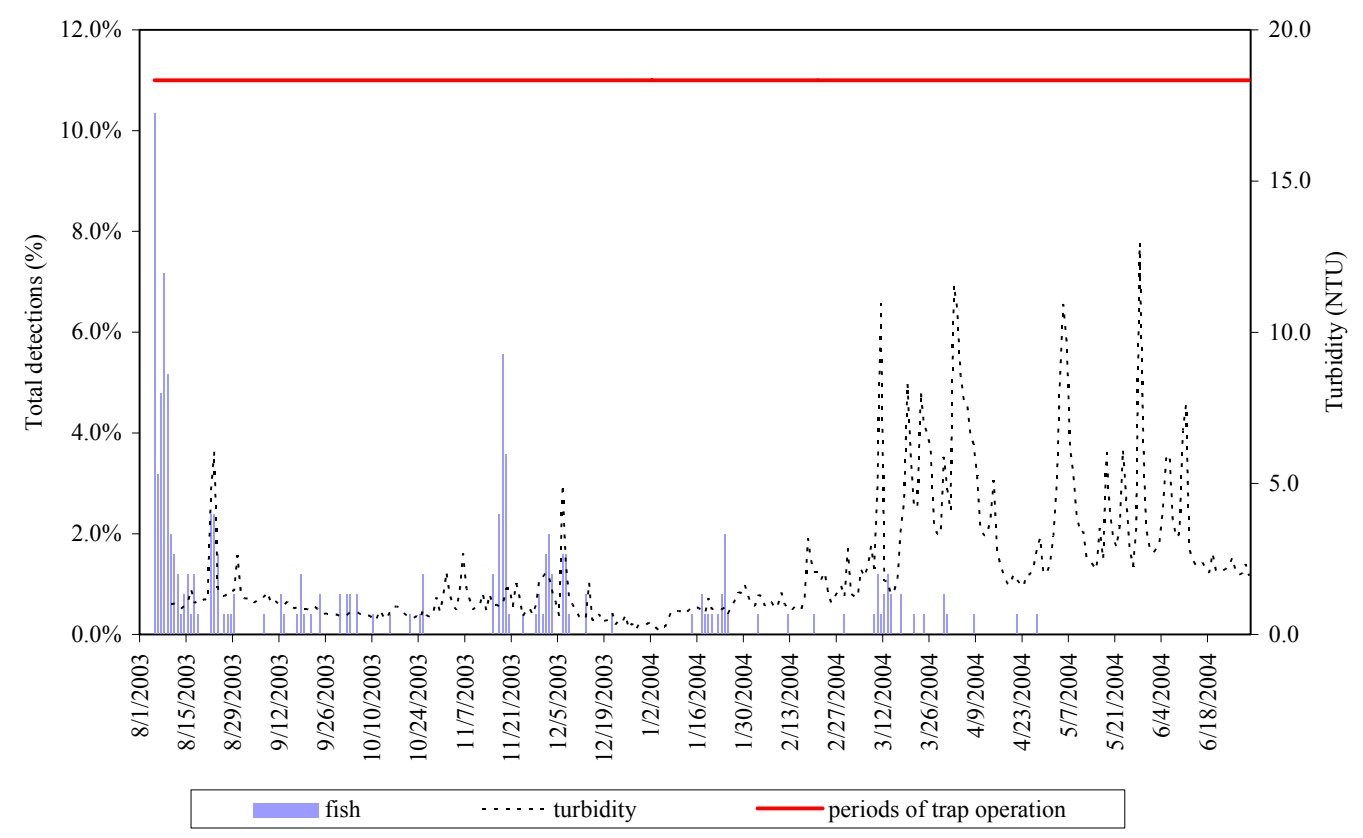

Valley Creek PIT-tag Detections

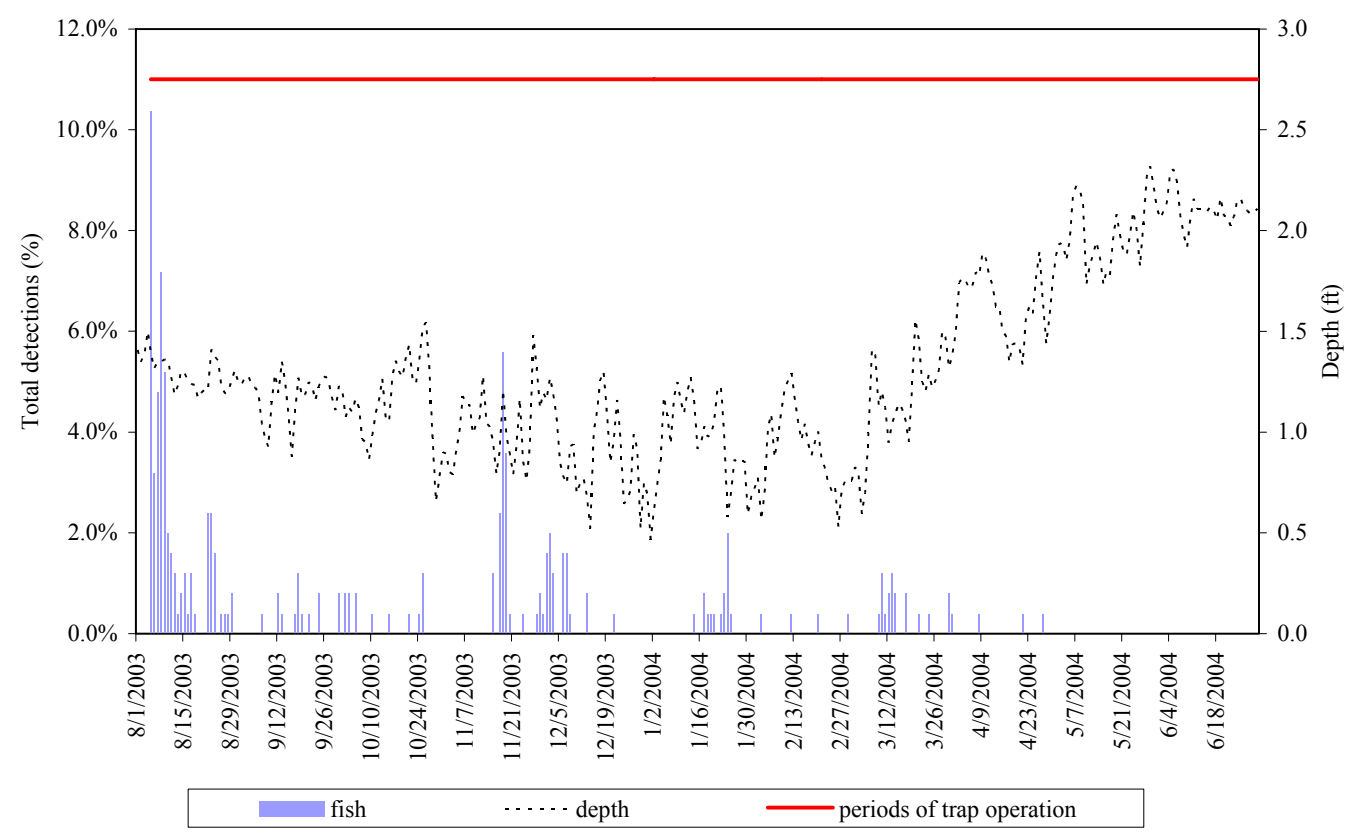

\section{Appendix Figure 7. Continued.}

